\title{
Photochemical Motion Control of Belousov-Zhabotinsky Droplets and Turbulence-like Patterns in the Aluminum Hydroxide Precipitation System
}

\author{
Syed Jazli Syed Jamaluddin \\ West Virginia University, ssyedjam@mix.wvu.edu
}

Follow this and additional works at: https://researchrepository.wvu.edu/etd

Part of the Physical Chemistry Commons

\footnotetext{
Recommended Citation

Syed Jamaluddin, Syed Jazli, "Photochemical Motion Control of Belousov-Zhabotinsky Droplets and Turbulence-like Patterns in the Aluminum Hydroxide Precipitation System" (2021). Graduate Theses, Dissertations, and Problem Reports. 8125.

https://researchrepository.wvu.edu/etd/8125

This Dissertation is protected by copyright and/or related rights. It has been brought to you by the The Research Repository @ WVU with permission from the rights-holder(s). You are free to use this Dissertation in any way that is permitted by the copyright and related rights legislation that applies to your use. For other uses you must obtain permission from the rights-holder(s) directly, unless additional rights are indicated by a Creative Commons license in the record and/ or on the work itself. This Dissertation has been accepted for inclusion in WVU Graduate Theses, Dissertations, and Problem Reports collection by an authorized administrator of The Research Repository @ WVU. For more information, please contact researchrepository@mail.wvu.edu.
} 
Photochemical Motion Control of Belousov-Zhabotinsky Droplets and Turbulence-like Patterns in the Aluminum Hydroxide Precipitation System

Syed Jazli Syed Jamaluddin

\author{
Dissertation submitted \\ to the Eberly College of Arts and Sciences \\ at West Virginia University
}

in partial fulfillment of the requirements for the degree of

Doctor of Philosophy in

Chemistry

\author{
Kenneth Showalter, Ph.D., Chair \\ Terry Gullion, Ph.D. \\ Adam Halasz, Ph.D. \\ Justin Legleiter, Ph.D. \\ Mark Tinsley, Ph.D. \\ C. Eugene Bennett Department of Chemistry \\ Morgantown, West Virginia \\ 2021
}

Keywords: Photosensitive BZ reaction, self-propelled particles, Marangoni flow, surface tension, precipitation-redissolution system, turbulence-like patterns, optical flow technique 


\begin{abstract}
Photochemical Motion Control of Belousov-Zhabotinsky Droplets and Turbulence-like Patterns in the Aluminum Hydroxide Precipitation System
\end{abstract}

Syed Jazli Syed Jamaluddin

A Belousov-Zhabotinsky (BZ) droplet that is immersed in an oil-phase can self-propel due to a surface tension gradient that exists on the droplet surface. The surface tension gradient is created as a result of the reactions that occur within and on the surface of the BZ droplet. By affecting the rates of these reactions, the BZ droplet direction can be controlled. In our experiments, we tune the reactions that occur within and on the BZ droplet surface by imposing a light intensity gradient on the droplet. We analyzed the BZ droplet motion and demonstrated that we can control the droplet directionality. We also demonstrated that the shape of the light intensity gradient imposed on the BZ droplet plays a role in controlling the directionality of the BZ droplet motion.

Turbulence-like patterns are characterized by chaotic dynamics in time and space. This behavior has been observed in unstirred BZ solutions. In this study, we show that turbulence-like patterns can form in the $\mathrm{AlCl}_{3} / \mathrm{NaOH}$ system. The patterns in this system form through precipitation and redissolution reactions. We utilized techniques such as the spatial correlation function, sink or source identification techniques, and the velocity angles statistics of the propagating precipitation waves to characterize the patterns formed in this system. 
To my late father, Syed Jamaluddin Syed Mohamed.

I will always miss you, abah.

$\sim$ Deli 


\section{Acknowledgment}

This piece of work has been a real labor and test of endurance, and I would have not reached this stage without the help and support from a lot of people. Therefore, there are a lot of people that I would like to say thank you to. First and foremost, I would like to thank my advisor, Dr. Kenneth Showalter, who has helped me a lot. His insight, suggestions, and constructive comments have helped me a lot in completing this work. I would also like to thank Dr. Mark Tinsley for the help he has provided especially when it comes to writing codes for my research projects. I would also like to say thank you to the rest of my graduate committee members: Dr. Terry Gullion, Dr. Adam Halasz, and Dr. Legleiter for reading and evaluating my dissertation.

Special thanks to Kritsana Khaothong from Kasetsart University, Bangkok, Thailand for helping me with my BZ droplet project. Not to forget the current and former members of the Showalter group: Dr. Darrell Collison, Dr. Desmond Yengi, David Mersing, Shannyn Tyler, Benjamas Ponboonjaroenchai, Md. Boshir Ahmed, and Kudakwashe Shumba. Thank you for all the help, suggestions, and ideas that you all have contributed.

Special thanks to my family: my mom and my sisters for constantly providing me with moral support and the encouragement throughout my graduate school program. Lastly to my closest friends: Renu, Maddie, and Rudy. Thank you for being there for me when I was at my lowest. Your presence has helped me march forward when I was going through a rough patch. 


\section{Contents}

Contents $\quad$ v

List of Figures viii

List of Tables $\quad$ xv

$\begin{array}{llr}1 & \text { Transport Phenomena in Chemical Systems } & 1\end{array}$

1.1 The Phenomenological Equations $\ldots \ldots \ldots \ldots$

1.2 Chemical Reactions and Transport Phenomena $\ldots \ldots \ldots$

1.3 References . . . . . . . . . . . . . . . . . . . . . . . . . . 11

\begin{tabular}{|lll}
\hline 2 & The Belousov-Zhabotinsky Reaction & 13
\end{tabular}

2.1 Introduction . . . . . . . . . . . . . . . . . . . . . . . . . . . 13

2.2 The Field-Körös-Noyes (FKN) Mechanism . . . . . . . . . . . . . . . . . 14

$2.3 \quad$ Self-organized Patterns in the Unstirred BZ Reaction $\ldots \ldots \ldots$. . . . . 17

$2.4 \quad$ Mathematical Modeling of the BZ reaction $\ldots \ldots \ldots \ldots$

$2.4 .1 \quad$ The Oregonator Model . . . . . . . . . . . . . . . . . . . . . . . . . . 19

2.4 .2 The Reaction-Diffusion Model . . . . . . . . . . . . . . . . . 20

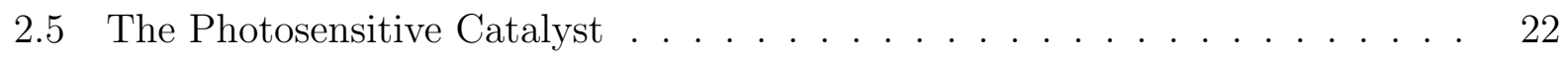

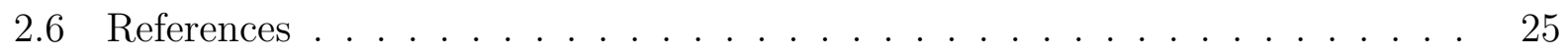


3 Belousov-Zhabotinsky Droplet Motion Control Using a Light Intensity

Gradient

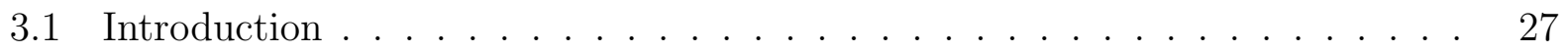

3.2 Experimental Method $\ldots \ldots \ldots \ldots$

3.3 Results . . . . . . . . . . . . . . . . . . . . . . . . . . . . . . . 30

$3.3 .1 \quad$ Homogeneous Light Intensity $\ldots \ldots \ldots$

$3.3 .2 \quad$ Constant Light Intensity Gradient . . . . . . . . . . . . . . . . . . . 32

$3.3 .3 \quad$ Complex BZ Droplet Trajectories $\ldots \ldots \ldots \ldots$

$3.3 .4 \quad$ BZ Droplet Trajectories as a Function of Time . . . . . . . . . . . . 38

3.4 Discussion $\ldots \ldots \ldots \ldots \ldots$

3.5 Conclusion . . . . . . . . . . . . . . . . . . . . . . . 46

3.6 References . . . . . . . . . . . . . . . . . . . . . . . . . . . . . . 48

4 Propagating Aluminum Hydroxide Precipitate under pH Field Influence 50

4.1 Introduction $\ldots \ldots \ldots \ldots \ldots \ldots$

4.2 Experimental Method $\ldots \ldots \ldots \ldots \ldots$

4.3 Results . . . . . . . . . . . . . . . . . . . . . . . . . . . . . . . 54

4.3 .1 Side-view Experiment $\ldots \ldots \ldots \ldots$

$4.3 .2 \quad$ Front-view Experiment $\ldots \ldots \ldots$

4.3 .3 Simulation . . . . . . . . . . . . . . . . . . . . . . . . . . . . . . . . 62

4.4 Discussion $\ldots \ldots \ldots \ldots$

4.5 Conclusion $\ldots \ldots \ldots \ldots$

4.6 References $\ldots \ldots \ldots \ldots \ldots$

5 Turbulence-like Patterns in the Aluminum Hydroxide System 73

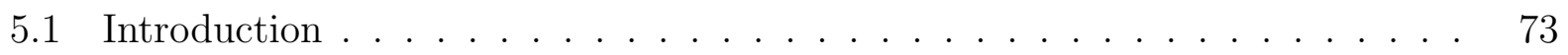




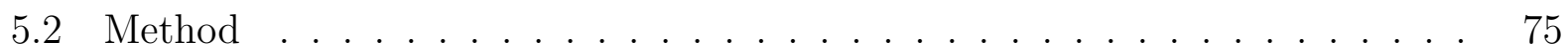

5.3 Results . . . . . . . . . . . . . . . . . . . . . . . . 76

5.3 .1 Image Acquisition and Velocity Determination . . . . . . . . . 76

5.3 .2 Source or Sink Identification Technique . . . . . . . . . . . . . 77

$5.3 .3 \quad$ Spatial Correlation Function $\ldots \ldots \ldots \ldots$

5.3 .4 Temporal Standard Deviation of the Velocity Angles _. . . . . . . . 81

5.4 Discussion $\ldots \ldots \ldots \ldots \ldots$

5.5 Conclusion $\ldots \ldots \ldots \ldots \ldots \ldots$

5.6 References $\ldots \ldots \ldots \ldots$ 


\section{List of Figures}

1.1 (a) The general definition of flux in a system. (b) The momentum flux in a fluid system. . . . . . . . . . . . . . . . . . . . . . . . 2

1.2 (a) A one-dimensional Liesegang band. The inner electrolyte is $\mathrm{Pb}\left(\mathrm{NO}_{3}\right)_{2}$ while the outer electrolyte is KI. Image taken from reference [4]. (b) A two-dimensional Liesegang pattern with $\mathrm{K}_{2} \mathrm{Cr}_{2} \mathrm{O}_{7}$ as the inner electrolyte and $\mathrm{AgNO}_{3}$ as the outer electrolyte. Image taken from reference [5]. (c) Cross-sectional experiment in $\mathrm{AlCl}_{3} / \mathrm{NaOH}$ system. Image taken from reference [12]. (d) A two-dimensional experiment of $\mathrm{AlCl}_{3} / \mathrm{NaOH}$ system showing a pair of counterrotating spiral waves. Image taken from reference $[15] . \ldots \ldots \ldots \ldots$

1.3 Aqueous Belousov-Zhabotinsky droplet in an oil-phase. The oil-phase is made up of squalane with surfactant (mono-olein) dissolved in it. The blue arrow indicates the direction that the droplet travels. . . . . . . . . . . . . . . . . . . . . . . . 9

$2.1 \quad$ A series of images of a stirred BZ solution. Images from Wikipedia. . . . . . . . 15 2.2 Plots of $\log _{10}$ (Concentration) as a function of time based on the Oregonator model for (a) bromous acid, (b) bromide ions, and (c) oxidized metal catalyst. The periodic sudden drop in bromide concentration represents the start of process B dominating the BZ reaction. . . . . . . . . . . . . . . . . . . . 21 
3.1 (a) The experimental setup used in controlling the BZ droplet motion in a monoolein/squalane mixture. (b) Chemical structure of the surfactant, monoolein.

(c) The trajectory angle $\theta$ definition. The angle $\theta$ range is $\left(-180^{\circ} \cup 180^{\circ}\right]$. Images

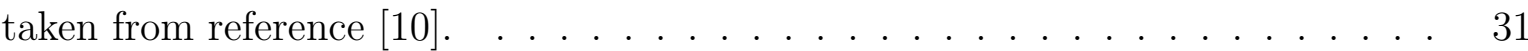

3.2 Results from the homogenous light intensity experiments. (a) Experiment with the reduced steady-state BZ droplet, $\left[\mathrm{Ru}(\mathrm{bpy})_{3}^{2+}\right]=1.88 \mathrm{mM}$, (i) droplet trajectory (ii) $\theta$-distribution (iii) droplet speed. (b) Experiment with the oxidized steady-state BZ droplet, $\left[\mathrm{Ru}^{2+}\right]=1.25 \mathrm{mM}$, (i) droplet trajectory (ii) $\theta$ distribution (iii) droplet speed. Other experimental conditions, $[\mathrm{MA}]=0.2 \mathrm{M},\left[\mathrm{NaBrO}_{3}\right]$ $=0.3 \mathrm{M},\left[\mathrm{H}_{2} \mathrm{SO}_{4}\right]=0.6 \mathrm{M},[\mathrm{NaBr}]=0.03 \mathrm{M}$, and [monoolein $]=10 \mathrm{mM}$. Image taken from reference $[10]$. . . . . . . . . . . . . . . . . . . . . . . . . . 33

3.3 Light intensity gradient applied on the BZ droplet in (a) constant light intensity gradient experiments and (b) complex BZ droplet trajectory experiments. The dashed line and the cross in (a) and (b) are considered the center of the control box for each case. The grayscale values and its corresponding light intensities are as follows: (a) $232=7.376 \times 10^{-4} \mathrm{~W} \mathrm{~cm}^{-2}$ and $140=2.383 \times 10^{-4} \mathrm{~W} \mathrm{~cm}^{-2}$, (b) $170=3.684 \times 10^{-4} \mathrm{~W} \mathrm{~cm}^{-2}$ and $60=4.632 \times 10^{-5} \mathrm{~W} \mathrm{~cm}^{-2}$. The background grayscale value and its corresponding light intensity value is $255=9.090 \times 10^{-4}$

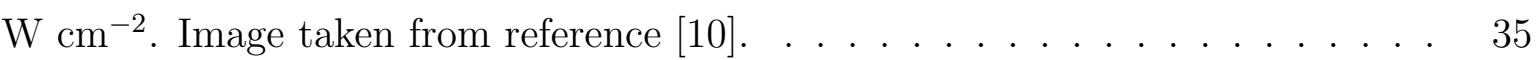

3.4 Results from one-dimensional BZ droplet direction control experiments.(a) Experiment with reduced steady-state BZ droplet, $\left[\mathrm{Ru}(\mathrm{bpy})_{3}^{2+}\right]=1.88 \mathrm{mM}$ (b) experiment with oxidized steady-state BZ droplet, $\left[\mathrm{Ru}^{2+}\right]=1.25 \mathrm{mM}$. Other experimental conditions, $[\mathrm{MA}]=0.2 \mathrm{M},\left[\mathrm{NaBrO}_{3}\right]=0.3 \mathrm{M},\left[\mathrm{H}_{2} \mathrm{SO}_{4}\right]=0.6 \mathrm{M},[\mathrm{NaBr}]$ $=0.03 \mathrm{M}$, and $[$ monoolein] $=10 \mathrm{mM}$. Image taken from reference $[10] . . . . .36$ 
3.5 Different BZ droplet trajectories that are produced by modifying the parametric equations 3.1 and 3.2 | (a) Circle, (b) 3-lobe hypotrochoid, (c) 4-lobe hypotrochoid, (d)-(f) modified hypotrochoids 1, 2, and 3, respectively. In each subplot, the actual trajectory followed by the droplet is shown, blue, along with the target trajectory, dotted red line, generated by the respective parametric equations. Immediately adjacent to each subplot is the angle $\theta$ distribution. For all experiments, the following BZ recipe is used: $\left[\mathrm{NaBrO}_{3}\right]=0.3 \mathrm{M},[\mathrm{MA}]=0.154 \mathrm{M}$, $[\mathrm{BrMA}]=0.046 \mathrm{M},\left[\mathrm{H}_{2} \mathrm{SO}_{4}\right]=0.6 \mathrm{M},[\mathrm{NaBr}]=0.03 \mathrm{M},\left[\mathrm{Ru}(\mathrm{bpy})_{3}^{2+}\right]=1.88 \mathrm{mM}$,

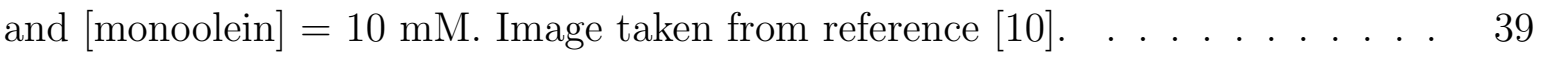

3.6 The BZ droplet trajectory for 3 laps of a 3-lobe hypotrochoid. (a) First, (b) second, and (c) third lap of the BZ droplet trajectory. The histogram to the right of each lap shows the corresponding angle $\theta$ distribution. Experimental conditions as in Figure 3.5 , Image taken from reference [10]. . . . . . . . . . . . 41

3.7 Simulation results using a modified Oregonator-type model. The reactions involved are listed in Table $3.1 \mid$ while the variables used are listed in Table 3.2 . (a) A plot of $\mathrm{Br}_{2}$ concentration as a function of dimensionless time for reduced (blue line) and oxidized (orange line) steady-state conditions. (b) A plot of light intensity as a function of dimensionless time. Images taken from reference [10]. .

4.1 Experimental setup used in this study. (a) The overall setup used in this study. The reactor used in the experiment is placed in between the camera and the light source. Unless noted, all of the experimental results presented here are backlit.

A schematic drawing of the reactor used for (b) side-view experiment and (c) front-view experiment. . . . . . . . . . . . . . . . . . . 55 
4.2 (a) A snapshot of the side-view experimental result. The red line indicates the slice used for constructing the spacetime plot. (b) A spacetime plot constructed from experiment in (a). The slope of the migrating band in the spacetime plot gives the speed of the migrating band: (i) $5.39 \times 10^{-4} \mathrm{~cm} \mathrm{~min}^{-1}$, (ii) $1.60 \times$ $10^{-5} \mathrm{~cm} \mathrm{~min}^{-1}$, and $1.18 \times 10^{-5} \mathrm{~cm} \mathrm{~min}^{-1}$. The experimental conditions are $\left[\mathrm{AlCl}_{3}\right]_{0}=0.16 \mathrm{M},[\mathrm{HCl}]_{0}=0.06 \mathrm{M},[\mathrm{NaOH}]_{0}=0.48 \mathrm{M}$, and $[$ agarose $]=1.0 \%$

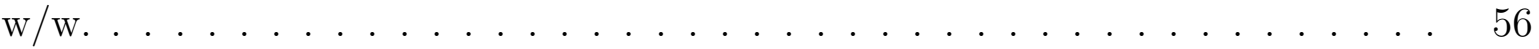

4.3 A plot of the migrating precipitation band position and its grayscale values as a function of time. The precipitation band migrates toward the $\mathrm{AlCl}_{3} / \mathrm{HCl}$ reservoir. The migration stops around 400 minutes after the band is detected. This is represented by the plateaued region around $0.5 \mathrm{~cm}$, which indicates that the band has stopped moving. While the band is migrating, the thickness of the band changes and this is shown by the oscillating grayscale values of the image. Experimental conditions are $\left[\mathrm{AlCl}_{3}\right]_{o}=0.08 \mathrm{M},[\mathrm{HCl}]_{o}=0.12 \mathrm{M},[\mathrm{NaOH}]_{o}=0.44$ $\mathrm{M}$, and $[$ agarose $]=1.0 \% \mathrm{w} / \mathrm{w} . \ldots \ldots \ldots \ldots$ 4.4 Migrating precipitation band speed as a function of (a) $\left[\mathrm{AlCl}_{3}\right]_{o}$ at $[\mathrm{HCl}]_{o}=0.12$ $\mathrm{M}$ and $[\mathrm{NaOH}]_{o}=0.48 \mathrm{M},(\mathrm{b})[\mathrm{HCl}]_{o}$ at $\left[\mathrm{AlCl}_{3}\right]=0.08 \mathrm{M}$ and $[\mathrm{NaOH}]_{o}=0.48 \mathrm{M}$, and $(\mathrm{c})[\mathrm{NaOH}]_{o}$ at $[\mathrm{HCl}]_{o}=0.012 \mathrm{M}$ and $\left[\mathrm{AlCl}_{3}\right]_{o}=0.08 \mathrm{M}$. The concentration of the agarose gel is $1.0 \% \mathrm{w} / \mathrm{w}$ in all of the experiments. . . . . . . . . . . . . . 59

4.5 A series of pictures showing the traveling precipitating wave fronts at $\left[A l C l_{3}\right]_{0}=$ $0.08 \mathrm{M},[\mathrm{HCl}]_{0}=0.12 \mathrm{M}$, and $[\mathrm{NaOH}]_{0}=0.48 \mathrm{M}$. Image (a) is taken 270 minutes after the experiment has started and the subsequent images are taken at 8 minutes apart. (b) Shows the waves annihilation as two wave fronts collide. . . 60 
4.6 A series of pictures showing the traveling precipitation wave fronts develop into spiral waves at $\left[\mathrm{AlCl}_{3}\right]_{0}=0.075 \mathrm{M},[\mathrm{HCl}]_{0}=0.11 \mathrm{M}$, and $[\mathrm{NaOH}]_{0}=0.41$ M. Image (a) is taken 170.5 minutes after the experiment has started, and the subsequent images are taken at 14 minutes apart. . . . . . . . . . . . . . . . . . 61

4.7 (a) A snapshot of the experiment result. The red line indicates the area used for constructing the spacetime plot. (b) A spacetime plot constructed from the experiment in (a). The slopes of the propagating waves in the spacetime plot give the speeds of the propagating waves. (i) $0.0107 \mathrm{~cm} \mathrm{~min}^{-1}$, (ii) $0.009 \mathrm{~cm} \mathrm{~min}^{-1}$, (iii) $0.0079 \mathrm{~cm} \mathrm{~min}^{-1}$, (iv) $0.0069 \mathrm{~cm} \mathrm{~min}^{-1}$, and (v) $0.0056 \mathrm{~cm} \mathrm{~min}^{-1}$. The experimental conditions are $\left[\mathrm{AlCl}_{3}\right]_{0}=0.02 \mathrm{M},[\mathrm{HCl}]_{0}=0.12 \mathrm{M},[\mathrm{NaOH}]_{0}=$ 0.44 M. . . . . . . . . . . . . . . . . . . . . 62

4.8 Plots showing the changes in the amount of the transmitted light in terms of grayscale values as a function of time for the front-view experiment. Higher grayscale values correspond to more transmitted light. (a) Change in grayscale value over the course of 11 hours of experiments. (b) Small amplitude oscillations that occur from 0 to $\sim 200$ minutes. The experimental conditions are $\left[\mathrm{AlCl}_{3}\right]_{0}=$

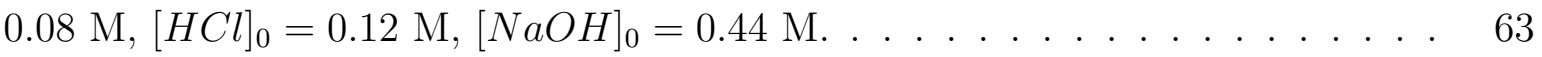
4.9 The effect of varying (a) $\left[\mathrm{AlCl}_{3}\right]_{o}$ at $[\mathrm{HCl}]_{o}=0.12 \mathrm{M}$ and $[\mathrm{NaOH}]_{o}=0.40 \mathrm{M}$, (b) $[\mathrm{HCl}]_{o}$ at $\left[\mathrm{AlCl}_{3}\right]_{o}=0.08 \mathrm{M}$ and $[\mathrm{NaOH}]_{o}=0.40 \mathrm{M}$, and $(\mathrm{c})[\mathrm{NaOH}]_{o}$ at $[\mathrm{HCl}]_{o}$ $=0.12 \mathrm{M}$ and $[\mathrm{HCl}]_{o}=0.12 \mathrm{M}$ on the speed of the propagating waves.....$\quad 63$

4.10 Results from simulation for the migrating band in this system. (a) The spacetime plot for the pH. (b) The spacetime plot from the simulation for the concentration of $\mathrm{AlCl}_{3}$ precipitate. . . . . . . . . . . . . . . . . . 66 
4.11 The effect of electrolytes concentration on the simulated migrating precipitation band. The conditions for the simulations are (a) (a) vary $\left[\mathrm{AlCl}_{3}\right]_{o}$ at $[\mathrm{HCl}]_{o}=$ $0.12 \mathrm{M}$ and $[\mathrm{NaOH}]_{o}=0.48 \mathrm{M},(\mathrm{b})$ vary $[\mathrm{HCl}]_{o}$ at $\left[\mathrm{AlCl}_{3}\right]_{o}=0.08 \mathrm{M}$ and $[\mathrm{NaOH}]_{o}$ $=0.48 \mathrm{M}$, and $(\mathrm{c})$ vary $[\mathrm{NaOH}]_{o}$ at $[\mathrm{HCl}]_{o}=0.12 \mathrm{M}$ and $\left[\mathrm{AlCl}_{3}\right]_{o}=0.08 \mathrm{M}$ on the speed of the migrating precipitation band. . . . . . . . . . . . . . . . 67

5.1 The experimental setup used to record the patterns formed in the $1 \%$ agarose gel. The recorded pattern images from this setup are used for the analysis to characterize the behavior of the waves that made up the patterns. . . . . . . . . 76

5.2 A series of images of the traveling waves that form in the agarose gel at various initial concentration of the aluminum chloride. $\left[\mathrm{AlCl}_{3}\right]_{0}:$ (a) $0.30 \mathrm{M}$, (b) $0.31 \mathrm{M}$, (c) $0.35 \mathrm{M},(\mathrm{d}) 0.40 \mathrm{M}$, (e) $0.43 \mathrm{M}$, and (f) $0.45 \mathrm{M}$. . . . . . . . . . . . . . 78 5.3 Velocity fields of the propagating precipitation waves at different initial concentrations of the aluminum chloride. $\left[\mathrm{AlCl}_{3}\right]_{0}:$ (a) $0.30 \mathrm{M}$, (b) $0.31 \mathrm{M}$, (c) $0.35 \mathrm{M}$, (d) $0.40 \mathrm{M}$, (e) $0.43 \mathrm{M}$, and (f) $0.45 \mathrm{M}$. The color represents the strength of the source or sink at different locations within the gel. . . . . . . . . . . . . . . . . . 79

5.4 Spatial correlation of the patterns formed in the agarose gel at different initial concentrations of the aluminum chloride. $\left[A l C l_{3}\right]_{0}:$ (a) $0.30 \mathrm{M}$, (b) $0.31 \mathrm{M}$, (c)

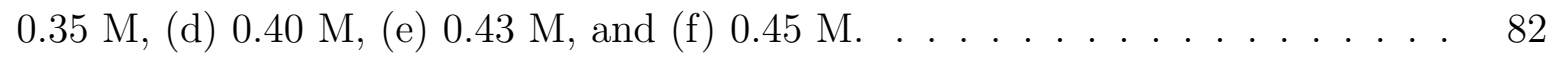
5.5 Temporal standard deviation of the propagating precipitation wave velocity angles. The colors for each panel correspond to the values of the temporal standard deviation in that region. The initial concentration of the aluminum chloride for each panel is (a) $0.30 \mathrm{M}$, (b) $0.31 \mathrm{M}$, (c) $0.35 \mathrm{M}$, (d) $0.40 \mathrm{M}$, (e) $0.43 \mathrm{M}$, and (f) $0.45 \mathrm{M} . \ldots \ldots \ldots . \ldots \ldots$ 
5.6 Plot of spatial means of temporal standard deviations for velocity angles as a function of initial aluminum chloride concentrations. The error bars represent the spatial standard deviations of the temporal standard deviations for the precipitate velocity angles. . . . . . . . . . . . . . . . . . . . . . . . . . 84 


\section{List of Tables}

3.1 Model variables $\mathrm{X}=\left[\mathrm{HBrO}_{2}\right], \mathrm{Y}=\left[\mathrm{Br}^{-}\right], \mathrm{Z}=\left[\mathrm{Ru}(\text { bpy })_{3}^{3+}\right], \mathrm{W}=\left[\mathrm{BrO}_{2} \cdot\right], \mathrm{V}=$ $\left[\mathrm{CHBr}(\mathrm{COOH})_{2}\right], \mathrm{P}=[\mathrm{HOBr}], \mathrm{E}=\left[\mathrm{Ru}(\mathrm{bpy})_{3}^{2+*}\right], \mathrm{G}=\left[\mathrm{Ru}(\mathrm{bpy})_{3}^{2+}\right]$ and $\mathrm{U}=\left[\mathrm{Br}_{2}\right]$. System parameters are given as $\mathrm{A}=\left[\mathrm{BrO}_{3}^{-}\right], \mathrm{H}=\left[\mathrm{H}^{+}\right]$, h is the stoichiometric factor, $\mathrm{C}_{0}$ is the total amount of ruthenium in the system, with $\mathrm{C}_{0} \approx \mathrm{Z}+\mathrm{G}$. $\mathrm{B}_{0}$ is a constant equal to the sum of the malonic acid and bromomalonic acid concentrations, with $\mathrm{B}=[\mathrm{MA}]=\mathrm{B}_{0}\left(1-\mathrm{V} / \mathrm{B}_{0}\right) . \ldots \ldots \ldots \ldots$

3.2 Rate constants and system parameters corresponding to the model given in Table

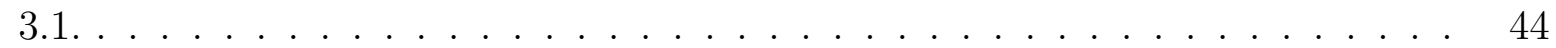




\section{Chapter 1}

\section{Transport Phenomena in Chemical}

\section{Systems}

Transport is the field of study that is concerned with the exchange of properties such matter, energy, and momentum of a system. Transport phenomena encompasses all of the physical changes that occur in nature. For an example, the energy exchange between the sun and the earth determines the weather patterns on earth. On a smaller scale, transport phenomena can be used to understand nutrient transport process within organisms such as the transport of sucrose from leaves to the roots of plants via phloem and the movement of water from the plant roots to the leaves via xylem. The vast applications make transport phenomena a very important field, and thus it has been studied by engineers, physicists, chemists, and biologists.

\subsection{The Phenomenological Equations}

Transport phenomena can be understood using a number of phenomenological equations, which are based on the concept of flux. Consider a system such as the one in Figure 
(a)

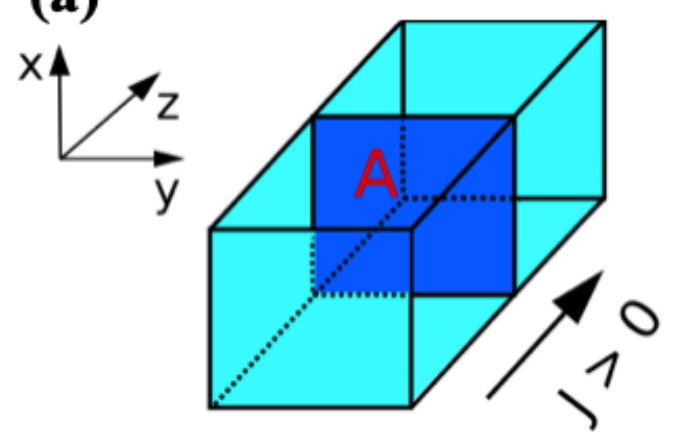

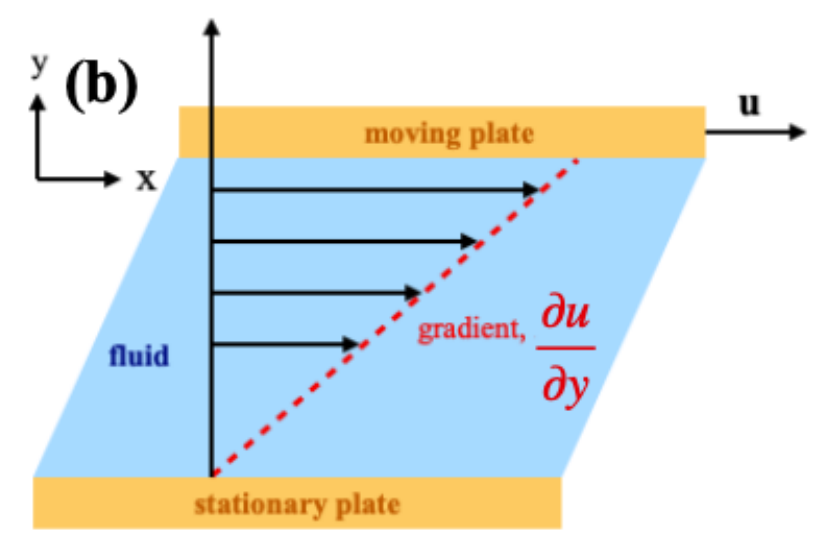

Figure 1.1: (a) The general definition of flux in a system. (b) The momentum flux in a fluid system.

1.1(a). The flux of property $p, J_{p}$, is the amount of property $p$ that passes through area $A$ in a given amount of time $t$ divided by $A$ and $t$. If the property of interest is matter flowing through the system, then we are speaking of a matter flux. According to Fick's first law of diffusion, the diffusive flux of species $i$ is directly proportional to the gradient of its concentration, and it can be written as follows:

$$
\begin{aligned}
J_{i} & \propto \frac{d C_{i}}{d z}, \\
& =-D_{i} \frac{d C_{i}}{d z},
\end{aligned}
$$

where $D_{i}$ is the diffusion coefficient of species $i$. The negative sign in equation 1.2 signifies that the species $i$ is moving down the concentration gradient that is from high concentration regions to low concentration regions. On the other hand, Fick's second law relates the change of concentration for species $i$ in time to the change of its concentration in space. Fick's second law can be written as follows:

$$
\frac{\partial C_{i}}{\partial t}=D_{i} \frac{\partial^{2} C_{i}}{\partial z^{2}}
$$

If a flux of energy such as heat is in consideration, then we are talking about a heat 
flux. A heat flux, $q$, is described by Fourier's law, and it can be written as follows:

$$
q=-k_{i} \frac{d T}{d z}
$$

where $k_{i}$ is the thermal conductivity of material $i$. Similar to the matter flux, the negative symbol in front of $k_{i}$ signifies that the heat flows from regions with high heat to regions with low heat, which is moving down the heat gradient.

The effect of momentum flux can be demonstrated by considering the system in

Figure 1.1(b), which shows a system where some fluid is sandwiched between two plates. The bottom plate is stationary while the top plate is moving with a constant velocity $u$. As the top plate moves, a thin layer of fluid next to the top plate moves with the same velocity as the top plate. The next layer of fluid also moves in the same direction as the first layer, but the velocity is less than $u$. Each subsequent layer of fluid will have a smaller velocity with the final layer that is next to the stationary plate has a velocity of zero. In this situation, there is a gradient of velocity $u$ with respect to direction $y$. The momentum flux can be written as follows:

$$
J=-\eta \frac{d u}{d y}
$$

where $\eta$ is the viscosity of the fluid. A fluid viscosity can be interpreted as the fluid resistance to deformation at a given rate. The resistance is attributed to the internal frictional force between the fluid layers.

\subsection{Chemical Reactions and Transport Phenomena}

Diffusion is driven by a concentration gradient, which causes a substance to spread out until it is distributed evenly in space. For example, if a drop of dye is added to a beaker of water, the dye will eventually spread and distribute throughout the entire beaker. If the 
diffusion process is coupled to chemical reactions, interesting phenomena can be observed. In the late 1800s, Liesegang reported that he obtained a series of concentric ring patterns in a gel by dissolving potassium chromate in the gel followed by placing a small drop of silver nitrate on top of the gel. The resulting concentric ring patterns have been called Liesegang patterns since its discovery.

A Liesegang system is a precipitation system whereby one of the electrolytes is immobilized in a gel matrix such as silica gel, polyvinyl alcohol, or gelatin. ${ }^{1-3}$ The immobilized electrolyte is called the inner electrolyte. A second electrolyte, called the outer electrolyte, is dissolved in water to make an aqueous solution of the electrolyte. The placement of the aqueous solution depends on the experimental setup used. For a one-dimensional experiment, the outer electrolyte is typically placed on top of the solid gel that contains the inner electrolyte. For a two-dimensional experimental setup, the center of the solid gel is typically removed, and the void is filled with the outer electrolyte.

The result of the experiment is dependent on the experimental setup used. In a one-dimensional system, the Liesegang pattern forms alternating bands that consist of precipitate and clear space, as shown in Figure 1.2(a). In a two-dimensional system, the Liesegang pattern forms concentric rings surrounding the outer electrolyte well. The concentric rings alternate between rings with the precipitate present and rings with clear space, as shown in Figure 1.2(b). There are several combinations of the inner electrolyte/outer electrolyte that can produce Liesegang patterns. Some examples of the inner electrolyte/outer electrolyte combination that have been investigated are $\mathrm{Pb}\left(\mathrm{NO}_{3}\right)_{2} / \mathrm{KI}, \mathrm{K}_{2} \mathrm{Cr}_{2} \mathrm{O}_{7} / \mathrm{AgNO}_{3}$, $\mathrm{Co}(\mathrm{OH})_{2} / \mathrm{NH}_{4} \mathrm{OH}, \mathrm{CaCl}_{2} /\left(\mathrm{NH}_{4}\right)_{2} \mathrm{CO}_{3}$, and $\mathrm{MgCl}_{2} / \mathrm{NH}_{4} \mathrm{OH} \cdot \underline{3}[\underline{6}$

Several theories have been proposed in order to explain the formation of Liesegang patterns, such as the supersaturation theory and the induced sol coagulation theory.1 According to the supersaturation theory, when the outer electrolyte diffuses into the gel, the 
(a)
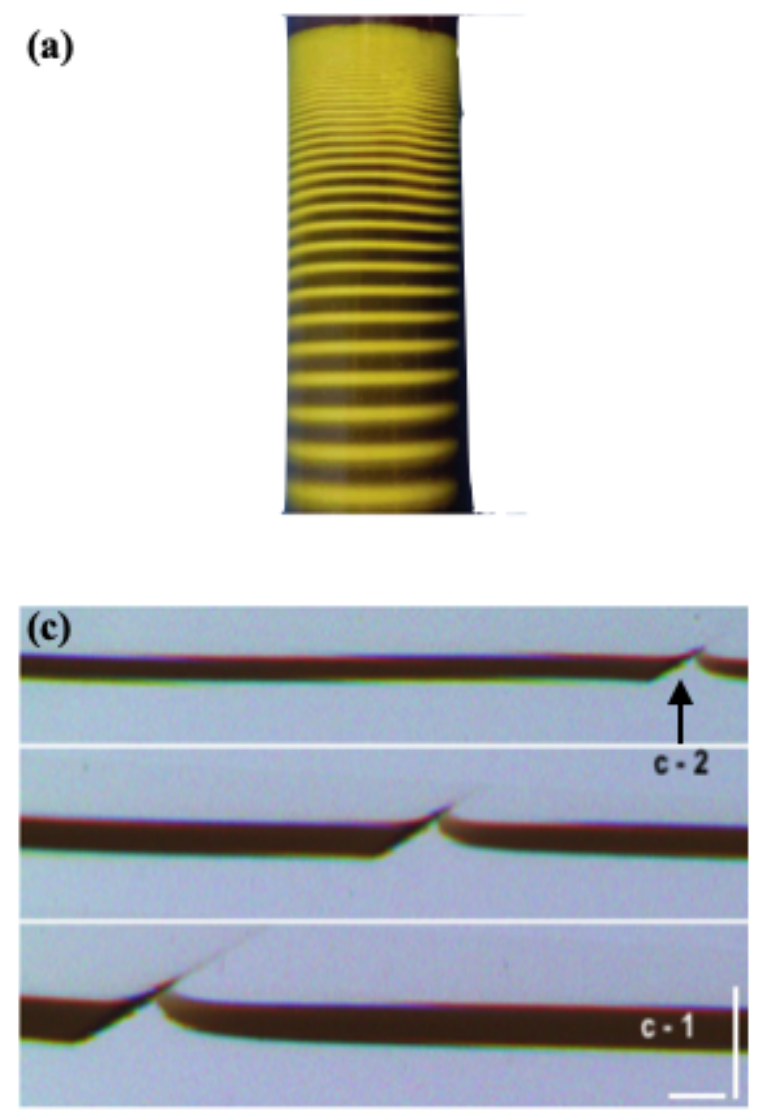

(b)

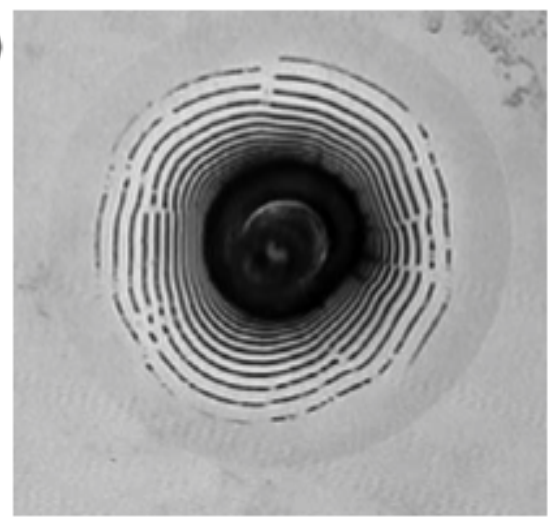

(d)

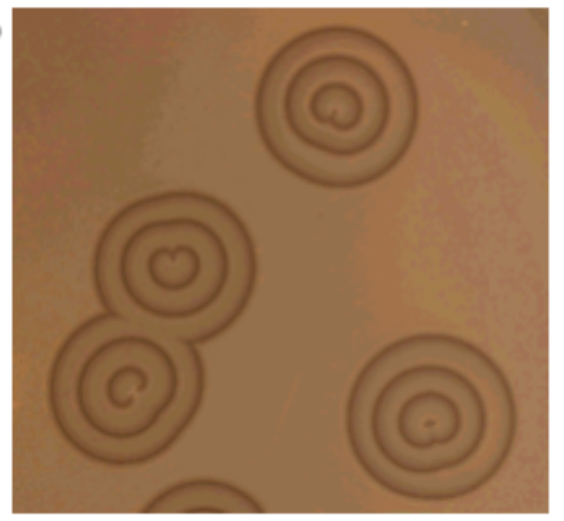

Figure 1.2: (a) A one-dimensional Liesegang band. The inner electrolyte is $\mathrm{Pb}\left(\mathrm{NO}_{3}\right)_{2}$ while the outer electrolyte is KI. Image taken from reference [4]. (b) A two-dimensional Liesegang pattern with $\mathrm{K}_{2} \mathrm{Cr}_{2} \mathrm{O}_{7}$ as the inner electrolyte and $\mathrm{AgNO}_{3}$ as the outer electrolyte. Image taken from reference [5]. (c) Cross-sectional experiment in $\mathrm{AlCl}_{3} / \mathrm{NaOH}$ system. Image taken from reference [12]. (d) A two-dimensional experiment of $\mathrm{AlCl}_{3} / \mathrm{NaOH}$ system showing a pair of counterrotating spiral waves. Image taken from reference [15].

electrolytes form a supersaturated solution within the gel. When the concentration of the supersaturated solution reaches its critical concentration, the precipitate starts to form. During the precipitation process, the electrolytes diffuse to the nucleation center. The electrolyte diffusion creates a region that is devoid of precipitate and electrolytes ahead of the precipitation layer. The outer electrolyte has to diffuse through this empty region before it encounters more of the inner electrolyte and repeats the cycle again. The repetition of the entire process creates the characteristic banding of the Liesegang patterns. Mathematically, the process is described by the coupling of the diffusion process and the precipitation reaction, and it can 
be written as follows:

$$
\begin{aligned}
\frac{\partial C_{a}}{\partial t} & =D_{a} \frac{\partial^{2} C_{a}}{\partial x^{2}}-\delta\left(C_{a}, C_{b}, K, L\right), \\
\frac{\partial C_{b}}{\partial t} & =D_{b} \frac{\partial^{2} C_{b}}{\partial x^{2}}-\delta\left(C_{a}, C_{b}, K, L\right), \\
\frac{\partial C_{p}}{\partial t} & =\delta\left(C_{a}, C_{b}, K, L\right),
\end{aligned}
$$

where $\mathrm{C}_{a}, \mathrm{C}_{b}$, and $\mathrm{C}_{p}$ are the concentration of the inner electrolyte, outer electrolyte, and precipitate, respectively. $\mathrm{D}_{a}$ and $\mathrm{D}_{b}$ are the diffusion coefficients of the inner electrolyte and outer electrolyte, respectively. The $\delta\left(C_{a}, C_{b}, K, L\right)$ is the precipitation reaction term, and it has the following definition:

$$
\delta(a, b, K, L)= \begin{cases}\kappa S_{p} \Theta\left(C_{a} C_{b}-K\right), & \text { if } C_{p}=0 \\ \kappa S_{p} \Theta\left(C_{a} C_{b}-L\right), & \text { if } C_{p}>0\end{cases}
$$

where $\kappa$ is the rate constant for the precipitation reaction, $L$ is the solubility product, $K$ is the nucleation product, and $\Theta$ is the Heaviside step function. $S_{p}$ is the amount of precipitate that can form, and it is defined as follows:

$$
S_{p}=\frac{C_{a}+C_{b}-\sqrt{\left(C_{a}+C_{b}\right)^{2}-4\left(C_{a} C_{b}-L\right)}}{2} .
$$

This model requires that the product of the electrolyte concentrations to reach the value $K$ before precipitation can occur. However, if there is some precipitate present at a location, the product of the electrolytes concentrations only has to reach a lower value $L$ to produce precipitate. $\frac{78}{78}$ The model indicates that the presence of precipitate in an area promotes the precipitation process.

On the other hand, the induced sol coagulation theory posits that after the diffusion of the outer electrolyte into the gel, the outer electrolyte immediately reacts with the inner electrolyte. The reaction produces a type of dispersed colloid called a sol that can diffuse within the gel. The sol is continuously produced and diffuses within the gel until it reaches its 
critical concentration. Once the critical concentration has been reached, the sol coagulates and precipitates out of the solution and forms a layer of precipitate. As the sol precipitates, it depletes the neighboring regions of the sol, thus creating a clear region with no precipitate and no inner electrolyte present. The outer electrolyte at the gel-solution interface has to diffuse further before it encounters more of the inner electrolyte, and the whole process starts again. The mathematical model of this system can be described by the following set of equations:

$$
\begin{aligned}
\frac{\partial C_{a}}{\partial t} & =D_{a} \frac{\partial^{2} C_{a}}{\partial x^{2}}-k_{1} C_{a} C_{b}, \\
\frac{\partial C_{b}}{\partial t} & =D_{b} \frac{\partial^{2} C_{b}}{\partial x^{2}}-k_{1} C_{a} C_{b}, \\
\frac{\partial C_{s}}{\partial t} & =D_{c} \frac{\partial^{2} C_{s}}{\partial x^{2}}+k_{1} C_{a} C_{b}-k_{2} C_{s} \Theta\left(C_{s}-C_{s}^{*}\right), \\
\frac{\partial C_{p}}{\partial t} & =k_{2} C_{s} \Theta\left(C_{s}-C_{s}^{*}\right),
\end{aligned}
$$

where $\mathrm{C}_{a}, \mathrm{C}_{b}, \mathrm{C}_{s}$, and $\mathrm{C}_{p}$ are the concentrations of the inner electrolyte, outer electrolyte, sol, and precipitate, respectively. $\mathrm{k}_{1}$ and $\mathrm{k}_{2}$ are the rate constants for the reaction of sol formation and precipitation reaction, respectively. $\mathrm{D}_{a}, \mathrm{D}_{b}$, and $\mathrm{D}_{s}$ are the diffusion coefficients of the inner electrolyte, outer electrolyte, and sol. $\Theta\left(C_{s}-C_{s}^{*}\right)$ is the Heaviside step function with $\mathrm{C}_{s}^{*}$ being the critical concentration at which the sol starts to precipitate out 19 11

Another type of system that is known to produce patterns via the coupling of diffusion processes and reaction is the precipitation-redissolution system. The experimental setup is similar to the Liesegang pattern experiment. The inner electrolyte is dissolved in a gel matrix such as agar, agarose, or gelatin while the outer electrolyte is dissolved in water and placed on top of the solid gel matrix containing the inner electrolyte. There are several combinations of inner electrolyte/outer electrolyte that have been studied in this system, which include $\mathrm{AlCl}_{3} / \mathrm{NaOH}, \mathrm{Al}(\mathrm{OH})_{4}^{-} / \mathrm{HCl}$, and $\mathrm{HgCl}_{2} / \mathrm{KI}$. 12

Experiments with the precipitation-redissolution system show that the patterns are 
made up of two main components. $\frac{15}{15}$ The first component is the migrating precipitation band that is initially formed at the gel-solution interface, and it travels down the solid gel matrix. The precipitate is continuously produced at the front of the migrating band while it is simultaneously redissolved at the back of the migrating band. The precipitation and redissolution process occurs because the molecules of the precipitate have the ability to form complex ions. For example, in the $\mathrm{AlCl}_{3} / \mathrm{NaOH}$ system, the following reactions occur:

$$
\begin{gathered}
\mathrm{Al}_{(a q)}^{3+}+3 \mathrm{OH}_{(a q)}^{-} \longrightarrow \mathrm{Al}(\mathrm{OH})_{3(s)}, \\
\mathrm{Al}(\mathrm{OH})_{3(s)}+\mathrm{OH}_{(a q)}^{-} \longrightarrow \mathrm{Al}(\mathrm{OH})_{4(a q)}^{-} .
\end{gathered}
$$

Equation 5.1 is the precipitation reaction while equation 5.2 is the complex-ion forming reaction that causes the redissolution. The second component of the precipitation-redissolution system is the formation of the self-organized pattern that propagates within the band. Figure 1.2 (c) shows the results of the $\mathrm{AlCl}_{3} / \mathrm{NaOH}$ cross-sectional experiment. The migrating precipitation band is labeled c-1, and the self-organized pattern that propagates within the band is labeled c-2 in Figure $1.2(\mathrm{c}) . \frac{12[13}{}$ Spiral patterns and target patterns have been observed in this system at lower concentrations $(₹ 0.32 \mathrm{M})$ of aluminum chloride while at higher concentrations $(\geqslant 0.35 \mathrm{M})$ of aluminum chloride the turbulence-like pattern is known to form.

The $\mathrm{AlCl}_{3} / \mathrm{NaOH}$ system can be mathematically modeled with a modified induced sol coagulation model. In this model, the inner electrolyte and outer electrolyte react and produce sol, which is converted into two different types of precipitate of $\mathrm{Al}(\mathrm{OH})_{3}$ called $\mathrm{p}_{1}$ and $\mathrm{p}_{2}$. In this model, $\mathrm{p}_{1}$ and $\mathrm{p}_{2}$ are hypothesized to have different crystal structures with $\mathrm{p}_{2}$ acting as a barrier to diffusion. $\mathrm{p}_{1}$ is hypothesized to form in the migrating band while $\mathrm{p}_{2}$ is hypothesized to form in the propagating wave within the migrating band. Specifically, $\mathrm{p}_{2}$ is generated at the wavefront of the propagating precipitation wave. $\frac{1213}{13}$ The conversion of sol 
to either precipitates only occurs if the concentration of sol is above its critical concentration. Additionally, the growth of $\mathrm{p}_{2}$ is further constrained to only occur if $p_{2}$ is already present at a location, or the neighboring location has the critical concentration for $\mathrm{p}_{2}$.

The viscosity of a fluid rarely plays an important role in chemical reactions. However, there is interesting chemistry that can be performed by utilizing viscosity especially if it is combined with surface tension. Surface tension can be interpreted as the energy needed to increase the area of a surface. The surface tension of a fluid can be lowered by introducing surfactants into the system. If the surface tension of a substance varies spatially, a surface tension gradient is created. The creation of the surface tension gradient on the surface is followed by the fluid flow from low surface tension regions to high surface tension regions to offset the tangential stress created by the surface tension gradient. The fluid flow caused by the gradient of surface tension is called Marangoni flow.

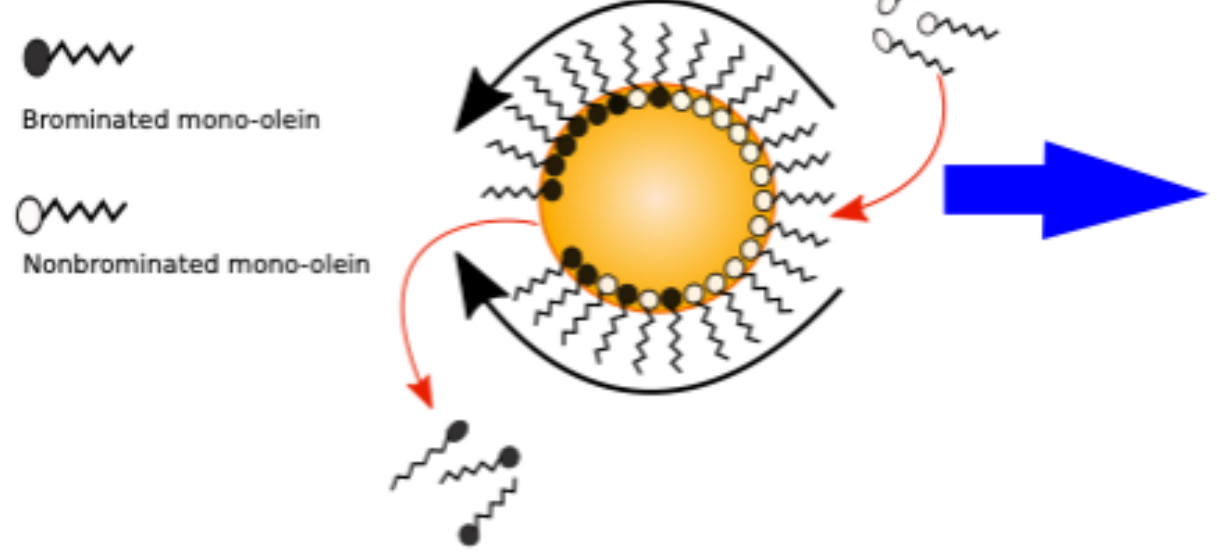

Figure 1.3: Aqueous Belousov-Zhabotinsky droplet in an oil-phase. The oil-phase is made up of squalane with surfactant (mono-olein) dissolved in it. The blue arrow indicates the direction that the droplet travels.

Scientists have utilized the Marangoni flow to do interesting chemistry, such as the production of various systems of self-propelled particles. One of the systems of selfpropelled particles involves the production of an aqueous Belousov-Zhabotinsky (BZ) droplet 
in an oil-phase that contains a surfactant. The surfactant lowers the surface tension of the droplet. However, the BZ reaction that occurs in the droplet continuously produces bromine molecules. The bromine molecules react with some of the surfactant on the droplet surface. Consequently, a surface tension gradient is created on the surface of the BZ droplet. Since the gradient of the surface tension has to be offset by the tangential stress on the surface, there is a flow of fluid from the low surface tension regions to high surface tension regions. Due to the viscosity of the the oil-phase, there is a momentum transfer between the surface of the droplet to the oil-phase, which results of the flow in the surrounding fluid. The momentum transfer helps to propel the droplet forward $\stackrel{16117}{ }$ Figure 1.3 shows a schematic drawing of the propulsion mechanism for the BZ droplet propulsion. The dependence on the Marangoni flow to propel particles forward has been applied to other systems such as a camphor boat on a water surface, oleic acid droplet on an aqueous phase of azobenzene trimethylammonium bromide (AzoTAB), and water droplets in squalane ${ }^{\sqrt{18 \mid 20}}$ The movement of the self-propelled particles can be controlled by affecting the surfactant property. In the case of the BZ droplet, a light intensity gradient has been used to change the property of the surfactant via $\mathrm{BZ}$ reaction kinetics manipulation. ${ }^{[2122}$ In the case of the oleic acid on aqueous AzoTAB, light with different wavelengths has been used to affect the molecular structure of AzoTAB, the surfactant, to control the movement of the oleic acid droplet.19 


\subsection{References}

[1] K. H. Stern, Chem. Rev. 54, 79 (1954).

[2] M. Matsue, M. Itatani, Q. Fang, Y. Shimizu, K. Unoura, and H. Nabika, Langmuir 34, 11188 (2018).

[3] K. M. Pillai, V. K. Vaidyan, and M. A. Ittyachan, Colloid and Polymer Science 258, 831 (1980).

[4] A. Toramaru and T. Harada and T. Okamaru, Phys. D 183, 133 (2003).

[5] I. Lagzi, A. Volford, and A. Büki, Chem. Phys. Lett. 396, 97 (2004).

[6] H. Batlouni and M. Al-Ghoul, J. Phys. Chem. A 112, 8038 (2008).

[7] F. Izsák and I. Lagzi, J. Chem. Phys. 120, 1837 (2004).

[8] A. Büki, É. Kárpáti-Smidrózki, and M. Zrínyi, J. Chem. Phys. 103, 10387 (1995).

[9] T. Antal, M. Droz, J. Magnin, Z. Rácz, and M. Zrínyi, J. Chem. Phys. 109, 9479 (1998).

[10] F. Izsak and I. Lagzi, Models of Liesegang Pattern Formation, Department of Applied Mathematics (University of Twente, Department of Applied Mathematics, 2010).

[11] M. Droz, J. Stat. Phys. 101, 509 (2000).

[12] M. R. Tinsley, D. Collison, and K. Showalter, J. Phys. Chem. A 117, 12719 (2013).

[13] M. Tinsley, D. Collison, and K. Showalter, Chaos 25, 064306 (2015).

[14] M. M. Ayass, I. Lagzi, and M. Al-Ghoul, Phys. Chem. Chem. Phys. 16, 24656 (2014).

[15] A. Volford, F. Izsák, M. Ripszám, and I. Lagzi, Langmuir 23, 961 (2007). 
[16] S.Thutupalli, Towards Autonomous Soft Matter Systems, Ph.D. thesis, University of Göttingen (2014).

[17] S. Herminghaus, C. C. Maass, C. Krüger, S. Thutupalli, L. Goehring, and C. Bahr, Soft Matter 10, 7008 (2014).

[18] Y. Matsuda, N. J. Suematsu, H. Kitahata, Y. S. Ikuro, and S. Nakata, Chem. Phys. Lett. 654, 92 (2016).

[19] A. Diguet, R.-M. Guillermic, N. Magome, A. Saint-Jalmes, Y. Chen, K. Yoshikawa, and D. Baigl, Angew. Chem., Int. Ed. 48, 9281 (2009).

[20] Z. Izri, M. N. van der Linden, S. Michelin, and O. Dauchot, Phys. Rev. Lett 113, $248302(2014)$.

[21] S. J. S. Jamaluddin, K. Khaothong, M. R. Tinsley, and K. Showalter, Chaos 30, 083143 (2020).

[22] S. Kitawaki, K. Shioiri, T. Sakurai, and H. Kitahata, J. Phys. Chem. C 116, 26805 (2012). 


\section{Chapter 2}

\section{The Belousov-Zhabotinsky Reaction}

\section{$2.1 \quad$ Introduction}

In 1950, a Russian scientist named Boris Belousov was working on the Krebs cycle. He was trying to model the catalysis in the Krebs cycle. He swapped the protein-bound metal ion that is usually found in the enzymes with cerium ion. Surprisingly, the color of his solution oscillated between colorless and yellow while the solution was stirred continuously. When the solution was left unstirred, he observed yellow traveling waves in the reaction medium. ${ }^{1]}$ He documented this observation, and he systematically studied this system. He was hoping that he had discovered something novel, and he could publish his findings in a respectable journal. However, to his dismay he only managed to publish his finding in an obscure medical journal.

Several years later, Belousov's chemical mixture recipe was discovered by Anatol

Zhabotinsky, who was then a graduate student at Moscow State University. ${ }^{[2]}$ He improved Belousov's chemical mixture recipe by substituting citric acid with malonic acid. He also studied the system under unstirred condition. ${ }^{[3}$ Since then this reaction has been dubbed the Belousov-Zhabotinsky (BZ) reaction in honor of these two scientists, and the reaction has 
been studied extensively by scientists around the world.

In a continuously stirred BZ solution, the color of the solution oscillates between two different colors depending on the catalyst used in the solution. With cerium ion as the catalyst, the color of the solution oscillates between colorless $\left(\mathrm{Ce}^{3+}\right)$ and yellow $\left(\mathrm{Ce}^{4+}\right)$. If ferroin is used to catalyze the reaction, the color of the solution oscillates between red $\left(\mathrm{Fe}^{2+}\right)$ and blue $\left(\mathrm{Fe}^{3+}\right)$. On the other hand, the solution color will oscillate between orange $\left(R u^{2+}\right)$ and green $\left(R u^{3+}\right)$ when tris(bipyridine)ruthenium(II) chloride is used to catalyze the reaction.

In order to develop a deeper understanding of the BZ reaction, several mechanisms have been proposed to explain the temporal oscillations exhibited by the stirred BZ solution. Belousov tried to provide some mechanistic explanations on the origin of the temporal oscillations in his unpublished paper. His work was continued by Zhabotinsky. ${ }^{[2]}$ However, the more prevalent mechanistic explanation of the BZ solution temporal oscillations was provided by Field, Körös, and Noyes in 1971.415

\subsection{The Field-Körös-Noyes (FKN) Mechanism}

The FKN mechanism was developed to provide a mechanistic understanding of the temporal oscillations of the BZ reaction. $\frac{45}{45}$ The $\mathrm{BZ}$ reaction mechanism consists of a collection of 10 reactions, which can be grouped into three processes: process $\mathrm{A}$, process $\mathrm{B}$, and

process $C .{ }^{6}$ Process A is essentially the reduction of bromate ion by bromide ion through a 


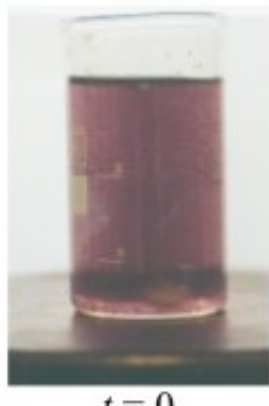

$t=0$

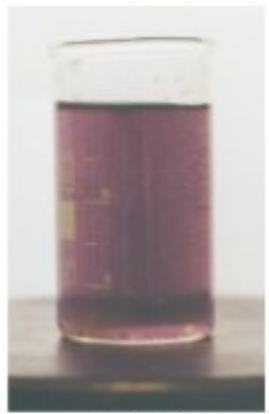

$t=25 \mathrm{~s}$

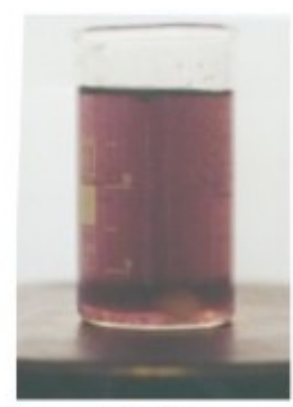

$t=5 \mathrm{~s}$

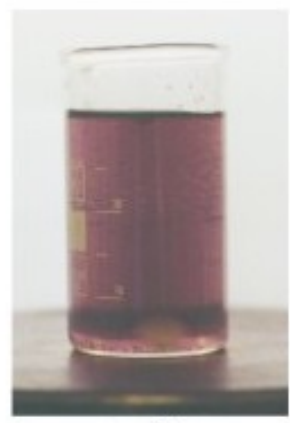

$t=30 \mathrm{~s}$

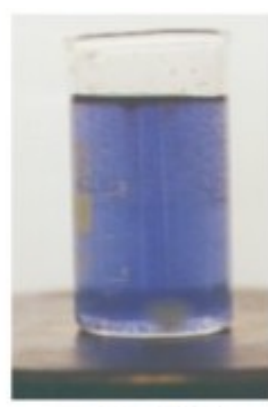

$t=10 \mathrm{~s}$

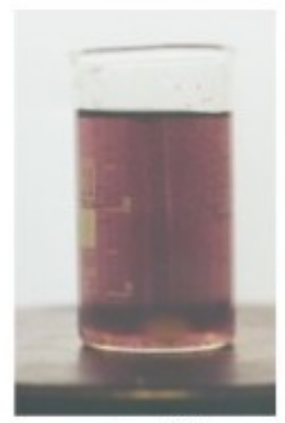

$t=35 \mathrm{~s}$

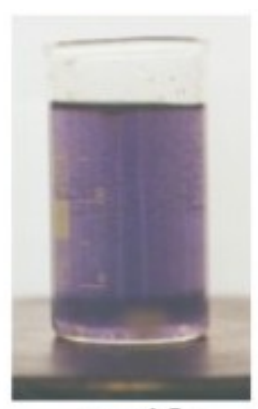

$t=15 \mathrm{~s}$

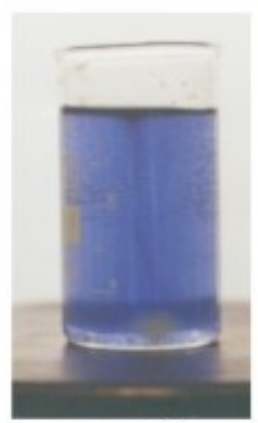

$t=40 \mathrm{~s}$

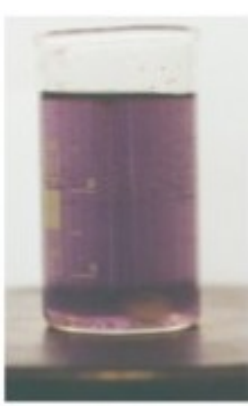

$t=20 \mathrm{~s}$

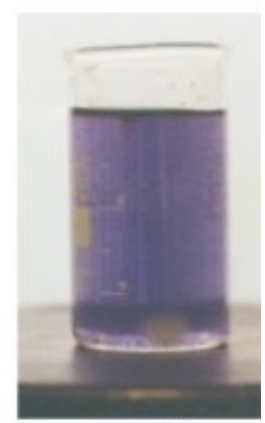

$t=45 \mathrm{~s}$

Figure 2.1: A series of images of a stirred BZ solution. Images from Wikipedia.

series of oxygen transfers:

$$
\begin{aligned}
& \mathrm{Br}^{-}+\mathrm{BrO}_{3}^{-}+2 \mathrm{H}^{+} \longrightarrow \mathrm{HBrO}_{2}+\mathrm{HOBr} \\
& \mathrm{Br}^{-}+\mathrm{HBrO}_{2}+\mathrm{H}^{+} \longrightarrow 2 \mathrm{HOBr} \\
& \mathrm{Br}^{-}+\mathrm{HOBr}+\mathrm{H}^{+} \longrightarrow \mathrm{Br}_{2}+\mathrm{H}_{2} \mathrm{O} \\
& \mathrm{Br}_{2}+\mathrm{CH}_{2}(\mathrm{COOH})_{2} \longrightarrow \mathrm{BrCH}(\mathrm{COOH})_{2}+\mathrm{Br}^{-}+\mathrm{H}^{+}
\end{aligned}
$$

By combining $R 1+R 2+3 R 3+3 R 4$, process A can be summarized as follows:

$$
\mathrm{BrO}_{3}^{-}+2 \mathrm{Br}^{-}+3 \mathrm{CH}_{2}(\mathrm{COOH})_{2}+3 \mathrm{H}^{+} \longrightarrow 3 \mathrm{BrCH}(\mathrm{COOH})_{2}+3 \mathrm{H}_{2} \mathrm{O} \text {. }
$$

Process $\mathrm{A}$ is responsible for consuming the bromide ions in the solution. Process A will dominate the reaction dynamics as long as the concentration of bromide remains above a critical concentration. If the concentration of bromide ions drops below its critical concentration, process $\mathrm{B}$ will start to dominate the overall chemical dynamics of the BZ reaction. 
Process B is characterized by the change in the color of the BZ solution. The color change is caused by the oxidation of the metal catalyst in the BZ reaction. The following reactions make up process B:

$$
\begin{aligned}
\mathrm{BrO}_{3}^{-}+\mathrm{HBrO}_{2}+\mathrm{H}^{+} & \longrightarrow 2 \mathrm{BrO}_{2}^{\bullet}+\mathrm{H}_{2} \mathrm{O}, \\
\mathrm{M}_{\text {red }}+\mathrm{BrO}_{2}^{\bullet}+\mathrm{H}^{+} & \longrightarrow \mathrm{M}_{\text {ox }}+\mathrm{HBrO}_{2}, \\
2 \mathrm{HBrO}_{2} & \longrightarrow \mathrm{HOBr}+\mathrm{BrO}_{3}^{-}+\mathrm{H}^{+}, \\
\mathrm{HOBr}+\mathrm{CH}_{2}(\mathrm{COOH})_{2} & \longrightarrow \mathrm{BrCH}(\mathrm{COOH})_{2}+\mathrm{H}_{2} \mathrm{O} .
\end{aligned}
$$

Another hallmark of process B is the autocatalytic reaction that occurs in process B. The autocatalytic reaction is the result of $(\mathrm{R} 5)+2(\mathrm{R} 6)$. With these reactions one can see that for every $\mathrm{HBrO}_{2}$ consumed, $2 \mathrm{HBrO}_{2}$ are produced. The autocatalytic reaction results in the rapid production of $\mathrm{HBrO}_{2}$.

$$
\begin{gathered}
\mathrm{BrO}_{3}^{-}+\mathrm{HBrO}_{2}+\mathrm{H}^{+} \longrightarrow 2 \mathrm{BrO}_{2}^{\bullet}+\mathrm{H}_{2} \mathrm{O}, \\
2 \mathrm{M}_{r e d}+2 \mathrm{BrO}_{2}^{\bullet}+2 \mathrm{H}^{+} \longrightarrow 2 \mathrm{M}_{o x}+2 \mathrm{HBrO}_{2}, \\
\mathrm{BrO}_{3}^{-}+\mathrm{HBrO}_{2}+3 \mathrm{H}^{+}+2 \mathrm{M}_{\text {red }} \longrightarrow 2 \mathrm{M}_{o x}+\mathrm{H}_{2} \mathrm{O}+2 \mathrm{HBrO}_{2} .
\end{gathered}
$$

Process B can be summarized by combining $2(R 5)+4(R 6)+(R 4)+(R 8 a)$ as follows:

$$
\mathrm{BrO}_{3}^{-}+4 \mathrm{M}_{\text {red }}+\mathrm{CH}_{2}(\mathrm{COOH})_{2}+5 \mathrm{H}^{+} \longrightarrow \mathrm{BrCH}(\mathrm{COOH})_{2}+4 \mathrm{M}_{\text {ox }}+3 \mathrm{H}_{2} \mathrm{O} \text {. }
$$

Process $\mathrm{C}$ can be thought of as a collection of reactions that act as a resetting process, since the reduced form of the metal catalyst is regenerated:

$$
\begin{gathered}
6 \mathrm{M}_{o x}+\mathrm{CH}_{2}(\mathrm{COOH})_{2}+2 \mathrm{H}_{2} \mathrm{O} \longrightarrow 6 \mathrm{M}_{r e d}+\mathrm{HCOOH}+2 \mathrm{CO}_{2}+6 \mathrm{H}^{+}, \\
4 \mathrm{M}_{\text {ox }}+\mathrm{BrCH}(\mathrm{COOH})_{2}+2 \mathrm{H}_{2} \mathrm{O} \longrightarrow \mathrm{Br}^{-}+4 \mathrm{M}_{\text {red }}+\mathrm{HCOOH}+2 \mathrm{CO}_{2}+5 \mathrm{H}^{+} .
\end{gathered}
$$


The overall BZ reaction can be written as

$$
3 \mathrm{BrO}_{3}^{-}+5 \mathrm{CH}_{2}(\mathrm{COOH})_{2}+3 \mathrm{H}^{+} \longrightarrow 3 \mathrm{BrCH}(\mathrm{COOH})_{2}+2 \mathrm{HCOOH}+4 \mathrm{CO}_{2}+5 \mathrm{H}_{2} \mathrm{O} \text {. }
$$

\subsection{Self-organized Patterns in the Unstirred BZ Reaction}

One dimensional unstirred BZ solutions can be created by having a thin tube filled with BZ solution. ${ }^{3}$ In this system, traveling wave fronts will propagate from the initiation point toward the end of the tube. However, if there are two initiation points in the tube, traveling wave fronts from the initiation points will collide and mutually annihilate. If one of the initiation points generates traveling waves faster than the other, the dynamic of the initiation point with higher frequency will dominate the reaction medium. This causes the entire tube to be filled with traveling waves from the initiation points with higher frequency. ${ }^{3}$

The simplest setup for a two dimensional unstirred BZ solution involves a thin layer of BZ solution, approximately $1-2 \mathrm{~mm}$ in depth, in a petri dish.7 ${ }^{7}$ This is a two-dimensional closed system of the BZ reaction. This setup allows the formation of patterns such as target patterns and spiral waves patterns. However, since this is a closed system, the reaction can only be sustained for a short amount of time.

A two-dimensional open system of the BZ reaction can be created by using a reactor

with two reservoirs separated by a thin porous glass disk. ${ }^{810}$ Each reservoir typically contains an incomplete chemical mixture of the BZ reaction. However, the chemical mixture from each reservoir can diffuse into the porous glass and react to produce patterns. Fresh solution is continuously pumped into each reservoir and the reaction by-products are pumped out of the reservoir to sustain the chemical reaction that is occurring in the porous glass. The 
porous glass used is usually very thin in order to prevent three-dimensional effects from interfering with the experiment. Since mixing is prevented by the porous glass, the reaction within the porous glass relies on diffusion of the chemical species for transport.

Similar to the one-dimensional system, a two-dimensional system can have waves propagating from a single initiation center. However, rather than having multiple bands propagating from the initiation points, the waves take a form of concentric rings expanding from the initiation point. This multiple rings pattern is usually referred to as a target pattern.

Typical one-dimensional wave behaviors had been observed in the two-dimensional system. Mutual wave annihilation also occurs in the two-dimensional system when waves from different initiation centers meet. If one of the initiation centers generates waves at a higher rate than the others, the waves from this initiation center will dominate the entire reaction medium. If any part of the target patterns is broken, the broken parts can develop into spiral waves. ${ }^{7}$

Spiral wave originates from a spiral wave tip. The spiral wave tip typically revolves around a core, which remains unexcited.11 As the spiral wave tip revolves around its core, the tip sends out waves, which travel outward. If the spiral wave tip rigidly revolves around the core, simple spiral waves are formed. However, the tip of the spiral wave can start to meander in a flower-like trajectory when the initial concentration of malonic acid in the solution is lowered. $10[12$

As the spiral wave tip meanders, the spiral waves in front of the tip are compressed while spiral waves behind the tips are dilated. The compression and the dilation of the spiral waves is called the Doppler effect. If the concentration of the malonic acid is lowered beyond the second critical concentration, the system transitions to the turbulence state. The turbulence state is characterized by the spiral instability. Under this condition, spiral waves 
near the center break and spontaneously generates new spiral wave tips (defects). The newly generated spiral wave tips create new sets of spiral waves. The entire process of spiral wave breakage, formation of new spiral wave tips, and creation of new spiral waves is repeated over and over again until the entire reaction medium is filled with defects. 1012

The next level of complexity is a three-dimensional system. Unlike a two-dimensional system, it is hard to generate a controlled vortex initiation in a three-dimensional system. One way of generating a controlled vortex is using a double layer gel containing the BZ mixture. 13 The first layer of the liquid gel is typically poured into a container followed by the solidification of the liquid gel. A circular wave is then initiated, and after the circular wave has travelled for a certain distance, a second layer of liquid gel is poured on top of the first layer gel. The addition of the second layer gel provides the system with fresh reagents and reaction medium. This allows the initial wave front to curl up and develop into a scroll wave.

\subsection{Mathematical Modeling of the $\mathrm{BZ}$ reaction}

\subsubsection{The Oregonator Model}

The 10 elementary reactions of the FKN model can be hard to model. Therefore, there is a need to create a simpler model while simultaneously preserving the critical features of the BZ reaction. The FKN mechanism showed that there is a competition between the bromide and the bromate ions for bromous acid. This competition dictates which process will dominate the reaction dynamics of the BZ system. If the concentration of bromide ions is above its critical value, bromide ions will outcompete bromate ions to react with the bromous acid. In this case, process A dominates the reaction dynamics. If the concentration of bromide ions is below its critical value, bromate ions will outcompete bromide ions for 
bromous acid resulting in process $\mathrm{B}$ dominating the reaction dynamics.

A simpler model was developed in 1974 that consists of five reactions while preserving the critical features of the BZ reaction. This model was called the Oregonator, and it was proposed by Field and Noyes. $\stackrel{14}{14}$ The model is as follows:

$$
\begin{aligned}
A+Y & \longrightarrow X, \\
X+Y & \longrightarrow P, \\
A+X & \longrightarrow 2 X+Z, \\
2 X & \longrightarrow Q, \\
Z & \longrightarrow f Y,
\end{aligned}
$$

where $\mathrm{X} \equiv \mathrm{HBrO}_{2}, Y \equiv \mathrm{Br}^{-}, Z \equiv M_{o x}, A \equiv \mathrm{BrO}_{3}^{-}$, and $f$ is a stoichiometric factor. The kinetics of the Oregonator model can be described by the following set of differential equations:

$$
\begin{aligned}
\frac{d X}{d t} & =k_{O 1} A Y-k_{O 2} X Y+k_{O 3} A X-2 k_{O 4} X^{2} \\
\frac{d Y}{d t} & =-k_{O 1} A Y-k_{O 2} X Y+f k_{O 5} Z \\
\frac{d Z}{d t} & =k_{O 3} B X-k_{O 5} Z
\end{aligned}
$$

Equations 2.3 to 2.5 can be integrated to find the solutions to the Oregonator model. Figure 2.2 shows the solution from integrating equations 2.3 to 2.5 using ode15s solver in MATLAB. The sudden drops in bromide concentration in Figure 2.2(b) represent the time when process $\mathrm{B}$ of the $\mathrm{BZ}$ reaction starts to dominate the kinetics.

\subsubsection{The Reaction-Diffusion Model}

A general differential equation describing the reaction-diffusion phenomena for an unstirred BZ solution can be constructed by including the diffusion term into the existing 

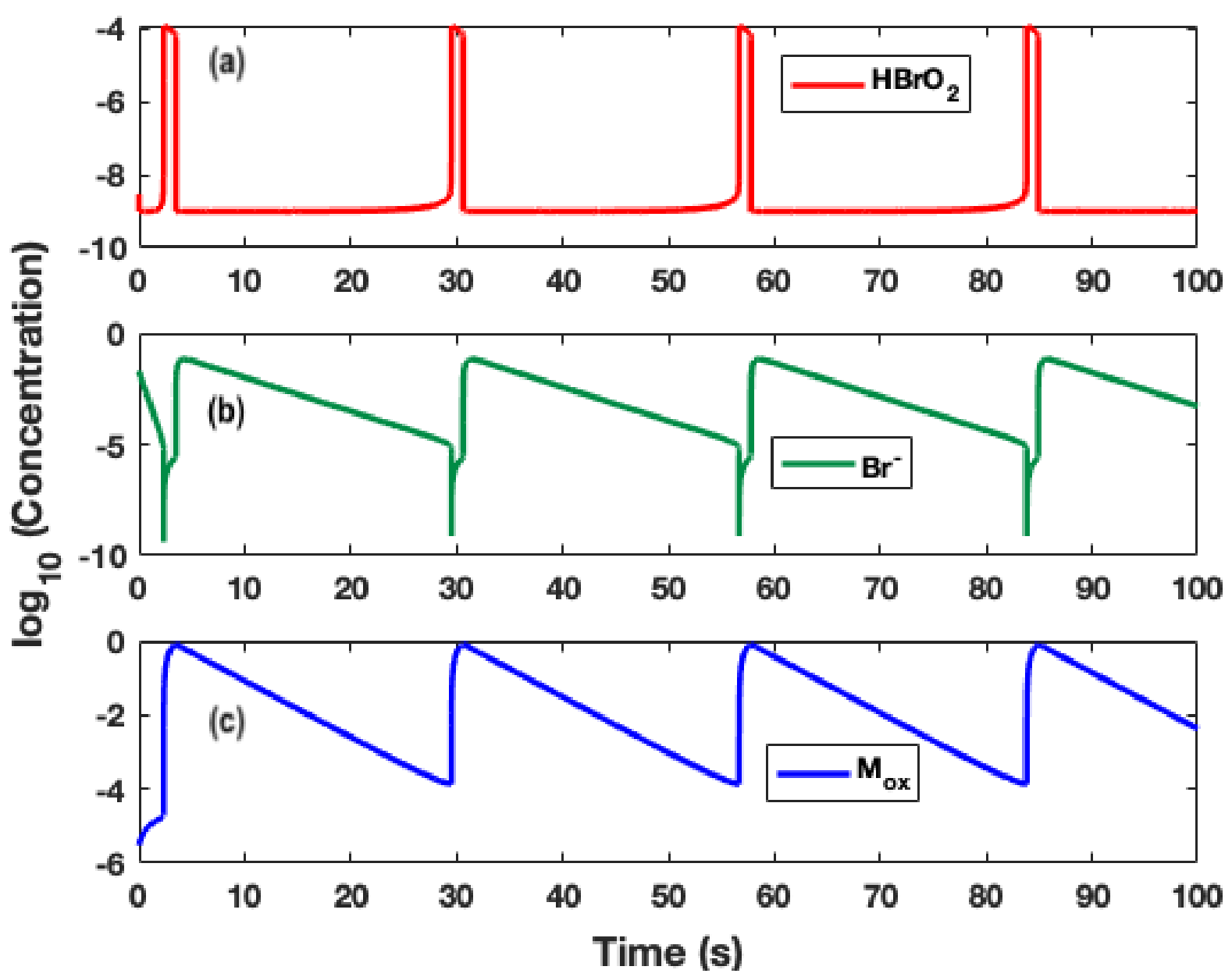

Figure 2.2: Plots of $\log _{10}$ (Concentration) as a function of time based on the Oregonator model for (a) bromous acid, (b) bromide ions, and (c) oxidized metal catalyst. The periodic sudden drop in bromide concentration represents the start of process B dominating the BZ reaction.

reaction differential equations

$$
\frac{\partial u_{n}}{\partial t}=D_{n} \nabla^{2} u_{n}+f\left(u_{n}\right)
$$

where $u_{n}$ is the concentration of $n^{\text {th }}$ species, $D_{n}$ is the diffusion coefficient of $n^{\text {th }}$ species, and $f\left(u_{n}\right)$ is the function that describes the reaction kinetics involving $n^{\text {th }}$ species. The number of $n$ species and $f\left(u_{n}\right)$ depends on the models used.

Keener and Tyson had modeled the formation of spiral waves based on Oregonator model. By assuming that the concentration of bromate and the concentration of bromoma- 
lonic acid plus malonic acid to be constant, the resulting mass action kinetics consists of three chemical species, bromous acid, metal catalyst, and bromide ion, and their associated diffusion coefficients. ${ }^{15}$ By solving the resulting differential equations, they showed that the curvature of spiral wave fronts affects its normal velocity. They also showed that their theory matched the experimental observation of that time. $\frac{15}{.5}$

\subsection{The Photosensitive Catalyst}

Throughout the years several different catalysts have been used to catalyze the BZ reaction. The catalysts used include $C e^{3+}, \mathrm{Mn}^{2+}$, ferroin, and $R u(b p y)_{3}^{2+}$. Unlike most of the other catalysts, $R u(b p y)_{3}^{2+}$ is photosensitive. $R u(b p y)_{3}^{2+}$ has been shown to increase the $\mathrm{BZ}$ reaction oscillation period. If the light intensity is increased beyond the critical value, the chemical oscillations can be suppressed.16117

Studies showed that photosensitivity of $R u(b p y)_{3}^{2+}$ comes in two forms: photoinhibitory and photoexcitatory. In both cases, $R u(b p y)_{3}^{2+}$ is first promoted to its excited state, $R u(b p y)_{3}^{2+*}$, by irradiation of $452 \mathrm{~nm}$ light. The $R u(b p y)_{3}^{2+*}$ is a strong reducing agent, which can reduce other chemical species present in the BZ solution.

In the case of photoinhibitory, the following reactions take place

$$
\begin{aligned}
R u(b p y)_{3}^{2+}+h \nu & \rightleftharpoons R u(b p y)_{3}^{2+*}, \\
R u(b p y)_{3}^{2+*}+B r M A & \rightarrow R u(b p y)_{3}^{3+}+B r^{-}+\text {org. prod }, \\
R u(b p y)_{3}^{3+}+B r M A & \rightarrow R u(b p y)_{3}^{2+}+B r^{-}+\text {org. prod },
\end{aligned}
$$

where $h \nu$ and BrMA are photons at $452 \mathrm{~nm}$ and bromomalonic acid, respectively. Equation 2.7 is the excitation reaction of the ground state catalyst, $R u(b p y)_{3}^{2+}$, to its excited state, $R u(b p y)_{3}^{2+*}$. Equations 2.8 and 2.9 show the production of bromide ion from bromomalonic 
acid by the excited catalyst. The production of bromide ion from these two reactions increases the concentration of bromide ions in the solution, thus allowing process $\mathrm{A}$ of the BZ reaction to dominate the reaction dynamics again. 16

In the case of photoexcitatory, the first step involves the promotion of $R u(b p y)_{3}^{2+}$ to $R u(b p y)_{3}^{2+*}$ similar to reaction 2.7. This is followed by the reduction of bromate to $\mathrm{BrO}_{2}^{\bullet}$ as follows

$$
\mathrm{Ru}(b p y)_{3}^{2+*}+\mathrm{BrO}_{3}^{-}+2 \mathrm{H}^{+} \rightarrow \mathrm{BrO}_{2}^{\bullet}+\mathrm{H}_{2} \mathrm{O}+\mathrm{Ru}(b p y)_{3}^{3+}
$$

The radical $\mathrm{BrO}_{2}^{\bullet}$ then can be part of the autocatalytic reactions $\mathrm{R} 5$ and $\mathrm{R} 6$ to generate $\mathrm{HBrO}_{2}$. The photoinhibitory process can be achieved using high illumination intensity while the photoexcitatory process can be achieved using low illumination intensity of $452 \mathrm{~nm}$ light.

In order to take into account the effect of light on the catalyst, two additional reactions are added to the Oregonator model as follows:

$$
\begin{array}{r}
E+V \longrightarrow Y+Z, \\
E+A \longrightarrow X+2 Z,
\end{array}
$$

where $\mathrm{E}$ is the excited metal catalyst concentration and $\mathrm{V}$ is the bromomalonic acid concentration. Equation $\mathrm{P} 1$ and equation $\mathrm{P} 2$ represent $\mathrm{Br}^{-}$production and $\mathrm{HBrO}_{2}$ production, respectively. The resulting differential equations that include these additional reactions are 
as follow:

$$
\begin{aligned}
& \frac{[A]}{d t}=-k_{O 1}[A][Y]-k_{O 3}[A][X]+k_{O 4}[X]^{2}-k_{P 2}[E][A], \\
& \frac{[X]}{d t}=k_{O 1}[A][Y]-k_{O 2}[X][Y]+k_{O 3}[A][X]-2 k_{O 4}[X]^{2}+k_{P 2}[E][A], \\
& \frac{[Y]}{d t}=-k_{O 1}[A][Y]-k_{O 2}[X][Y]+h k_{O 5}[M][Z]+k_{P 1}[E][V], \\
& \frac{[Z]}{d t}=2 k_{O 3}[A][X]-k_{O 5}[M][Z]+k_{P 1}[E][V]+2 k_{P 2}[E][A], \\
& \frac{[M]}{d t}=-k_{O 5}[M][Z], \\
& \frac{[V]}{d t}=-k_{P 1}[E][V],
\end{aligned}
$$

where equations 2.11 through 2.16 can be integrated to simulate the effect of photosensitive catalyst on the BZ reaction. 


\subsection{References}

[1] J. Miyazaki, in Pattern Formations and Oscillatory Phenomena, edited by S. Kinoshita (Elsevier, 2013) pp. 61-77.

[2] A. T. Winfree, J. Chem. Educ. 61, 661 (1984).

[3] A. N. Zaikin and A. M. Zhabotinsky, Nature 225, 535 (1970).

[4] R. M. Noyes, R. J. Field, and E. Körös, J. Am. Chem. Soc. 94, 1394 (1971).

[5] R. Field, E. Körös, and R. Noyes, J. Am. Chem. Soc. 94, 8649 (1972).

[6] V. Voorsluijs, I. G. Kevrekidis, and Y. D. Decker, Phys. Chem. Chem. Phys. 19, 22528 (2017).

[7] A. F. Taylor, Prog. React. Kinet. Mech. 27, 247 (2002).

[8] A. Belmonte, J. M. Flesselles, and Q. Ouyang, Europhys. Lett. 35, 665 (1996).

[9] Q. Ouyang and J. M. Flesselles, Nature 379, 143 (1996).

[10] Q. Ouyang, H. L. Swinney, and G. Li, Phys. Rev. Lett. 84, 1047 (2000).

[11] G. Biosa, S. Bastianoni, and M. Rustici, Chem. - Eur. J. 12, 3430 (2006).

[12] H. Guo, L. Li, Q. Ouyang, J. Liu, and Z. She, J. Chem. Phys. 118, 5038 (2003).

[13] A. Winfree and W. Jahnke, J. Phys. Chem. 93, 2823 (1989).

[14] R. J. Field and R. M. Noyes, J. Chem. Phys. 60, 1877 (1974).

[15] J. P. Keener and J. J. Tyson, Phys. D 21, 307 (1986).

[16] S. Kádár, T. Amemiya, and K. Showalter, J. Phys. Chem. A 101, 8200 (1997). 
[17] S. Kitawaki, K. Shioiri, T. Sakurai, and H. Kitahata, J. Phys. Chem. C 116, 26805 (2012).

[18] I. Hanazaki and Y. Mori and T. Sekiguchi and G. Rábai, Phys. D 84, 228 (1995). 


\section{Chapter 3}

\section{Belousov-Zhabotinsky Droplet}

\section{Motion Control Using a Light Intensity Gradient}

\subsection{Introduction}

The Belousov-Zhabotinsky (BZ) reaction is one of the most studied chemical reactions. The color of the solution is known to oscillate temporally when the solution is stirred continuously. In an unstirred reaction vessel, the BZ solution is known to produce spatial patterns such as target, spiral, and scroll wave patterns. ${ }^{112}$ A working mechanism for temporal oscillation of the BZ reaction was proposed by Field, Körös, and Noyes in 1972. The

mechanism consists of three main processes: process A, process B, and process C. Process A involves the consumption of bromide ions in acidic bromate solution to produce bromine molecules. The bromine molecules then react with malonic acid to produce bromomalonic acid, which is used in process C. Process B involves the oxidation of the metal catalyst in the 
solution. This process is accompanied by the solution color change due to the catalyst oxidation. Process $\mathrm{C}$ involves resetting the reaction clock by the reduction of the oxidized metal catalyst. Process $\mathrm{C}$ is accompanied by the color change back to its reduced state color. $\frac{3,4}{3}$ The change in the catalyst oxidation state can affect its reaction medium properties such as its color, surface tension, and hydrophobicity. ${ }^{[57}$ The change in the medium properties has been exploited by researchers to create self-propelled particles such as aqueous BZ droplets in an oil-phase. $\frac{899}{8}$

The BZ droplets are produced by introducing a small amount of the BZ solution into an oil-phase. The oil-phase can be made up of only oil such as oleic acid, or it can be

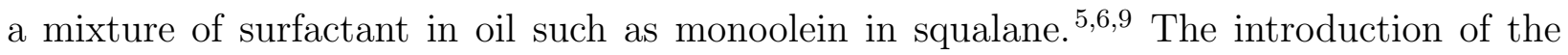
BZ solution into the oil-phase can be achieved in several ways. One way is to pipet the BZ solution directly into the oil-phase using a micropipet. $\stackrel{5[6] 10}{ }$ This method is typically used to manufacture BZ droplets with a diameter of greater than $1000 \mu \mathrm{m}$. This method is preferred for its simplicity in producing BZ droplets without a complicated setup. For a smaller size BZ droplet production, microfluidic chips are often utilized. This method involves pumping the BZ solution via a microfluidic chip as the discrete phase into the oil-phase, which acts as the continuous phase. This method allows for the production of BZ droplets as small as a few tens of microns. The size of the BZ droplets can be adjusted by varying the flow rate of the continuous and the discrete phase. .911

In the absence of the surfactant, the propulsion of the BZ droplets relies on the surface tension gradient created by the difference in the catalyst oxidation state. Regions on the BZ droplet surface with the oxidized catalyst will have a higher surface tension relative to regions with the reduced catalyst. ${ }^{6}$ In the presence of the surfactant, the surface tension gradient is created by modifying the chemical structure of the surfactant. 11 13 In the case where monoolein is utilized as the surfactant, the double bond of the monoolein can be 
brominated. The bromination of the monoolein double bond causes its surface tension to increase from $1.3 \mathrm{mN} \mathrm{m}^{-1}$ to $2.7 \mathrm{mN} \mathrm{m}^{-1}$.1112 Surface tension manipulation via a surfactant property is not limited to the BZ droplet system since other systems such as oleic acid on aqueous azobenzene trimethylammonium bromide system have been shown to behave similar to the BZ droplet system. $\frac{13}{13}$

Several studies have been conducted to elucidate the propulsion mechanism of the BZ droplet. In the case of the BZ droplet in a monoolein/squalane mixture, the propulsion mechanism assumes that the BZ droplet surface is initially covered with the monoolein. Within the BZ droplet, bromine molecules are produced by the process A of the BZ reaction. ${ }^{3}$ The bromine molecules then react with the double bond of the monoolein. The bromination of the double bond changes the surface activity of the surfactant. Regions with predominantly brominated monoolein will have a higher surface tension relative to the regions with predominantly fresh monoolein. $\frac{9|11| 12}{10}$ The creation of the surface tension gradient causes fluid to flow from low surface tension regions to high surface tension regions. The fluid flow on the surface of the BZ droplet creates convective flow within and surrounding the BZ droplet. Due to the momentum transfer from the BZ droplet to the surrounding fluid, the BZ droplet is propelled in the opposite direction of the fluid flow.

By manipulating the monoolein bromination rate, it is possible to control the BZ droplet motion. In the BZ droplet system, the bromination rate of the monoolein depends on the production of bromine by the BZ reaction. Therefore, if the BZ reaction kinectics can be manipulated, it is possible to control the BZ droplet motion. One way of achieving this is by using a photosensitive catalyst, tris(2,2'-bipyridyl)ruthenium(II)-catalyst, $R u(b p y)_{3}^{2+}$, which responds to $452 \mathrm{~nm}$ light. $.1415 \mathrm{In}$ In this chapter, the motion control of the BZ droplet immersed in a monoolein/squalane mixture is demonstrated by utilizing a light intensity gradient. The accuracy of the BZ droplet motion control is discussed in terms of the BZ droplet trajectory 
and the angle $\theta$ distribution. A simulation on the effect of light intensity on the reduced and the oxidized steady-state BZ reaction is included to explain the difference in behavior between the reduced and the oxidized steady-state BZ droplet.

\subsection{Experimental Method}

The BZ solution is prepared by mixing malonic acid (MA), sodium bromate $\left(\mathrm{NaBrO}_{3}\right)$, sulfuric acid $\left(\mathrm{H}_{2} \mathrm{SO}_{4}\right)$, sodium bromide $(\mathrm{NaBr})$, and tris $(2,2$ '-bipyridyl)ruthenium(II) catalyst to the desired concentrations. The oil-phase is prepared by dissolving the surfactant, monoolein, in water saturated squalane to achieve a $10 \mathrm{mM}$ monoolein/squalane solution. The reaction vessel is made hydrophobic by applying a commercially available water repellent

product, RainX, on the inner surface of the reactor. Figure 3.1(a) shows the experimental setup used in this study. The BZ droplet is produced by pipetting $\sim 0.6 \mu \mathrm{L}(\sim 0.6 \mathrm{~mm}$ radius) of the $\mathrm{BZ}$ solution mixture into the oil-phase, which is $4 \mathrm{~mm}$ deep. A camera is used to record the movement of the BZ droplet in the reaction vessel. The illumination on the particle is produced using a projector. The camera and the projector are connected to a computer. The computer is loaded with MATLAB, which allows for real-time droplet detection and light intensity gradient placement.

\subsection{Results}

\subsubsection{Homogeneous Light Intensity}

The behavior of the BZ droplet is initially studied using a homogeneous light intensity. In order to characterize the behavior of the BZ droplet, the distribution of the angle $\theta$ is used. The angle $\theta$ can be interpreted as the instantaneous velocity direction of the BZ 


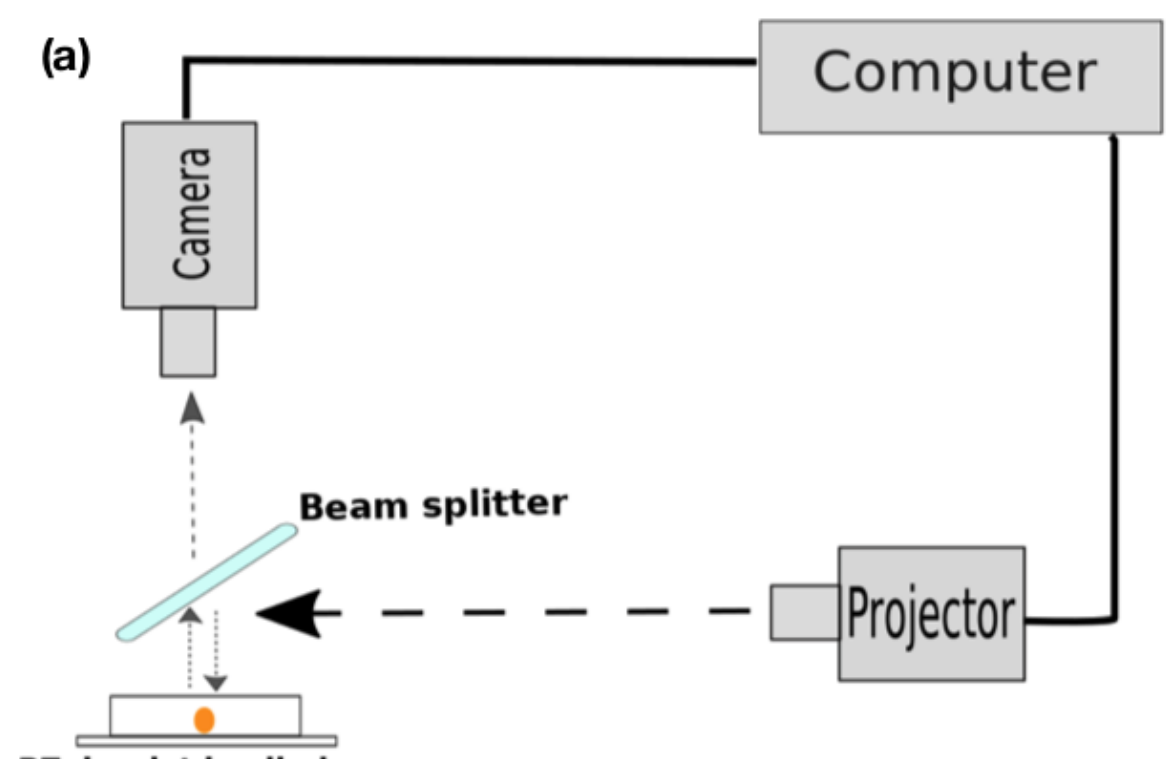

BZ droplet in oil-phase

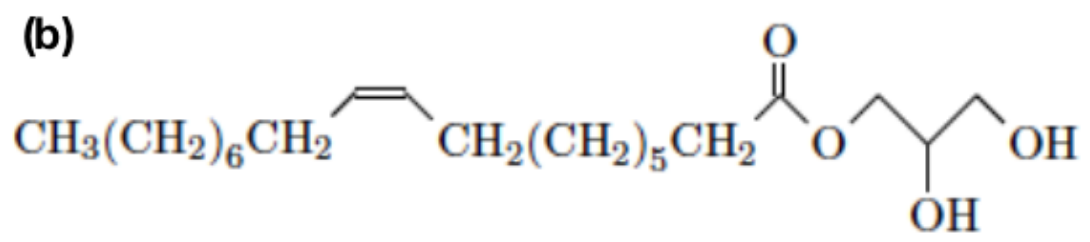

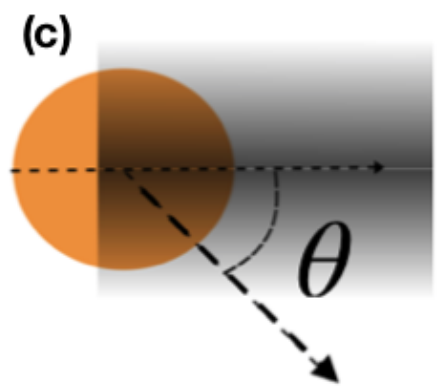

(d)

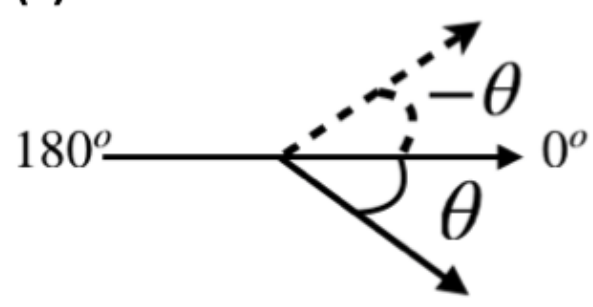

Figure 3.1: (a) The experimental setup used in controlling the BZ droplet motion in a monoolein/squalane mixture. (b) Chemical structure of the surfactant, monoolein. (c) The trajectory angle $\theta$ definition. The angle $\theta$ range is $\left(-180^{\circ} \cup 180^{\circ}\right]$. Images taken from reference [10].

droplet with respect to the positive direction of the x-axis. Figure 3.1(c) and (d) show the definition and the range of the angle $\theta$, respectively. The value of the angle $\theta$ is restricted to $\left(-180^{\circ} \cup 180^{\circ}\right]$ to avoid double counting the backward movement of the BZ droplet.

The results from homogeneous light intensity experiments for the reduced and the 
oxidized steady-state of the BZ droplet are shown in column (a) and column (b) of Figure 3.2. respectively. The trajectory for the reduced and the oxidized steady-state of the BZ droplet shows that the BZ droplet does not have a preferential direction when it is exposed to the homogeneous light intensity. This behavior is further confirmed by the angle $\theta$ distribution of the BZ droplet for both cases, which shows that the distribution is relatively flat. This signifies that there is no preferential direction when the BZ droplet is exposed to the homogeneous light intensity. However, the average speed of the BZ droplet for the reduced and the oxidized steady-state are different. The average BZ droplet speed for the reduced steady-state is $79 \mu \mathrm{m} \mathrm{s}^{-1}$ while the average speed for the oxidized steady-state BZ droplet is $47 \mu \mathrm{m} \mathrm{s} \mathrm{s}^{-1}$.

\subsubsection{Constant Light Intensity Gradient}

The next set of experiments involves applying a constant light intensity gradient on the BZ droplet to demonstrate the ability to control the BZ droplet movement using a light intensity gradient. In this set of experiments, a constant light intensity gradient is applied on the BZ droplet. The light intensity gradient is applied using a control box similar to Figure 3.3(a). The control box is constructed based on the following equation: $\phi(x, y)=$ $c_{1}\left(x-x_{0}\right)+c_{0}, \forall y$ and $-W / 2 \leqslant\left(x-x_{0}\right) \leqslant W / 2$, where $\phi(x, y)$ is the local illumination intensity within the control box, $c_{0}=4.81 \times 10^{-4} \mathrm{~W} \mathrm{~cm}^{-2}, c_{1}=-1.28 \times 10^{-3} \mathrm{~W} \mathrm{~cm}^{-3}, x_{0}$ is the current $\mathrm{x}$-coordinate of the center of the $\mathrm{BZ}$ droplet, and $W=0.34 \mathrm{~cm}$ is the width of the applied light intensity gradient. The length of the box spanned the entire experimental area. Outside of the control box, the light intensity is maintained at $9.09 \times 10^{-4} \mathrm{~W} \mathrm{~cm}^{-2}$. The dashed line in Figure 3.3(a) indicates the center of the control box for this set of experiments. The center of the control box is re-centered to the center of the droplet at every $1.0 \mathrm{~s}$.

Column (a) and column (b) of Figure 3.4 show the results for the reduced and the 

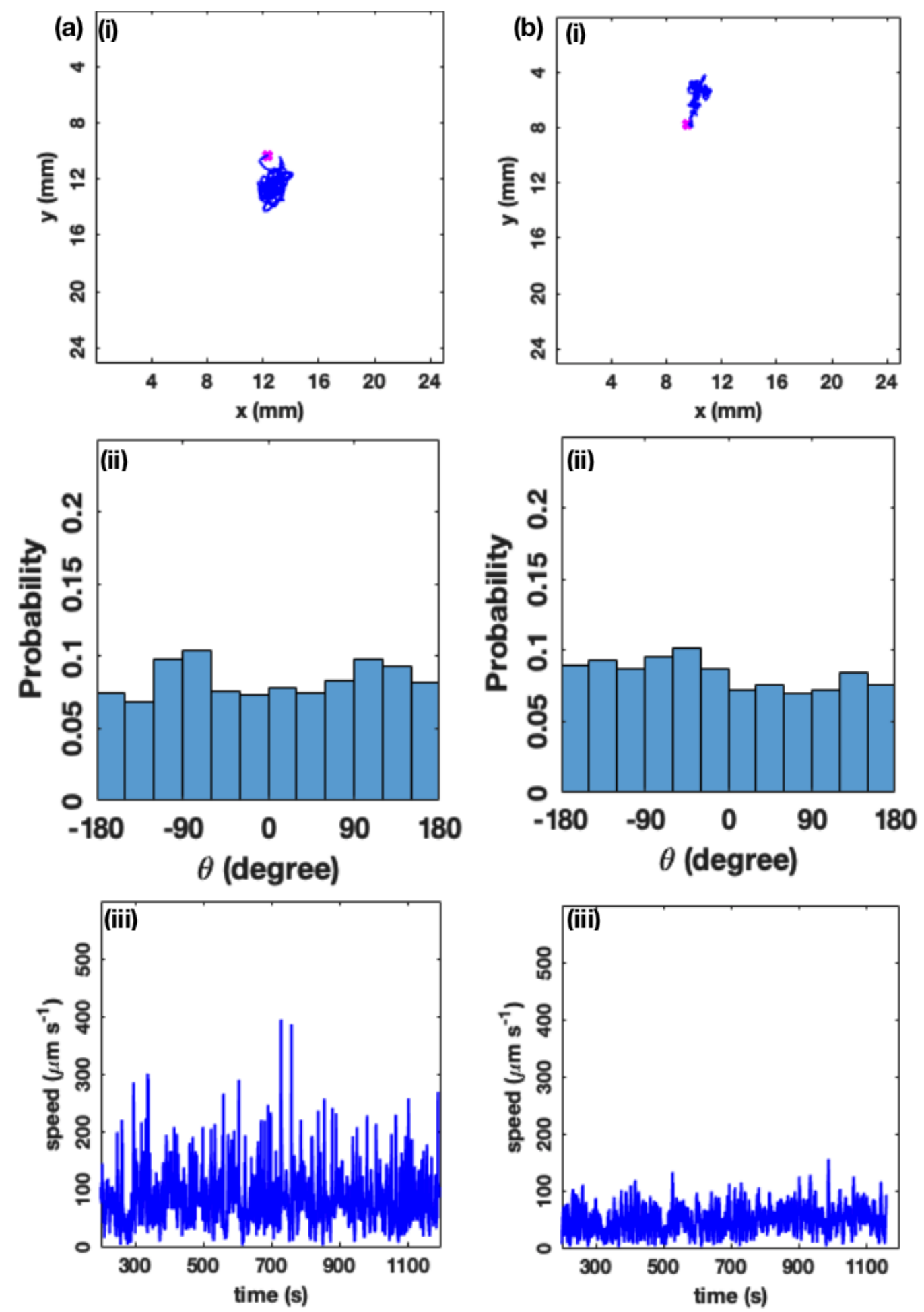

Figure 3.2: Results from the homogenous light intensity experiments. (a) Experiment with the reduced steady-state BZ droplet, $\left[\mathrm{Ru}(\mathrm{bpy})_{3}^{2+}\right]=1.88 \mathrm{mM}$, (i) droplet trajectory (ii) $\theta$ distribution (iii) droplet speed. (b) Experiment with the oxidized steady-state BZ droplet, $\left[\mathrm{Ru}^{2+}\right]=1.25 \mathrm{mM}$, (i) droplet trajectory (ii) $\theta$ distribution (iii) droplet speed. Other experimental conditions, $[\mathrm{MA}]=0.2 \mathrm{M},\left[\mathrm{NaBrO}_{3}\right]=0.3 \mathrm{M},\left[\mathrm{H}_{2} \mathrm{SO}_{4}\right]=0.6 \mathrm{M},[\mathrm{NaBr}]=0.03 \mathrm{M}$, and $[$ monoolein $]=10 \mathrm{mM}$. Image taken from reference [10]. 
oxidized steady-state BZ droplet under a constant light intensity gradient, respectively. The reduced steady-state BZ droplet is more responsive to the imposed light intensity gradient compared to its oxidized steady-state counterpart. This is supported by the trajectory plots in Figure $3.4 \mathrm{a}(\mathrm{i})$ and b(i) for the reduced and the oxidized steady-state BZ droplet, respectively. The trajectory plot for the reduced steady-state BZ droplet shows that the droplet moves from left to right. This direction corresponds to the droplet moving down the light intensity gradient. The angle $\theta$ distribution for the reduced steady-state BZ droplet shows that the $\mathrm{BZ}$ droplet direction occurs mostly around $-60^{\circ}$ to $-30^{\circ}$ range. These values correspond to an upward direction and to the right, which are depicted in the trajectory plot of the reduced steady-state BZ droplet.

The trajectory plot for the oxidized steady-state BZ droplet shows that the droplet undergoes a small displacement to the right. However, the displacement of the oxidized steady-state BZ droplet is not as significant as its reduced steady-state counterpart since the oxidized steady-state BZ droplet only moves approximately one diameter away from its original position. This indicates the oxidized steady-state BZ droplet is much less responsive toward the imposed light intensity gradient compared to its reduced steady-state counterpart. The angle $\theta$ distribution for the oxidized steady-state BZ droplet is relatively flat with a slight increase around $-90^{\circ}$ and $90^{\circ}$. This shows that the oxidized steady-state BZ droplet has minimal response toward the imposed light intensity gradient. The average speed of the reduced steady-state BZ droplet is $82.2 \mu \mathrm{m} \mathrm{s} s^{-1}$, and it is significantly greater than the average speed of the oxidized steady-state BZ droplet, which is $51.5 \mu \mathrm{m} \mathrm{s} s^{-1}$.

Based on the results from this set of experiments, it is clear that better directional control can be achieved using a reduced steady-state BZ droplet. Therefore, a reduced steady-state BZ droplet is used in complex trajectory experiments in order to further demonstrate the ability to control the directionality of the BZ droplet using a light intensity 
gradient.

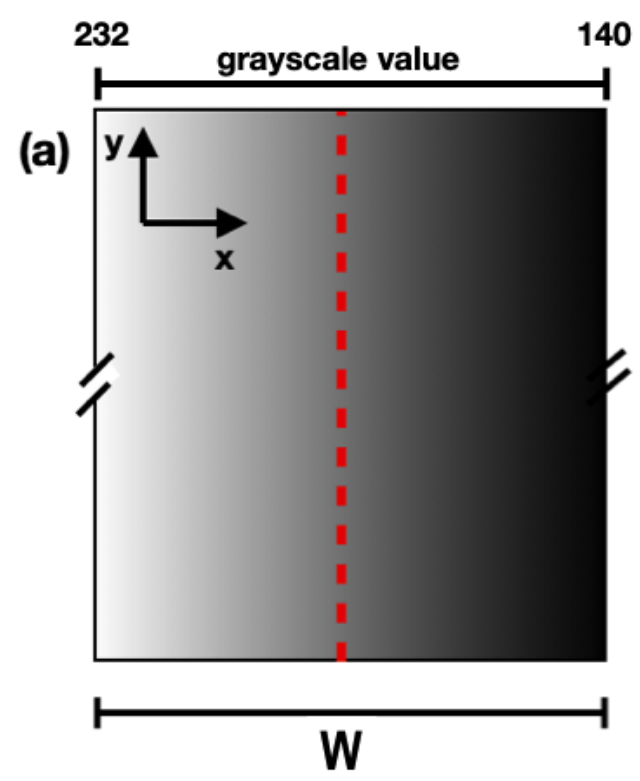

(b)

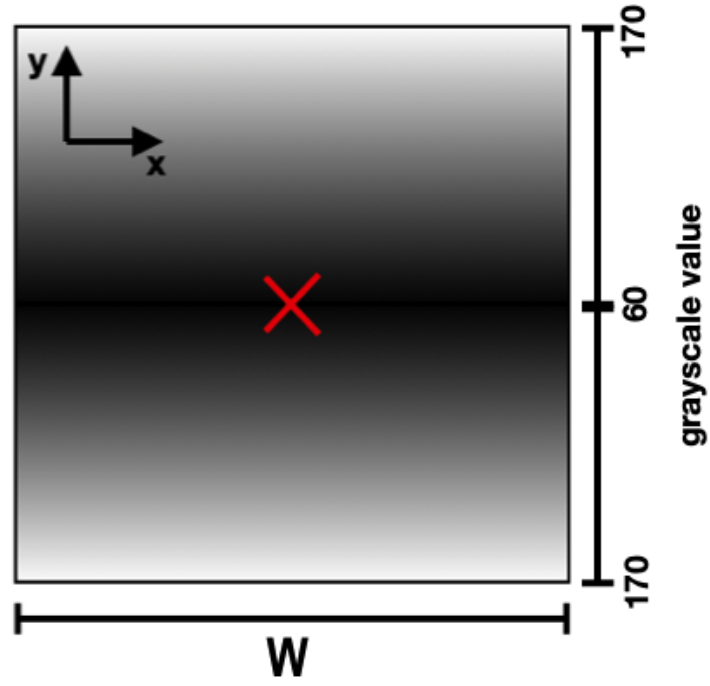

Figure 3.3: Light intensity gradient applied on the BZ droplet in (a) constant light intensity gradient experiments and (b) complex BZ droplet trajectory experiments. The dashed line and the cross in (a) and (b) are considered the center of the control box for each case. The grayscale values and its corresponding light intensities are as follows: (a) $232=7.376 \times 10^{-4}$ $\mathrm{W} \mathrm{cm} \mathrm{cm}^{-2}$ and $140=2.383 \times 10^{-4} \mathrm{~W} \mathrm{~cm}^{-2}$, (b) $170=3.684 \times 10^{-4} \mathrm{~W} \mathrm{~cm}^{-2}$ and $60=4.632$ $\times 10^{-5} \mathrm{~W} \mathrm{~cm}^{-2}$. The background grayscale value and its corresponding light intensity value is $255=9.090 \times 10^{-4} \mathrm{~W} \mathrm{~cm}^{-2}$. Image taken from reference [10].

\subsubsection{Complex BZ Droplet Trajectories}

To further demonstrate the ability in controlling the BZ droplet movements, complex trajectories are made using the reduced steady-state BZ droplet. The reduced steady-state BZ droplet is imposed with a light intensity gradient using a square control box that has a "V-shaped" form, as shown in Figure 3.3(b). The width of the box is set to be $W=0.18 \mathrm{~cm}$. By assuming that the BZ droplet center is located at $\left(x_{0}, y_{0}\right)$, the illumination intensity of the control box can be determined by the following: $\phi(x, y)=c_{1}\left|x-x_{0}\right|+c_{0}$, for $\left|y-y_{0}\right| \leqslant W / 2$ and $\left|x-x_{0}\right| \leqslant W / 2$, where $\phi(x, y)$ is the local illumination intensity within the control box, $c_{0}=4.63 \times 10^{-5} W \mathrm{~cm}^{-2}$, and $c_{1}=3.57 \times 10^{-3} \mathrm{~W} \mathrm{~cm}^{-3}$. Outside of the control box, the 

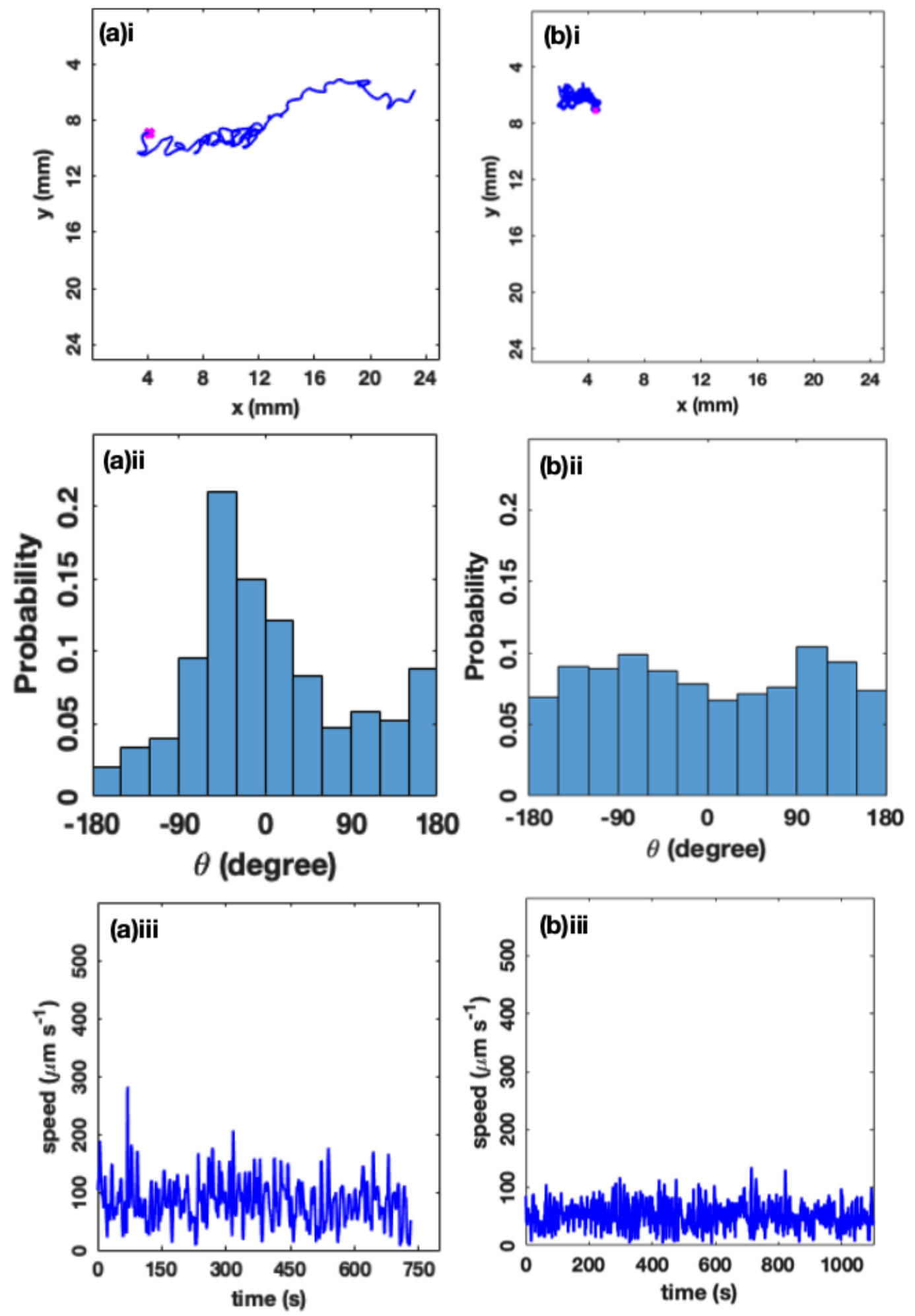

Figure 3.4: Results from one-dimensional BZ droplet direction control experiments.(a) Experiment with reduced steady-state BZ droplet, $\left[\mathrm{Ru}(\text { bpy })_{3}^{2+}\right]=1.88 \mathrm{mM}$ (b) experiment with oxidized steady-state $\mathrm{BZ}$ droplet, $\left[\mathrm{Ru}^{2+}\right]=1.25 \mathrm{mM}$. Other experimental conditions, $[\mathrm{MA}]$ $=0.2 \mathrm{M},\left[\mathrm{NaBrO}_{3}\right]=0.3 \mathrm{M},\left[\mathrm{H}_{2} \mathrm{SO}_{4}\right]=0.6 \mathrm{M},[\mathrm{NaBr}]=0.03 \mathrm{M}$, and $[$ monoolein $]=10 \mathrm{mM}$. Image taken from reference [10]. 
illumination is maintained at $9.09 \times 10^{-4} W \mathrm{~cm}^{-2}$. To move the BZ droplet in any direction, the control box is rotated so that it is parallel to the desired direction.

Other shapes of the light intensity gradient have also been utilized, but they result in a less precise control. For example, when a radially symmetric light intensity gradient is used, the BZ droplet tends to "orbit" around the center of the control box. With the "V-shaped" light intensity gradient box, the light intensity gradient functions to maintain the BZ droplet along the targeted trajectory. The discontinuity with the high light intensity at the trailing end of the BZ droplet helps to propel the BZ droplet forward.

In practice, the BZ droplet is controlled by moving the control box along the targeted trajectory. The control box is rotated so that it is tangent to the curve of the targeted trajectory. An image of the BZ droplet is taken every $1.0 \mathrm{~s}$. At each timestep, the distance between the BZ droplet center and the control box center is evaluated. If the distance is less than $0.9 \mathrm{~mm}$, the control box is moved a small distance along the targeted trajectory. The distance that the control box is moved ranges from $0.064 \mathrm{~mm}$ to $0.137 \mathrm{~mm}$ depending on the shape of the targeted trajectory. If the distance is greater than $0.9 \mathrm{~mm}$, the control box stays at the same location for that timestep.

There are a total of six complex trajectories that are used in this set of experiments. The complex trajectories are a circle, a 3-lobe hypotrochoid, a 4-lobe hypotrochoid, a modified hypotrochoid 1 , a modified hypotrochoid 2 , and a modified hypotrochoid 3 . The trajectories for the circle, the 3-lobe hypotrochoid, and the 4-lobe hypotrochoid can be generated using the following parametric equations:

$$
\begin{aligned}
& x=(\alpha-\beta) \cos (t)+\gamma \cos \left[\left(\frac{\alpha}{\beta}-1\right) t\right]+x_{0}, \\
& y=(\alpha-\beta) \sin (t)-\gamma \sin \left[\left(\frac{\alpha}{\beta}-1\right) t\right]+y_{0},
\end{aligned}
$$

where $\alpha, \beta$, and $\gamma$ are adjustable parameters that allow for the generation of the different target trajectories, and $t$ is the timestep. The following parameters are used for the circle 
trajectory: $\alpha=0, \beta=225$, and $\gamma=0$. For the 3-lobe hypotrochoid trajectory, the following parameters are used: $\alpha=90, \beta=30$, and $\gamma=120$. The 4-lobe trajectory requires the following parameter values: $\alpha=90, \beta=22.5$, and $\gamma=120$. The modified hypotrochoid 1 , 2, and 3 can be generated by using the following parametric equations:

$$
\begin{aligned}
& x=(\alpha+\varepsilon \beta) \cos (t)+\eta \gamma \cos \left[\left(\frac{\alpha}{\beta}-1\right) t\right]+x_{0}, \\
& y=(\alpha+\varepsilon \beta) \sin (t)+\eta \gamma \sin \left[\left(\frac{\alpha}{\beta}-1\right) t\right]+y_{0} .
\end{aligned}
$$

For the modified hypotrochoid 1 , the parameter values are $\varepsilon=-1, \eta=1, \alpha=125, \beta=25$, and $\gamma=125$. The parameter values for the modified hypotrochoid 2 are $\varepsilon=1, \eta=1$, $\alpha=125, \beta=25$, and $\gamma=125$. As for the modified hypotrochoid 3 , the parameter values used are $\varepsilon=1, \eta=-1, \alpha=125, \beta=25$, and $\gamma=125$.

Figure 3.5 shows the results from this set of experiments. Column (i) of Figure 3.5 shows the BZ droplet trajectory plot for each targeted trajectory shape. In each trajectory plot, the dashed red line is the targeted trajectory while the solid blue line is the trajectory made by the BZ droplets. In all cases, the trajectories made by the BZ droplets closely match the targeted trajectories. The instantaneous velocity direction of the BZ droplets is again characterized using the angle $\theta$ distributions, and these are shown in column (ii) of

Figure 3.5. For this set of experiments, the angle $\theta$ is redefined so that the instantaneous velocity direction is defined with respect to the instantaneous targeted trajectory direction. Therefore, when $\theta \approx 0^{\circ}$, the BZ droplet is considered to closely follow the targeted trajectory. In all cases, the angle $\theta$ distributions are heavily concentrated around $0^{\circ}$, which signifies that the BZ droplet trajectory closely matches its targeted trajectory.

\subsubsection{BZ Droplet Trajectories as a Function of Time}

To study the effect of time progression on the BZ droplet behavior, the BZ droplet is allowed to complete a 3-lobe hypotrochoid trajectory for three times. Figure 3.6 shows the 

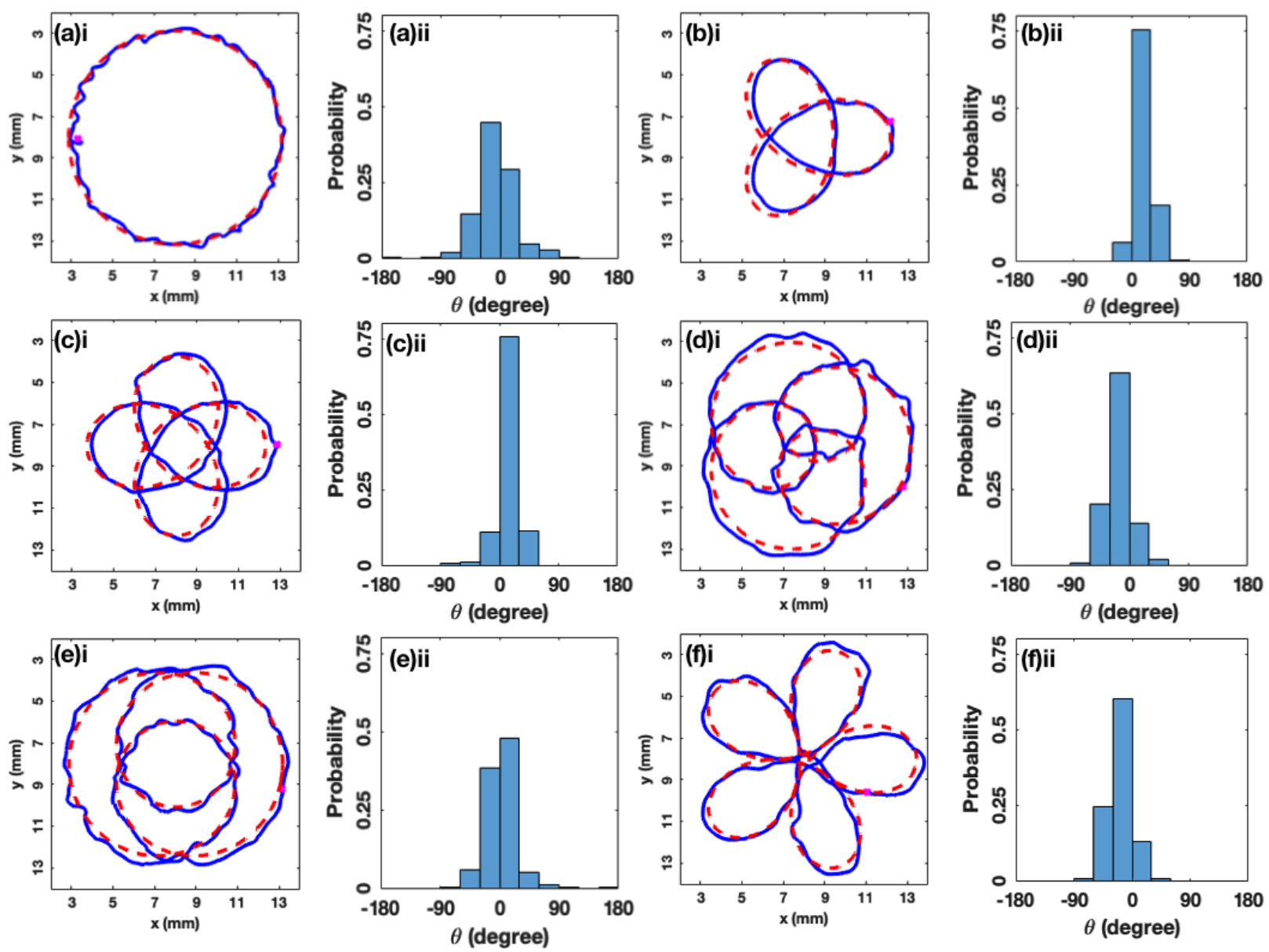

Figure 3.5: Different BZ droplet trajectories that are produced by modifying the parametric equations 3.1 and 3.2. (a) Circle, (b) 3-lobe hypotrochoid, (c) 4-lobe hypotrochoid, (d)-(f) modified hypotrochoids 1,2 , and 3 , respectively. In each subplot, the actual trajectory followed by the droplet is shown, blue, along with the target trajectory, dotted red line, generated by the respective parametric equations. Immediately adjacent to each subplot is the angle $\theta$ distribution. For all experiments, the following BZ recipe is used: $\left[\mathrm{NaBrO}_{3}\right]=$ $0.3 \mathrm{M},[\mathrm{MA}]=0.154 \mathrm{M},[\mathrm{BrMA}]=0.046 \mathrm{M},\left[\mathrm{H}_{2} \mathrm{SO}_{4}\right]=0.6 \mathrm{M},[\mathrm{NaBr}]=0.03 \mathrm{M},\left[\mathrm{Ru}(\mathrm{bpy})_{3}^{2+}\right]$ $=1.88 \mathrm{mM}$, and [monoolein $]=10 \mathrm{mM}$. Image taken from reference [10]. 
results from this set of experiments. The BZ droplet trajectory closely matches the targeted trajectory, and this is reflected in the angle $\theta$ distribution where the distribution is highly concentrated around $0^{\circ}$.

The distance traveled by the BZ droplet for each lap is $33.8 \mathrm{~mm}$, making the total distance covered by the BZ droplet $10.14 \mathrm{~cm}$. However, the BZ droplet takes $78 \mathrm{~s}$ longer to complete each additional lap. It takes the BZ droplet 1014 s, $1092 \mathrm{~s}$, and $1170 \mathrm{~s}$ to complete the first, second, and third lap, respectively. The increase in time for each additional lap completion can be attributed to the reactant consumption for the BZ droplet propulsion.

\subsection{Discussion}

Several studies have focused on using Marangoni flow to explain the propulsion mechanism. $\frac{59|11| 12}{5}$ The propulsion mechanisms from these studies are adapted to explain the propulsion mechanism for this project. After the creation of the BZ droplet, the surface of the BZ droplet is covered with fresh monoolein. The fresh monoolein lowers the surface tension of the BZ droplet surface. However, at the same time, the BZ reaction within the droplet generates bromine molecules as a result of its elementary reactions. The bromine molecules then diffuse into the BZ droplet surface and brominate the monoolein double bond. The bromination reaction causes the monoolein to be a less effective surfactant since its surface tension increases. Since the bromination reaction occurs stochastically, there are regions with a low surface tension because they are predominantly covered with the fresh monoolein, while some regions with a higher surface tension because they are predominantly covered with brominated monoolein. The difference in the surface tension results in fluid flow from low surface tension regions to higher surface tension regions on the BZ droplet surface. The fluid flow on the droplet surface causes the fluid inside and outside of the BZ 

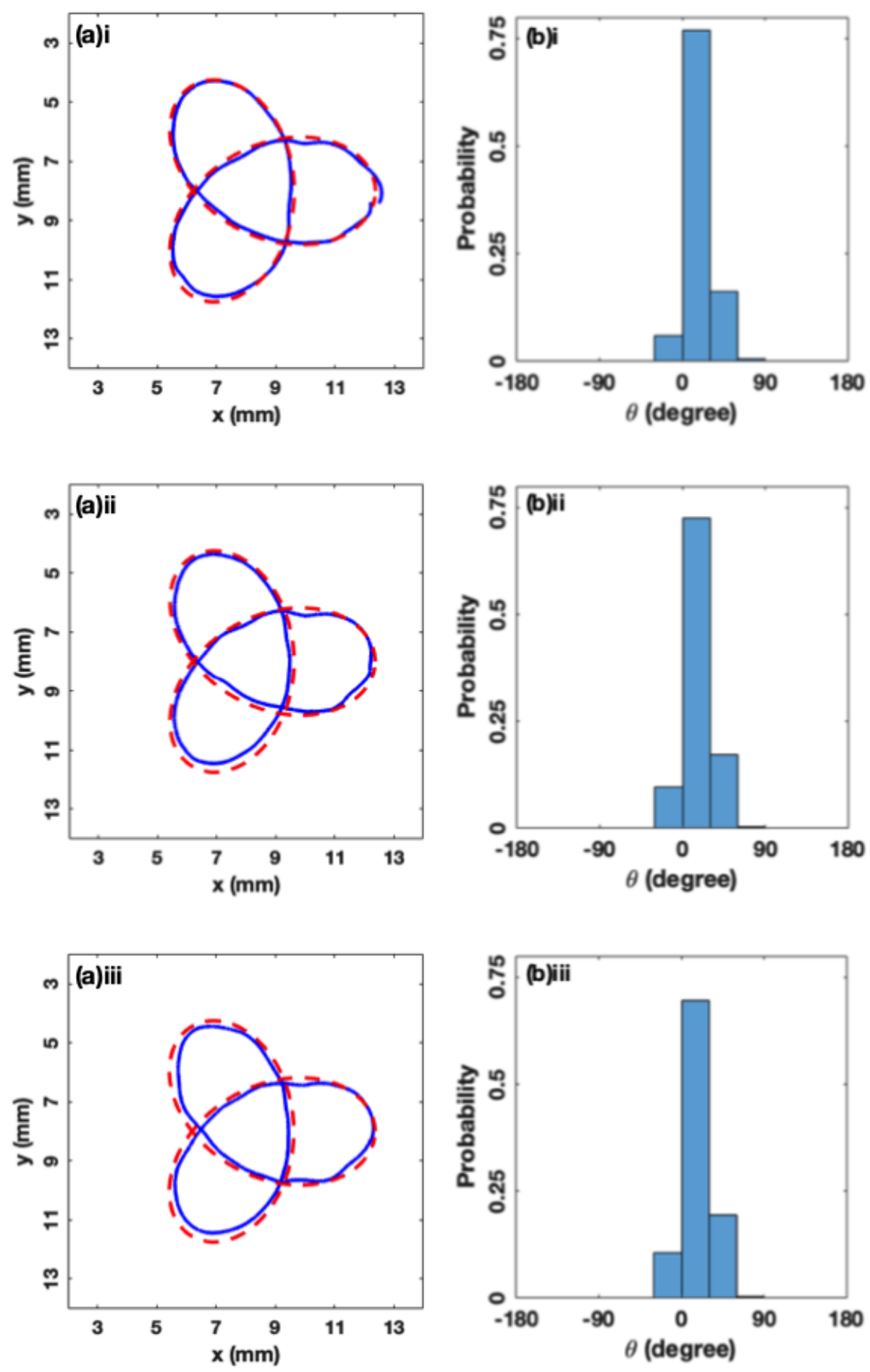

Figure 3.6: The BZ droplet trajectory for 3 laps of a 3-lobe hypotrochoid. (a) First, (b) second, and (c) third lap of the BZ droplet trajectory. The histogram to the right of each lap shows the corresponding angle $\theta$ distribution. Experimental conditions as in Figure 3.5 . Image taken from reference [10]. 
droplet to flow in the same direction due to the shearing stress. Due to the conservation of momentum, the BZ droplet moves in the opposite direction of the fluid flow.

In the case of the homogenous light intensity experiment, the droplet is exposed to homogenous light intensity. Therefore, any fluctuation in the bromination rate of the surfactant can be attributed to the stochastic process of the BZ reaction. This stochastic bromination reaction causes random regions on the BZ droplet to be brominated, which in turn causes the fluid flow to change direction. These flow changes are manifested in the random direction that the $\mathrm{BZ}$ droplet moves throughout the experiments.

In the case of experiments with the light intensity gradient, the BZ droplet movement is biased by affecting the BZ reaction within the droplet using a light intensity gradient. The light intensity affects the BZ reaction differently. There are two separate channels that the photosensitive BZ reaction can undergo. $\frac{15 \mid 16}{}$ Channel one is photoexcitatory, which involves the production of $\mathrm{HBrO}_{2}$ from $\mathrm{BrO}_{3}^{-}$species when the catalyst is exposed to a low light intensity while channel two involves the production of $\mathrm{Br}^{-}$species from $\mathrm{BrO}_{3}^{-}$species when the catalyst is exposed to a high light intensity.

The effect of different light intensities that the BZ droplet is exposed to can be simulated using a modified Oregonator-type model. Two new variables corresponding to $\mathrm{HBrO}_{2}$ and $\mathrm{Br}_{2}$ are added into the original model along with five extra steps involving the newly introduced variables. $\frac{5[17 \mid 18}{10}$ Table 3.1 shows a list of reactions with its respective rates. Photoexcitation of the reduced metal catalyst is represented by the reaction $L 0$. The excited metal catalyst then undergoes steps $L 1$ and L2, which represent the photoinhibitory and photoexcitatory channels, respectively. By assuming steady-state condition for species E, the excited form of the reduced metal catalyst, the rate for the two processes are $p_{1} \psi$ and $p_{2} \psi$, respectively. $\psi$ is the light intensity simulation while $p_{1}$ and $p_{2}$ can be determined as 


\begin{tabular}{|lll|}
\hline & reaction & rate \\
\hline$(1)$ & $\mathrm{A}+\mathrm{Y} \rightarrow \mathrm{X}+\mathrm{P}$ & $k_{1} A Y$ \\
$(2)$ & $\mathrm{P}+\mathrm{Y} \rightarrow \mathrm{U}$ & $k_{2} P Y$ \\
$(3)$ & $\mathrm{U} \rightarrow \mathrm{P}+\mathrm{Y}$ & $k_{3} U P$ \\
$(4)$ & $\mathrm{X}+\mathrm{Y} \rightarrow 2 \mathrm{P}$ & $k_{4} X Y$ \\
$(5)$ & $\mathrm{A}+\mathrm{X} \rightleftarrows 2 \mathrm{~W}$ & $k_{5} A X, k_{-5} W^{2}$ \\
$(6)$ & $\mathrm{P}+\mathrm{X} \rightarrow \mathrm{Y}$ & $k_{6} P X$ \\
$(7)$ & $2 \mathrm{X} \rightarrow \mathrm{P}+\mathrm{A}$ & $k_{7} X^{2}$ \\
$(8)$ & $\mathrm{G}+\mathrm{W} \rightarrow \mathrm{X}+\mathrm{Z}$ & $k_{8}\left(C_{0}-Z\right) W$ \\
$(9)$ & $\mathrm{Z} \rightarrow h \mathrm{Y}$ & $k_{9} Z$ \\
$(10)$ & $\mathrm{P}+\mathrm{B} \rightarrow \mathrm{V}$ & $k_{10} P B$ \\
$(11)$ & $\mathrm{V} \rightarrow \mathrm{products}$ & $k_{11} V$ \\
$(12)$ & $\mathrm{B}+\mathrm{U} \rightarrow \mathrm{V}+\mathrm{Y}$ & $k_{12} B U$ \\
$(13)$ & $\mathrm{U} \rightarrow$ & $k_{13}$ \\
$(\mathrm{~L} 0)$ & $\mathrm{G} \rightleftarrows \mathrm{E}$ & $\left(C_{0}-Z\right) \psi, k_{-L 0} E$ \\
$(\mathrm{~L} 1)$ & $\mathrm{E}+\mathrm{A}+\mathrm{H} \rightarrow \mathrm{Y}+\mathrm{Z}$ & $k_{L 1} E V H$ \\
$(\mathrm{~L} 2)$ & $\mathrm{E}+\mathrm{A}+2 \mathrm{H} \rightarrow \mathrm{W}+\mathrm{Z}$ & $k_{L 2} E A H^{2}$ \\
\hline
\end{tabular}

Table 3.1: Model variables $\mathrm{X}=\left[\mathrm{HBrO}_{2}\right], \mathrm{Y}=\left[\mathrm{Br}^{-}\right], \mathrm{Z}=\left[\mathrm{Ru}(\mathrm{bpy})_{3}^{3+}\right], \mathrm{W}=\left[\mathrm{BrO}_{2}.\right], \mathrm{V}=$ $\left[\mathrm{CHBr}(\mathrm{COOH})_{2}\right], \mathrm{P}=[\mathrm{HOBr}], \mathrm{E}=\left[\mathrm{Ru}(\mathrm{bpy})_{3}^{2+*}\right], \mathrm{G}=\left[\mathrm{Ru}(\mathrm{bpy})_{3}^{2+}\right]$ and $\mathrm{U}=\left[\mathrm{Br}_{2}\right]$. System parameters are given as $\mathrm{A}=\left[\mathrm{BrO}_{3}^{-}\right], \mathrm{H}=\left[\mathrm{H}^{+}\right]$, $\mathrm{h}$ is the stoichiometric factor, $\mathrm{C}_{0}$ is the total amount of ruthenium in the system, with $\mathrm{C}_{0} \approx \mathrm{Z}+\mathrm{G}$. $\mathrm{B}_{0}$ is a constant equal to the sum of the malonic acid and bromomalonic acid concentrations, with $\mathrm{B}=[\mathrm{MA}]=\mathrm{B}_{0}\left(1-\mathrm{V} / \mathrm{B}_{0}\right)$.

follows:

$$
p_{1}=\frac{H V\left(C_{0}-Z\right)}{\frac{k_{-L 0}}{k_{L 1}}+\frac{k_{L 2}}{k_{L 1}} H^{2} A}, \quad \quad p_{2}=\frac{\frac{k_{L 2}}{k_{L 1}} H^{2} A\left(C_{0}-Z\right)}{\frac{k_{-L 0}}{k_{L 1}}+H V+\frac{k_{L 2}}{k_{L 1}} H^{2} A} .
$$

Step 13 represents the loss of bromine through bromination reaction with the surfactant, monoolein, on the surface of the droplet, and through diffusion from the BZ droplet into the oil-phase. ${ }^{5}$ To study the effect of different light intensity exposed to the different oxidation state of the ruthenium catalyst, two different conditions are employed. The constant $h$ in reaction (9) is set to 0.45 and 0.55 for the reduced and the oxidized steady-state, respectively. 


\begin{tabular}{|c|c|c|}
\hline constant & value & ref \\
\hline$k_{1}$ & $2 H^{2}$ & 17 \\
\hline$k_{2}$ & $2.3 \times 10^{9} \mathrm{H}$ & 18 \\
\hline$k_{3}$ & 2 & 18 \\
\hline$k_{4}$ & $3 \times 10^{6} \mathrm{H}$ & 17 \\
\hline$k_{5}$ & $42 H$ & 17 \\
\hline$k_{-5}$ & $4.2 \times 10^{7}$ & 17 \\
\hline$k_{6}$ & 3.2 & 18 \\
\hline$k_{7}$ & 3000 & 17 \\
\hline$k_{8}$ & $8 \times 10^{6} \mathrm{H}$ & 17 \\
\hline$k_{9}$ & 1.1 & 17 \\
\hline$k_{10}$ & $8.2 B_{0}$ & 17 \\
\hline$k_{11}$ & $2 \times 10^{-2}$ & 17 \\
\hline$k_{12}$ & 40 & 18 \\
\hline$k_{13}$ & $5.2 \times 10^{-1}$ & 5 \\
\hline$\frac{k_{-L 0}}{k_{L 1}}$ & 0.0223 & 5 \\
\hline$\frac{k_{L 2}}{k_{L 1}}$ & 5.54 & 5 \\
\hline $\mathrm{A}$ & 0.3 & \\
\hline $\mathrm{B}_{0}$ & 0.2 & \\
\hline $\mathrm{C}_{0}$ & 0.0015 & \\
\hline $\mathrm{H}$ & 0.6 & \\
\hline $\mathrm{h}$ & 0.45 or 0.55 & \\
\hline$\psi$ & 0.1 or 0.2 & \\
\hline
\end{tabular}

Table 3.2: Rate constants and system parameters corresponding to the model given in Table 3.1 .

Figure 3.7 shows the result from the simulation. Figure 3.7(a) shows the amount of bromine produced by the BZ reaction under the reduced (blue line) and the oxidized (red) steady-state. When the BZ reaction is under the oxidized steady-state condition, an increase in light intensity increases the bromine production by a small amount $(<0.5 \%)$. On the other hand, when the BZ reaction is under the reduced steady-state condition, the production of bromine has a transient large increase in the bromine production followed by a steady output 

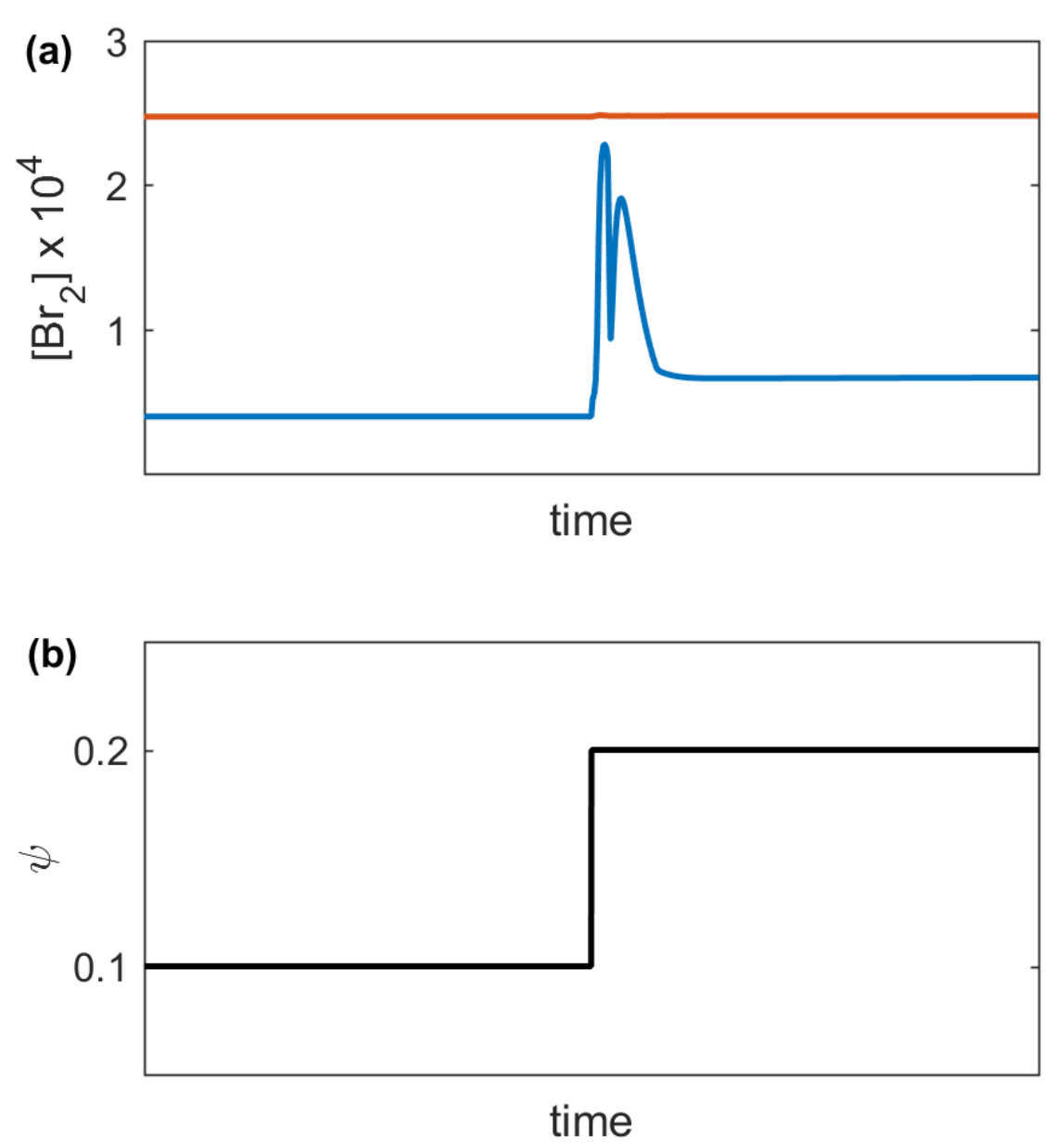

Figure 3.7: Simulation results using a modified Oregonator-type model. The reactions involved are listed in Table 3.1 while the variables used are listed in Table 3.2. (a) A plot of $\mathrm{Br}_{2}$ concentration as a function of dimensionless time for reduced (blue line) and oxidized (orange line) steady-state conditions. (b) A plot of light intensity as a function of dimensionless time. Images taken from reference [10].

of bromine, which is $60 \%$ larger than the initial output.

The different behaviors observed between the oxidized and the reduced steady-state BZ droplet can be explained using the results from the simulation. For the oxidized steadystate BZ droplet, the light intensity gradient applied affects the droplet minimally. This is because the amount of bromine generated under the different light intensity is small $(<0.5 \%)$. This results in only a small surface tension difference between regions under a low light 
intensity and regions under a high light intensity. The small surface tension difference leads to small Marangoni flow on the oxidize steady-state BZ droplet surface, which translates to a small displacement.

On the other hand, the reduced steady-state BZ droplet experienced a large difference in surface tension under the light intensity gradient. Figure 3.7(a) shows that the reduced steady-state BZ droplet produces more bromine under a high light intensity compared to when it is under a low light intensity. The large difference in the bromine production translates to a large difference in the bromination rate of the monoolein. Subsequently, this creates a large surface tension gradient on the surface of the droplet. Greater surface tension gradients in the reduced steady-state BZ droplet create greater flows of fluid from low surface tension regions to high surface tension regions. Thus, more momentum is transferred from the droplet to the surrounding fluid. This leads to a greater propulsion of the BZ droplet effectively moving down the light intensity gradient.

In the case of the complex trajectory experiments, the trailing end of the BZ droplet is constantly exposed to the high intensity light because of the discontinuity of the control box. The trailing edge of the reduced steady-state BZ droplet is continuously producing a high concentration of bromine molecules, whereas the leading edge is continuously producing a low concentration of bromine molecules. This bias and the fluid flow help maintain the surface tension gradient across the reduced steady-state BZ droplet, which leads to persistent motion of the droplet.

\subsection{Conclusion}

The results presented in this chapter show that the BZ droplet does not have a preferred direction under homogenous light intensity. The BZ droplet consistently changes 
direction due to the stochastic nature of the the bromination of the surfactant on the BZ droplet surface. This behavior is observed in the reduced and the oxidized steady-state BZ droplet and it is supported by the trajectory plots and the angle $\theta$ distributions.

Under a constant light intensity gradient, the reduced steady-state BZ droplet is more responsive than the oxidized steady-state BZ droplet. This is evident from the trajectory plot that shows the reduced steady-state BZ droplet moves from left to right, which is effectively moving down the light intensity gradient. The angle $\theta$ distribution also shows that there is a significant concentration of angle $\theta$ around $-60^{\circ}$ to $-30^{\circ}$, which shows that there is a preferred direction for the reduced steady-state BZ droplet. This behavior does not appear in the oxidized steady-state BZ droplet under the same condition.

Since the reduced steady-state BZ droplet shows significant response to the light intensity gradient, it is used in the complex trajectory experiments that show excellent motion control of the BZ droplet using a light intensity gradient. The trajectory plots and the angle $\theta$ distributions for the complex trajectories demonstrate the accuracy of the BZ droplet motion control using a light intensity gradient. 


\subsection{References}

[1] J. P. Keener and J. J. Tyson, Phys. D 21, 307 (1986).

[2] A. Winfree and W. Jahnke, J. Phys. Chem. 93, 2823 (1989).

[3] R. M. Noyes, R. J. Field, and E. Körös, J. Am. Chem. Soc. 94, 1394 (1971).

[4] R. J. Field and R. M. Noyes, J. Chem. Phys. 60, 1877 (1974).

[5] N. J. Suematsu, Y. Mori, T. Amemiya, and S. Nakata, J. Phys. Chem. Lett. 7, 3424 (2016).

[6] S. Kitawaki, K. Shioiri, T. Sakurai, and H. Kitahata, J. Phys. Chem. C 116, 26805 (2012).

[7] M. L. Smith, K. Heitfield, M. Tchoul, and R. A. Vaia, Proc. SPIE , 79750A (2011).

[8] H. Kitahata, N. Yoshinaga, K. H. Nagai, and Y. Sumino, Chem. Lett. 41, 1052 (2012).

[9] S.Thutupalli, Towards Autonomous Soft Matter Systems, Ph.D. thesis, University of Göttingen (2014).

[10] S. J. S. Jamaluddin, K. Khaothong, M. R. Tinsley, and K. Showalter, Chaos 30, 083143 (2020).

[11] S. Thutupalli, R. Seeman, and S. Herminghaus, New J. Phys. 13, 073021 (2011).

[12] S. Herminghaus, C. C. Maass, C. Krüger, S. Thutupalli, L. Goehring, and C. Bahr, Soft Matter 10, 7008 (2014).

[13] A. Diguet, R.-M. Guillermic, N. Magome, A. Saint-Jalmes, Y. Chen, K. Yoshikawa, and D. Baigl, Angew. Chem., Int. Ed. 48, 9281 (2009). 
[14] K. Kalyanasundaram, Coord. Chem. Rev. 46, 159 (1982).

[15] S. Kádár, T. Amemiya, and K. Showalter, J. Phys. Chem. A 101, 8200 (1997).

[16] I. Hanazaki and Y. Mori and T. Sekiguchi and G. Rábai, Phys. D 84, 228 (1995).

[17] T. Amemiya and T. Yamamoto and T. Ohmori and T. Yamaguchi, J. Phys. Chem. A 106, $612(2002)$.

[18] L. Györgyi and R. J. Field, J. Phys. Chem. 95, 6594-6602 (1991). 


\section{Chapter 4}

\section{Propagating Aluminum Hydroxide}

\section{Precipitate under pH Field Influence}

\subsection{Introduction}

Self-organized patterns have been observed in nature for a long time. These include patterns made by lichen growth, pigmentation on a porphyry olive shell, and patterns on marbled cone shells. ${ }^{11}$ Scientists have been trying to replicate and study these patterns in the laboratory. Candidates for self-organized pattern forming systems include the BelousovZhabotinsky (BZ) reaction, the Liesegang system, and the precipitation/redissolution system such aluminum hydroxide systems. ${ }^{2 / 4}$

Patterns in the BZ systems typically form in a homogeneous system. It is typically produced by thoroughly mixing sodium bromate, sulfuric acid, malonic acid, sodium bromide, and a metal catalyst. The solution is then poured into a petri dish to form a thin layer of the BZ solution. The thin layer BZ solution typically develops spatial patterns if the solution is left unstirred. Some of the patterns that have been documented to form in this system include target patterns, spiral waves patterns, counterrotating spiral patterns, 
and traveling waves. $\frac{215,8}{8}$

Unlike the BZ reaction, the Liesegang patterns form in a heterogeneous system. The Liesegang patterns are typically produced by the precipitation reaction between two inorganic salt solutions. One of the salt solutions, called the inner electrolyte, is trapped in a gel matrix such as agarose gel. The other inorganic salt solution, called the outer electrolyte, is typically poured on top of the gel containing the inner electrolyte or in a well created by carving out a portion of the gel matrix. The outer electrolyte is allowed to diffuse into the gel and react with the inner electrolyte to produce precipitate. The interplay of the precipitation reaction and the diffusivity of the electrolytes is responsible for the formation of the Liesegang pattern.

If the experiment is set up in a cylindrical tube with the outer electrolyte poured on top of the inner electrolyte, the pattern typically takes a form of periodic precipitation bands. For example, when lead(II) nitrate is immobilized in a gel matrix and potassium iodide solution is poured on top of the gel, alternating bands of yellow and colorless regions form. The colorless regions mark the absence of lead iodide precipitate while the yellow bands mark the presence of the lead iodide precipitate. ${ }^{9}$ These bands are stationary since they do not propagate after they have formed. Propagating Liesegang systems can be produced if the combination of the electrolytes allows precipitate to form and redissolve through complex ion formation with the excess outer electrolytes. An example for such combination is cobalt chloride (inner electrolyte) and ammonia (outer electrolyte). The precipitate that forms initially is cobalt hydroxide, $\mathrm{Co}(\mathrm{OH})_{2}$. With the presence of excess ammonia, the $\mathrm{Co}(\mathrm{OH})_{2}$ is converted to hexaamminecobalt(II) ions, $\mathrm{Co}\left(\mathrm{NH}_{3}\right)_{6}^{2+} . \underline{10}$

In 2007, Volford et al. showed the existence of self-organized patterns in precipitation system that resembles the self-organized patterns in the BZ reaction. This observation was considered a breakthrough since it was the first time such patterns were observed in 
a precipitation system. This system consists of aluminum chloride as the inner electrolyte and sodium hydroxide as the outer electrolyte. This system has been shown to produce patterns such as spiral waves patterns, counterrotating spiral patterns, and traveling waves. $4 \mid 11$ The behavior of the spatiotemporal patterns can be separated into two components. The first component involves the migrating precipitation band that moves downward from the gel/sodium hydroxide interface. This band is approximately $10-200 \mu \mathrm{m}$ in thickness. The thickness varies as a function of time and the concentration of the inner electrolyte. ${ }^{1112}$ The second component is the self-organized patterns that occur within the migrating band.

Cross-sectional experiments involving these spatiotemporal patterns show that the patterns are complex. These experiments showed that the traveling waves can be characterized by a diagonal feature. The diagonal feature connects the wavefront to the waveback of the propagating wave within the migrating band. Experiments and simulations suggest that the diagonal feature is made up of a different type of aluminum hydroxide crystal compared to the migrating band. $\frac{1113}{113}$ The crystal that make up the diagonal feature is only generated at the wavefront. As the migrating band moves downward, the wavefront moves sideways in addition to moving downward together with the migrating band. This makes the diagonal feature elongate. The traveling precipitation waves have also been shown to exist in other systems with electrolytes that can produce complexes, which includes zinc hydroxide and mercury iodide. $\frac{12[14 \mid 15}{}$ However, no extensive study has been conducted on these systems so far.

A variant of Volford et et. system includes an open aluminum hydroxide system. In this system, no electrolyte is dissolved in the gel. Instead, the gel is used to separate the sodium hydroxide and aluminum chloride solution. The electrolytes are then allowed to diffuse and interact within the gel to produce patterns. The open system has been shown to have additional behaviors that do not appear in closed systems. At low concentration of 
aluminum chloride, there are transient oscillations of the transmitted light intensity while at higher concentration of aluminum chloride, some cellular structures can develop. ${ }^{16}$ The speed of the waves has also been shown to increase from $0.22 \mathrm{~mm} \mathrm{~min}^{-1}$ to $0.27 \mathrm{~mm} \mathrm{~min}^{-1}$ as the concentration of the aluminum chloride is increased. In this study, a small amount of sulfuric acid $(0.01 \mathrm{M})$ is added into the aluminum chloride reservoir. However, no explanation was given for the addition of the sulfuric acid in this system.

The addition of the sulfuric acid in the open system of the aluminum hydroxide system could have been an attempt to create a $\mathrm{pH}$ field across the gel slab. $\mathrm{pH}$ is known to affect the aluminum hydroxide crystal formation. The aluminum hydroxide crystal can only exist within $\sim 4$ to $9.5 \mathrm{pH}$ range. ${ }^{[17}$ If the $\mathrm{pH}$ value is less than $\sim 4$, the aluminum species exists as free aluminum ions, $A l^{3+}$, whereas if the $\mathrm{pH}$ is greater than 9.5 , the aluminum species exists as aluminum hydroxide complex ions, $\mathrm{Al}(\mathrm{OH})_{4}^{-}$. However, solid aluminum hydroxide, $\mathrm{Al}(\mathrm{OH})_{3}$, is most prevalent within the $\mathrm{pH}$ range of $5-8.17+19$

In this chapter, the effect of the $\mathrm{pH}$ field on the behavior of the migrating precipitation band and the patterns formed is studied. The $\mathrm{pH}$ field is created and maintained with the addition of hydrochloric acid into the aluminum hydroxide system.

\subsection{Experimental Method}

Figure 4.1 shows the setup used in this study. The experimental setup consists of a light box, a chemical reactor, and a camera. The light box is placed behind the chemical reactor, and it acts as a source of illumination. All of the images taken for this study are backlit unless specified otherwise. The chemical reactor is made up of two large reservoirs separated by a middle section that is filled with a $1 \%$ agarose gel. The camera, which is connected to a computer, is used to record any patterns that formed in the gel as a result of 
the reaction occurring within the gel. The images are taken every 30 seconds.

The agarose gel is prepared by mixing the appropriate amount of agarose powder with water to make a $1 \% \mathrm{w} / \mathrm{w}$ agarose gel. The mixture is continuously stirred, and it is also heated to $90^{\circ} \mathrm{C}$ for 10 minutes. The gel mixture is then stirred for additional 10 minutes without heat. The gel mixture is then transferred into the middle section of the chemical reactor to solidify at room temperature. The two reservoirs of the chemical reactor are filled with different solutions. One reservoir is filled with a sodium hydroxide $(\mathrm{NaOH})$ solution while the other reservoir is filled with a mixture of aluminum chloride $\left(\mathrm{AlCl}_{3}\right)$ and hydrochloric acid $(\mathrm{HCl})$ solutions. The experiment begins once both reservoirs have been filled to the top. The solution in each reservoir is continuously stirred throughout the experiment.

The design of the chemical reactor used is different depending on the experiment. Figure 4.1(b) shows the schematic diagram of the reactor used in side-view experiment whereas Figure 4.1(c) shows the schematic diagram of the reactor used in front-view experiment. The side-view experimental setup allows for the observation of the migrating precipitation wave band while the top-view experimental setup allows for the observation of the propagating precipitation waves.

\subsection{Results}

\subsubsection{Side-view Experiment}

Since the reactor is backlit, the presence of aluminum hydroxide, $\mathrm{Al}(\mathrm{OH})_{3}$, precipitate blocks the light from transmitting through the gel. As a result, regions with high concentration of $\mathrm{Al}(\mathrm{OH})_{3}$ precipitate appear darker than the surrounding regions. Figure 4.2 (a) shows a snapshot of the gel from one of the experiments conducted using the side-view 


\section{Camera}

(a)
Light source
Reactor
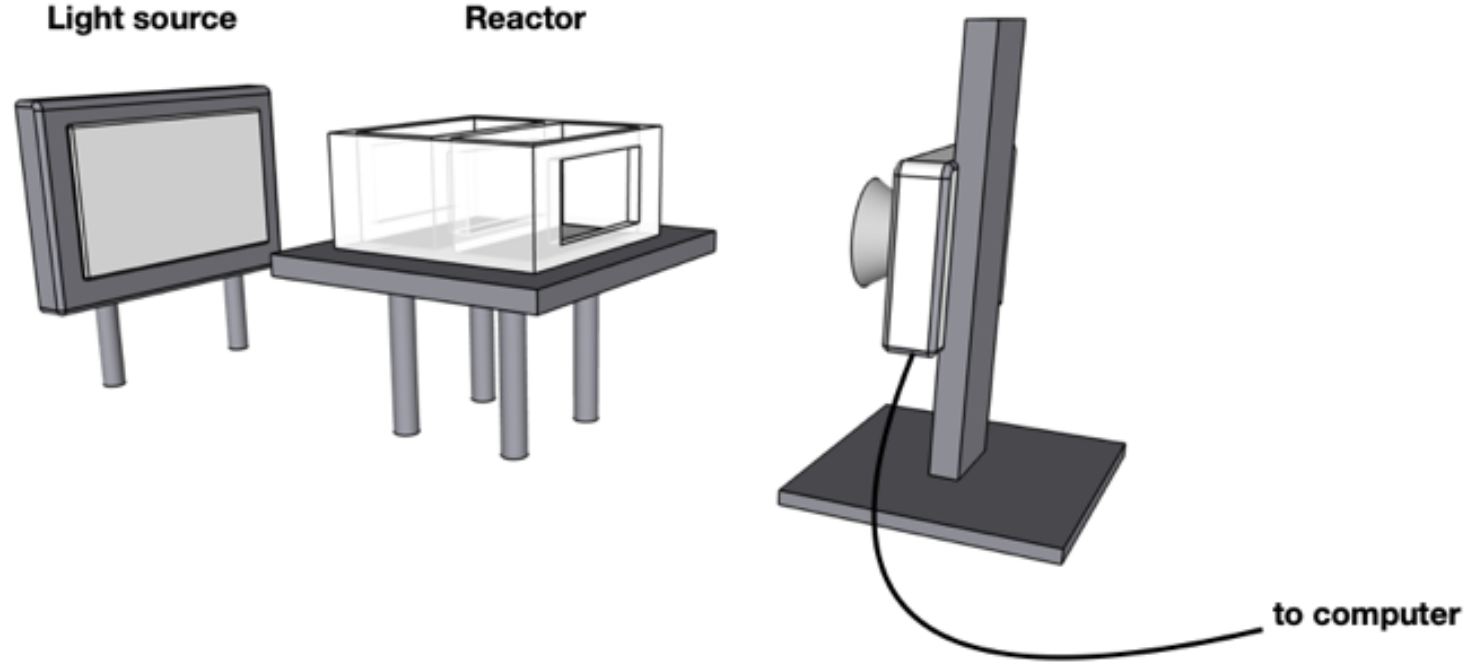

(b)

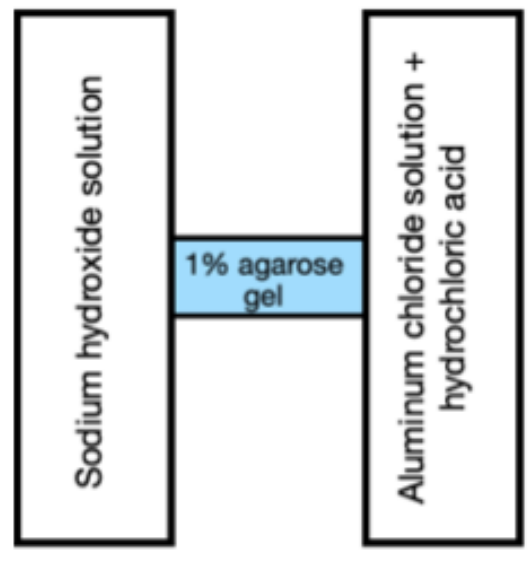

(2)

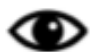

Observation (c)

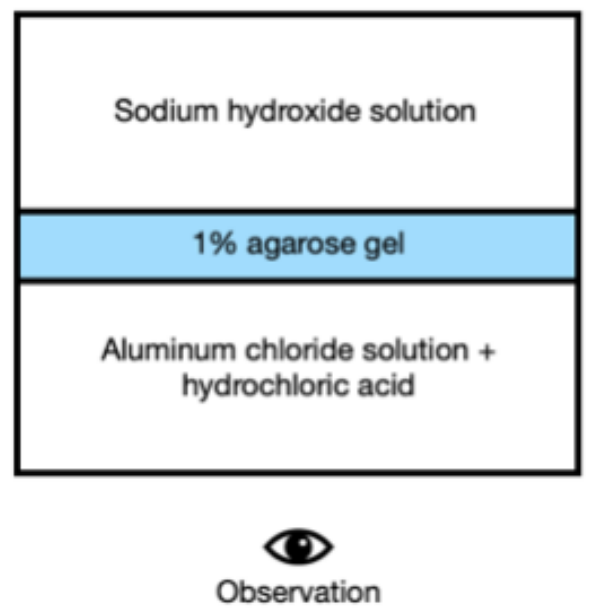

Figure 4.1: Experimental setup used in this study. (a) The overall setup used in this study. The reactor used in the experiment is placed in between the camera and the light source. Unless noted, all of the experimental results presented here are backlit. A schematic drawing of the reactor used for (b) side-view experiment and (c) front-view experiment.

chemical reactor. The thin black line in the middle of the image is the migrating $\mathrm{Al}(\mathrm{OH})_{3}$ precipitate band. The band migrates from the $\mathrm{NaOH}$ reservoir toward the $\mathrm{AlCl}_{3} / \mathrm{HCl}$ reservoir. The blue and yellow coloration of the gel is caused by the addition of bromothymol blue, a pH indicator, into the gel. The bromothymol blue allows for the determination and 

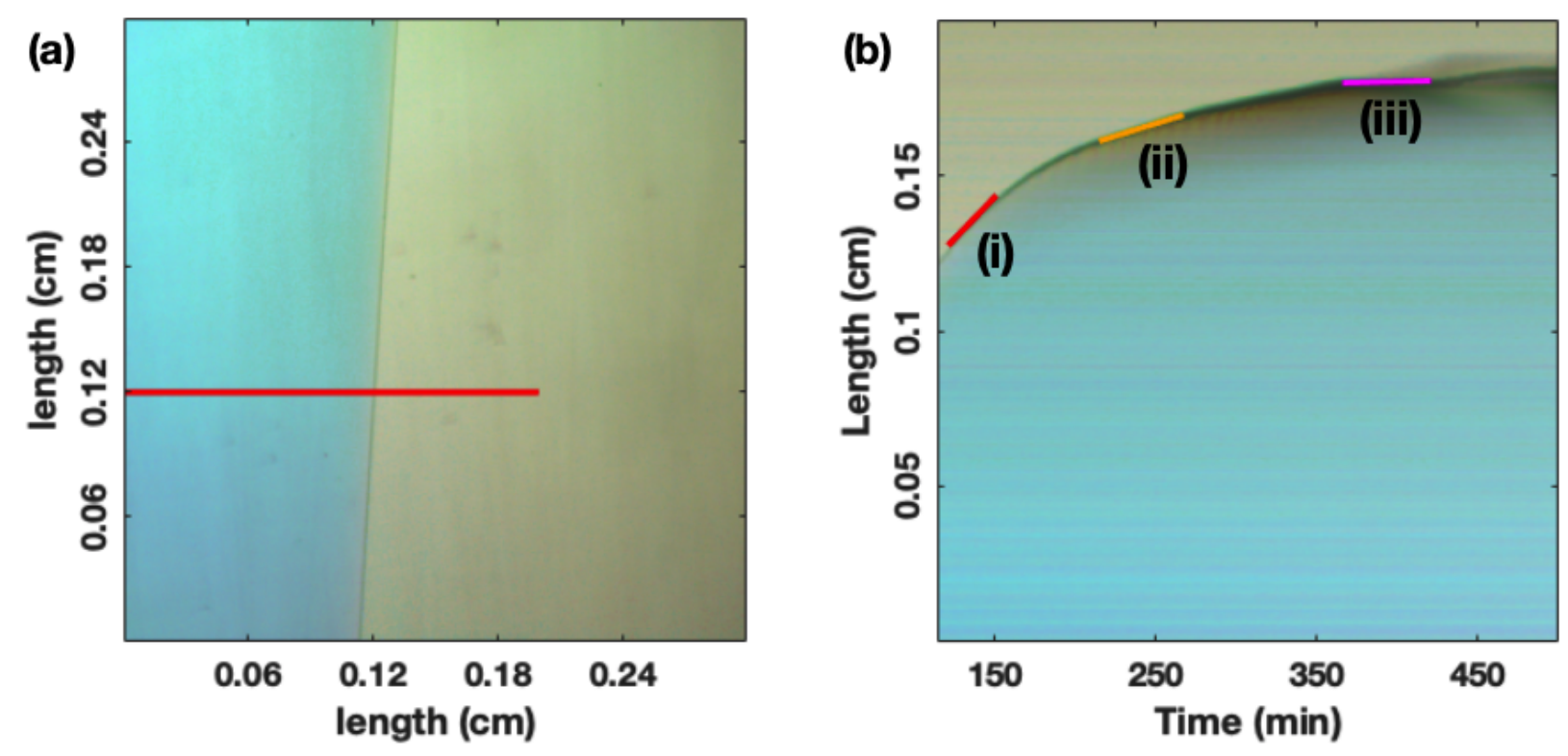

Figure 4.2: (a) A snapshot of the side-view experimental result. The red line indicates the slice used for constructing the spacetime plot. (b) A spacetime plot constructed from experiment in (a). The slope of the migrating band in the spacetime plot gives the speed of the migrating band: (i) $5.39 \times 10^{-4} \mathrm{~cm} \mathrm{~min}^{-1}$, (ii) $1.60 \times 10^{-5} \mathrm{~cm} \mathrm{~min}^{-1}$, and $1.18 \times$ $10^{-5} \mathrm{~cm} \mathrm{~min}^{-1}$. The experimental conditions are $\left[\mathrm{AlCl}_{3}\right]_{0}=0.16 \mathrm{M},[\mathrm{HCl}]_{0}=0.06 \mathrm{M}$, $[\mathrm{NaOH}]_{0}=0.48 \mathrm{M}$, and $[$ agarose $]=1.0 \% \mathrm{w} / \mathrm{w}$.

visualization of the gel $\mathrm{pH}$ while conducting the experiment. The blue color indicates that the region to the left of the $\mathrm{Al}(\mathrm{OH})_{3}$ precipitate band is basic $(\mathrm{pH}>7.6)$ while the yellow color to the right of the $\mathrm{Al}(\mathrm{OH})_{3}$ precipitate band indicates that the region is acidic $(\mathrm{pH}<6.0)$.

A spacetime plot can be used to characterize the behavior of the migrating precipitation band as a function of time. ${ }^{16120}$ To construct a spacetime plot, a slice of images taken from an experiment is used. The slices are then stitched together chronologically to form a spacetime plot. An example of a spacetime plot can be seen in Figure 4.2(b). The red bar in Figure 4.2(a) indicates the slice that is used for constructing the spacetime plot in Figure 4.2(b). The slopes of the migrating precipitation band in Figure 4.2(b) give the speeds of the migrating precipitation band in the experiment. Based on Figure 4.2(b), the wave speed decreases as a function of time from $4.86 \times 10^{-4} \mathrm{~cm} \mathrm{~min}^{-1}$ to $1.81 \times 10^{-5} \mathrm{~cm} \mathrm{~min}^{-1}$.

As the migrating precipitation band moves toward the $\mathrm{AlCl}_{3} / \mathrm{HCl}$ reservoir, the 
thickness of the migrating precipitation band changes, which results in the change in the amount of transmitted light through the gel. Figure 4.3(a) shows the evolution of the average transmitted light surrounding the migrating precipitation band in terms of grayscale values over the course of the entire experiment. The migrating precipitation band starts off as a very thin precipitation band (a few microns in thickness), which permits most of the light to be transmitted resulting in high grayscale values. The migrating precipitation band thickens up to approximately 46 microns 365 minutes after the experiment has started. The migrating precipitation band starts to thin out again after it has reached the maximum thickness. Around the same time, the precipitation band also stops moving.

On top of the huge change in the average transmitted light, there are also small amplitude oscillations in the average transmitted light. This is depicted in Figure 4.3(b), which is a blow up of Figure 4.3(a) from 150 minutes to 325 minutes after the experiment has started. These small amplitude oscillations in the average transmitted light suggest that the thickness of the migrating precipitation band oscillates as the overall thickness increases. The oscillations of the average transmitted light eventually stop around 375 minutes.

A plot of the migrating precipitation band position as a function of time is superimposed onto the average transmitted light plot as shown in Figure 4.3(a). The position $0.0 \mathrm{~cm}$ is arbitrary, but it is defined so that $0.0 \mathrm{~cm}$ is closer to the $\mathrm{NaOH}$ reservoir. The migrating precipitation band starts near the $\mathrm{NaOH}$ reservoir, and it migrates toward the $\mathrm{AlCl}_{3} / \mathrm{HCl}$ reservoir. The speed of the migrating precipitation band decreases as it approaches the $\mathrm{AlCl}_{3} / \mathrm{HCl}$ reservoir. This is evident from the plot of the migrating precipitation band position as a function of time. The migrating precipitation band starts from around 150 minutes to roughly 400 minutes after the experiment has started. After 400 minutes, the position of the band stays roughly the same at around $0.5 \mathrm{~cm}$.

To study the effect of electrolyte concentrations on the migrating band speed, the 

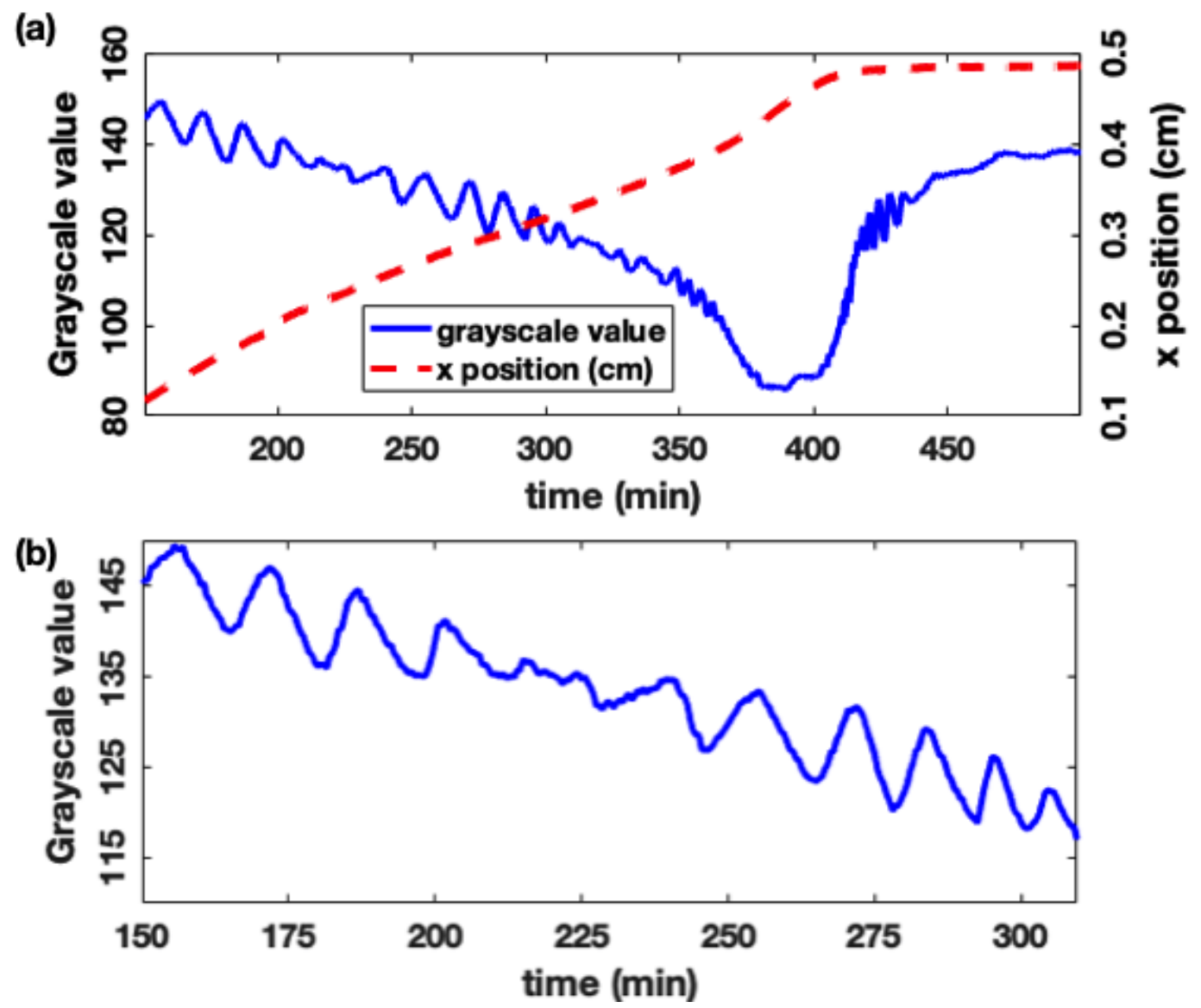

Figure 4.3: A plot of the migrating precipitation band position and its grayscale values as a function of time. The precipitation band migrates toward the $\mathrm{AlCl}_{3} / \mathrm{HCl}$ reservoir. The migration stops around 400 minutes after the band is detected. This is represented by the plateaued region around $0.5 \mathrm{~cm}$, which indicates that the band has stopped moving. While the band is migrating, the thickness of the band changes and this is shown by the oscillating grayscale values of the image. Experimental conditions are $\left[\mathrm{AlCl}_{3}\right]_{o}=0.08 \mathrm{M},[\mathrm{HCl}]_{o}=0.12$ $\mathrm{M},[\mathrm{NaOH}]_{o}=0.44 \mathrm{M}$, and $[$ agarose $]=1.0 \% \mathrm{w} / \mathrm{w}$.

appropriate chemical species concentration is varied while keeping the others constant. Figure 4.4 shows the results for the effect of electrolyte concentrations on the migrating band speed. Figure 4.4 (a) and (b) show that the migrating band speed decreases when the concentration of the aluminum chloride and hydrochloric acid are increased, respectively. On the other hand, the speed of the migrating band increases with the increase of sodium hydroxide concentration. 

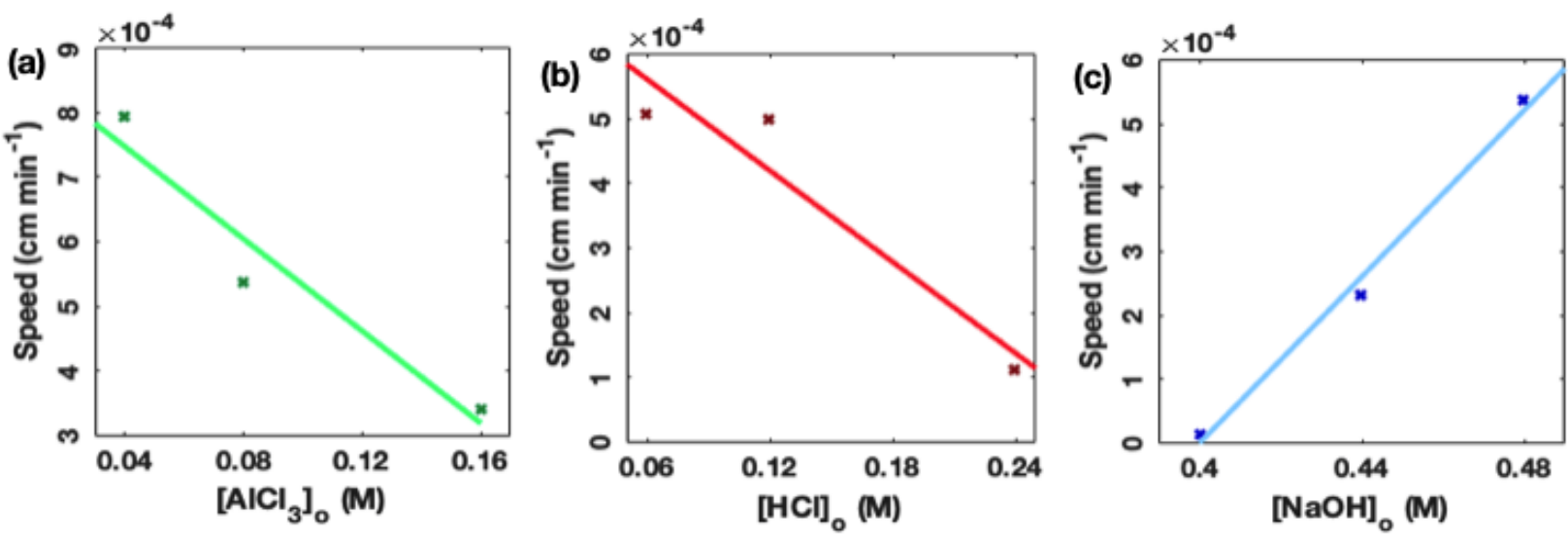

Figure 4.4: Migrating precipitation band speed as a function of (a) $\left[\mathrm{AlCl}_{3}\right]_{o}$ at $[\mathrm{HCl}]_{o}=0.12$ $\mathrm{M}$ and $[\mathrm{NaOH}]_{o}=0.48 \mathrm{M}$, (b) $[\mathrm{HCl}]_{o}$ at $\left[\mathrm{AlCl}_{3}\right]=0.08 \mathrm{M}$ and $[\mathrm{NaOH}]_{o}=0.48 \mathrm{M}$, and (c) $[\mathrm{NaOH}]_{o}$ at $[\mathrm{HCl}]_{o}=0.012 \mathrm{M}$ and $\left[\mathrm{AlCl}_{3}\right]_{o}=0.08 \mathrm{M}$. The concentration of the agarose gel is $1.0 \% \mathrm{w} / \mathrm{w}$ in all of the experiments.

\subsubsection{Front-view Experiment}

The front-view experiment allows for the observation of any patterns that formed in the gel. Typical patterns such as traveling waves and spiral patterns have been observed in this system. Figure 4.5 shows a series of images of the traveling waves observed in this system while Figure 4.6 shows the development of a spiral wave in this system. When two traveling wave fronts collide, mutual annihilation occurs as shown in Figure 4.5(b).

The same analysis can be done on the observed traveling waves using a spacetime plot. Figure 4.7(a) shows the constructed spacetime plot from snapshots of the experiment results. The spacetime plot shows that the wave speed decreases from $0.0107 \mathrm{~cm} \mathrm{~min}^{-1}$ to $0.0056 \mathrm{~cm} \mathrm{~min}^{-1}$ over the course of the experiment.

Results from the front-view experiments also show oscillations in the amount of transmitted light as evident from the changes in the grayscale value of the image. Figure 4.8 (a) shows the overall oscillations in grayscale value of a section of the image over the course of 11 hours. The large change in the grayscale values ranges from 110 at the lowest to roughly 200 at the highest. These changes occur at a larger timescale (on the order of hundreds of 

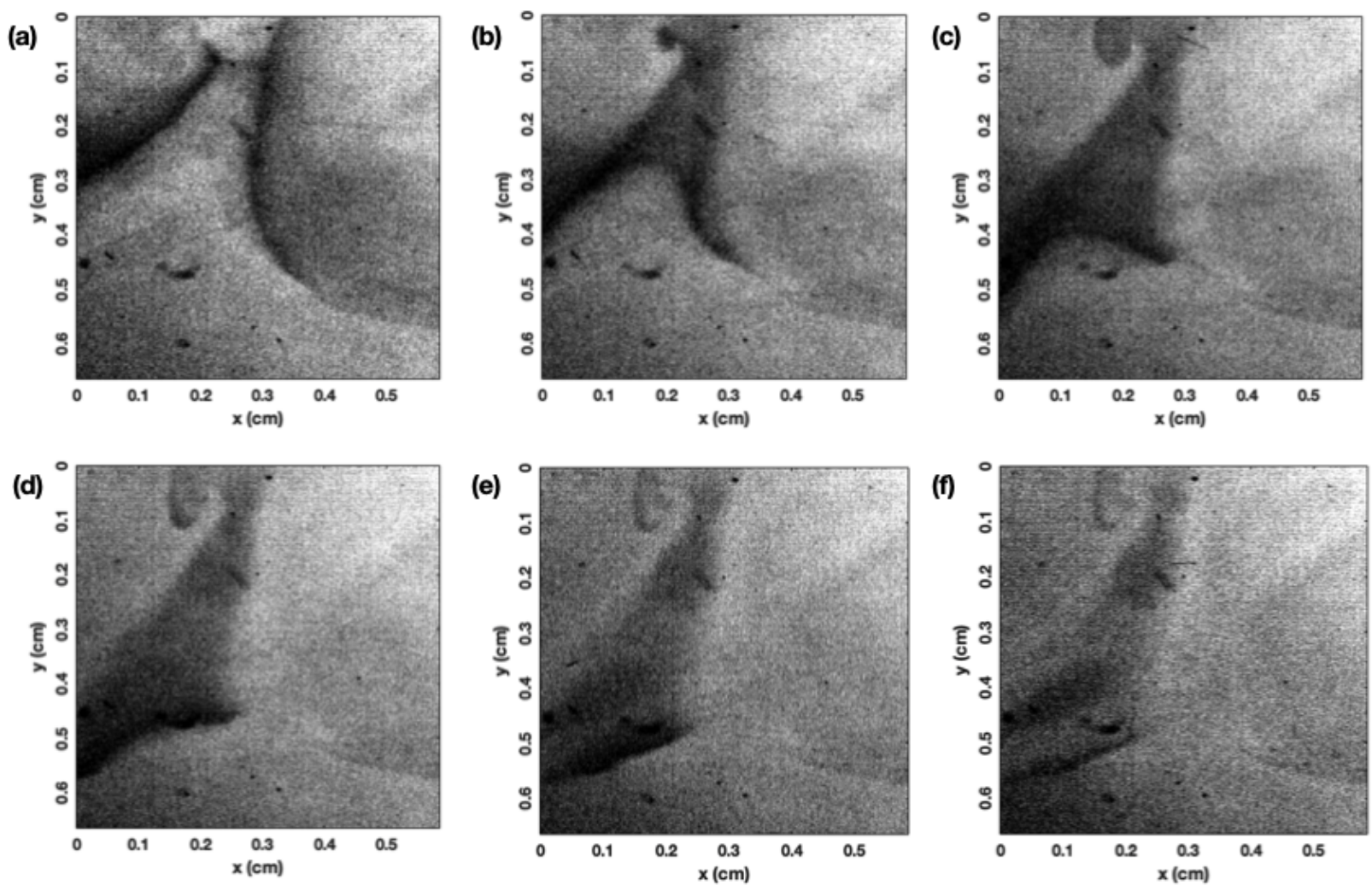

Figure 4.5: A series of pictures showing the traveling precipitating wave fronts at $\left[\mathrm{AlCl}_{3}\right]_{0}=$ $0.08 \mathrm{M},[\mathrm{HCl}]_{0}=0.12 \mathrm{M}$, and $[\mathrm{NaOH}]_{0}=0.48 \mathrm{M}$. Image (a) is taken 270 minutes after the experiment has started and the subsequent images are taken at 8 minutes apart. (b) Shows the waves annihilation as two wave fronts collide.

minute). At a smaller timescale, smaller oscillations of the grayscale values are observed. Figure $4.8(\mathrm{~b})$ shows the blow up of the small scale oscillations of the larger oscillations from the start of the experiment to roughly 200 minutes after the experiment has started. The oscillations occur in the scale of tens of minutes.

The effect of electrolyte concentrations on the traveling wave speed is also studied by varying the concentrations of the electrolyte of interest while keeping other electrolyte concentrations constant. Figure 4.9 shows the results for this part of the experiments. The traveling wave speed is observed to decrease as a function of the aluminum chloride concentration, and it increases as a function of the hydrochloric acid and the sodium hydroxide concentrations. 

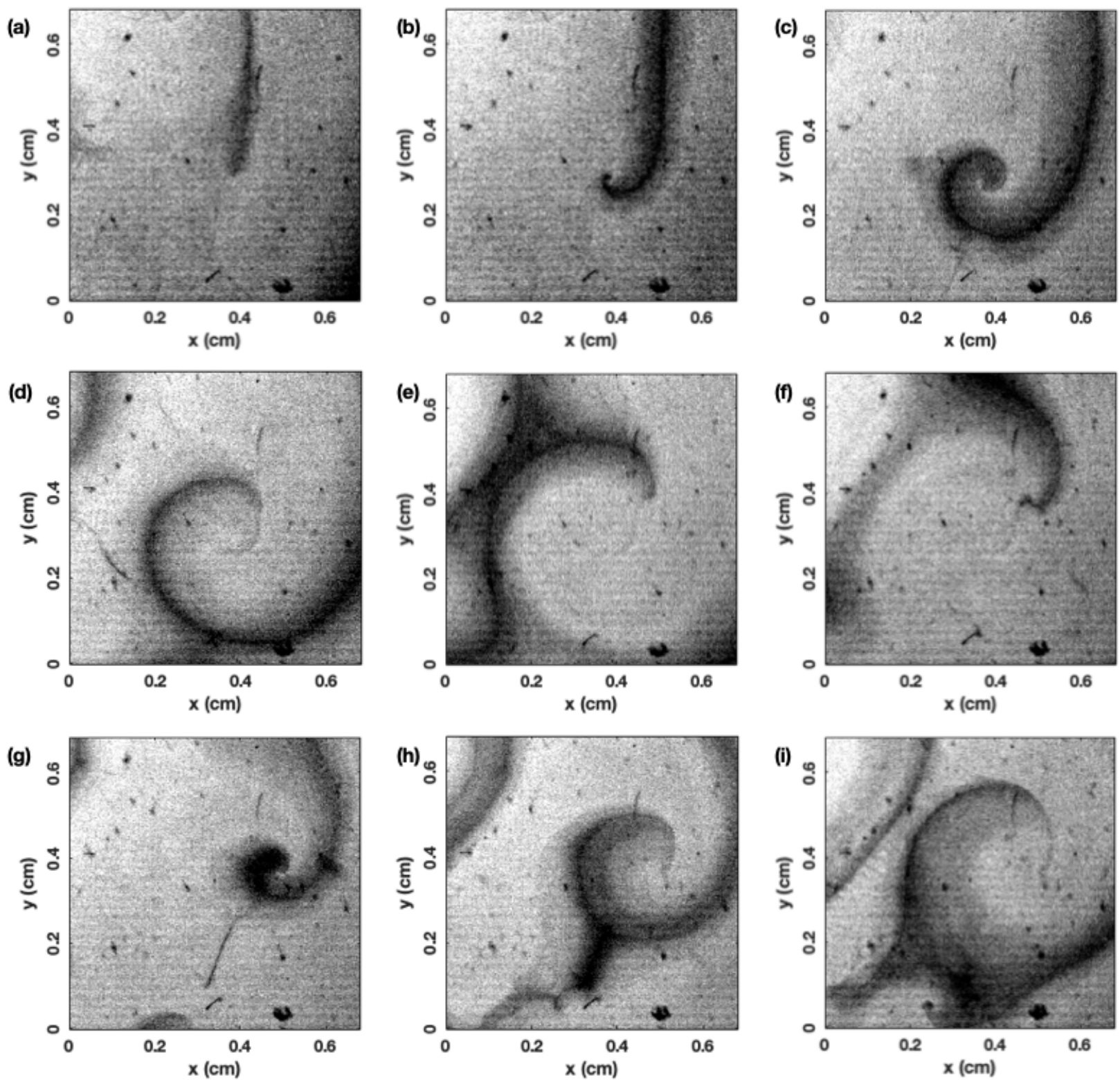

Figure 4.6: A series of pictures showing the traveling precipitation wave fronts develop into spiral waves at $\left[\mathrm{AlCl}_{3}\right]_{0}=0.075 \mathrm{M},[\mathrm{HCl}]_{0}=0.11 \mathrm{M}$, and $[\mathrm{NaOH}]_{0}=0.41 \mathrm{M}$. Image (a) is taken 170.5 minutes after the experiment has started, and the subsequent images are taken at 14 minutes apart. 

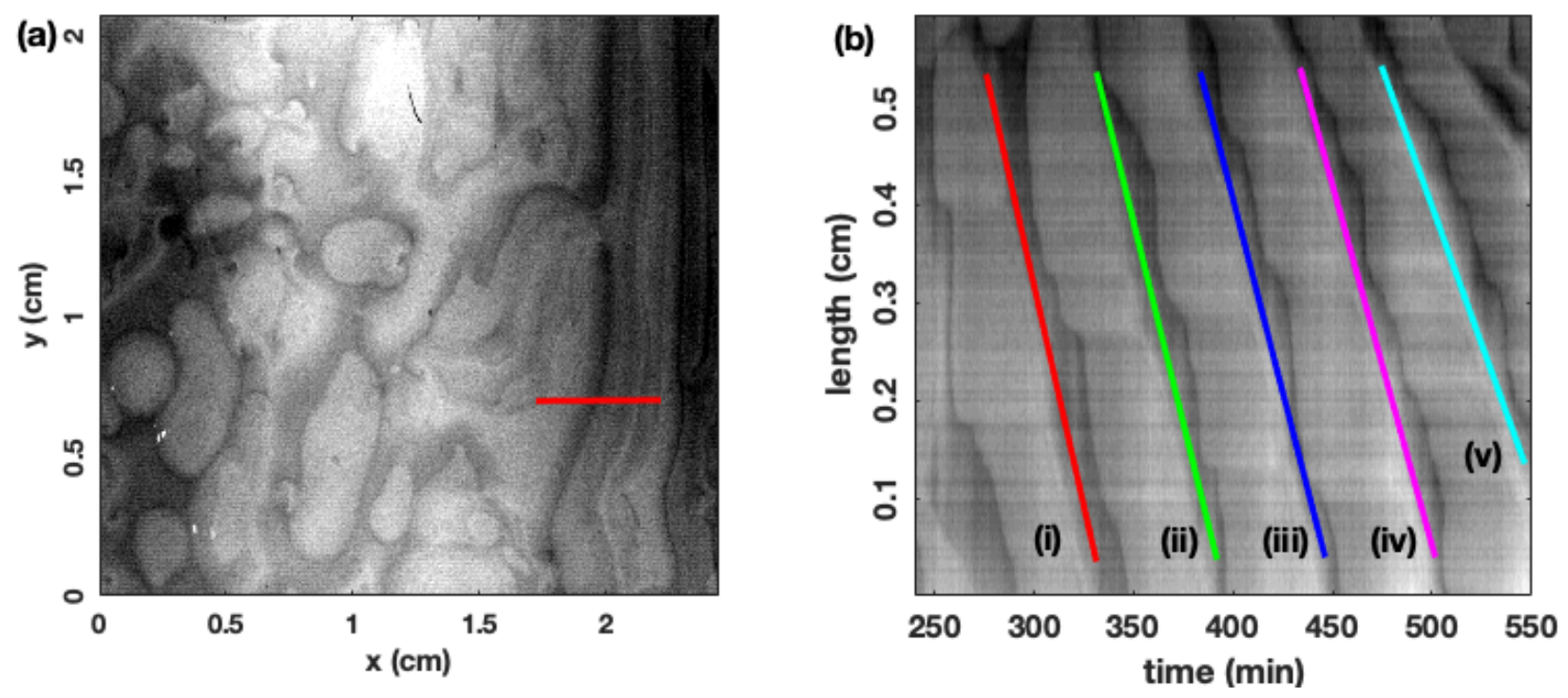

Figure 4.7: (a) A snapshot of the experiment result. The red line indicates the area used for constructing the spacetime plot. (b) A spacetime plot constructed from the experiment in (a). The slopes of the propagating waves in the spacetime plot give the speeds of the propagating waves. (i) $0.0107 \mathrm{~cm} \mathrm{~min}^{-1}$, (ii) $0.009 \mathrm{~cm} \mathrm{~min}^{-1}$, (iii) $0.0079 \mathrm{~cm} \mathrm{~min}^{-1}$, (iv) $0.0069 \mathrm{~cm} \mathrm{~min}^{-1}$, and (v) $0.0056 \mathrm{~cm} \mathrm{~min}^{-1}$. The experimental conditions are $\left[\mathrm{AlCl}_{3}\right]_{0}=0.02$ $\mathrm{M},[\mathrm{HCl}]_{0}=0.12 \mathrm{M},[\mathrm{NaOH}]_{0}=0.44 \mathrm{M}$.

\subsubsection{Simulation}

A model has been created for a closely related systems based on the Liesegang pattern phenomenon. ${ }^{1113}$ In these systems, an intermediary species is assumed to form before the formation of the aluminum hydroxide precipitate. The intermediary species called sol can diffuse within the gel, and a nucleation process of the precipitate occurs after the sol has reached a critical concentration. A model based on this idea can be created to simulate the migration of the precipitation band. In order to be consistent with the nomenclature from previous systems, aluminum chloride and sodium hydroxide will be called inner and outer electrolyte, respectively. ${ }^{412}$ The inner electrolyte, A, and the outer electrolyte, B, can react and form the diffusive intermediate, C. Species C can then form two different precipitates, $\mathrm{P}_{1}$ and $\mathrm{P}_{2}$, which are two different forms of $\mathrm{Al}(\mathrm{OH})_{3}$. In the previous systems, $\mathrm{P}_{1}$ exists in the migrating band while $\mathrm{P}_{2}$ exists in the diagonal feature of the systems. ${ }^{11 / 13}$ In these 

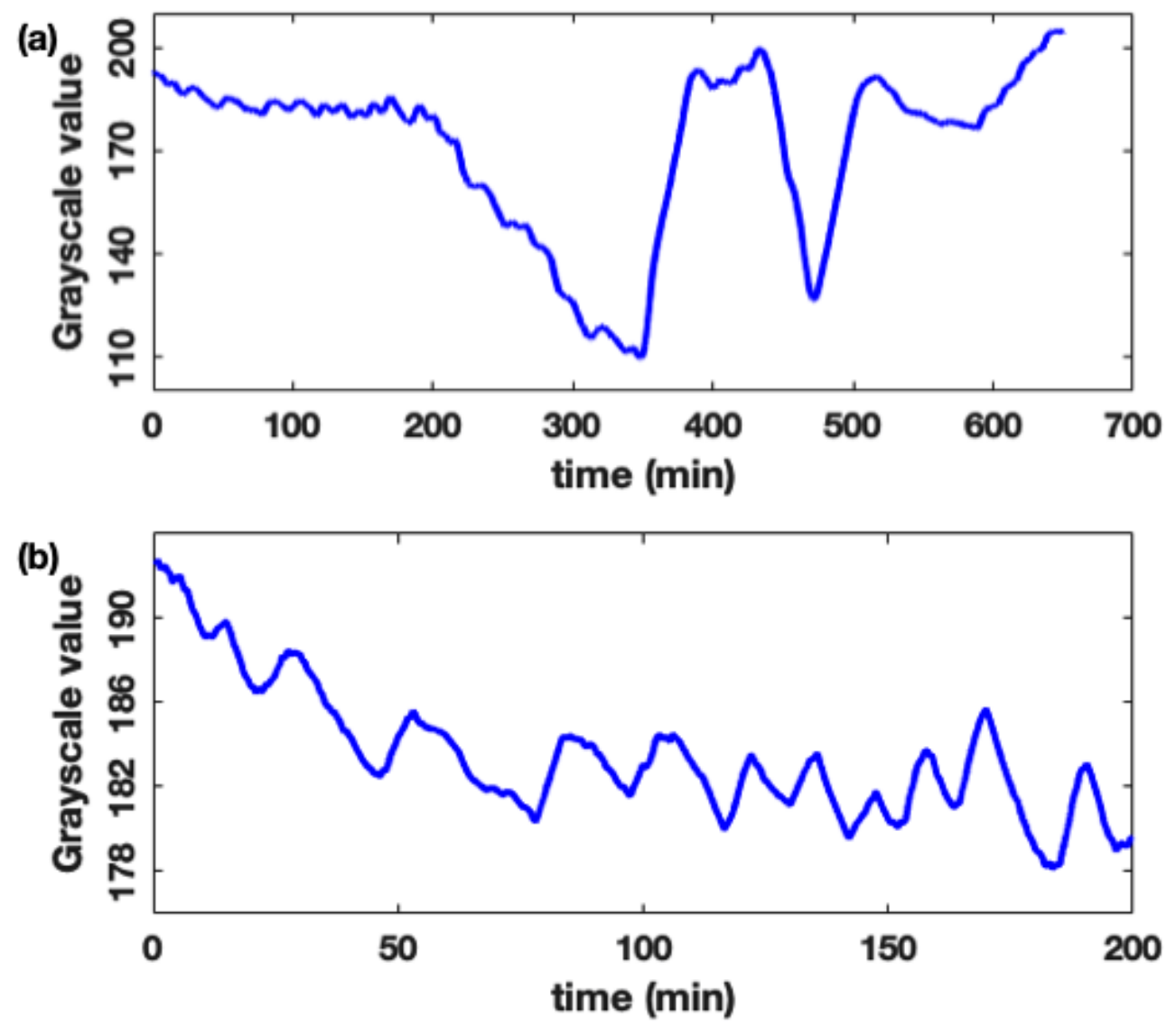

Figure 4.8: Plots showing the changes in the amount of the transmitted light in terms of grayscale values as a function of time for the front-view experiment. Higher grayscale values correspond to more transmitted light. (a) Change in grayscale value over the course of 11 hours of experiments. (b) Small amplitude oscillations that occur from 0 to $\sim 200$ minutes. The experimental conditions are $\left[\mathrm{AlCl}_{3}\right]_{0}=0.08 \mathrm{M},[\mathrm{HCl}]_{0}=0.12 \mathrm{M},[\mathrm{NaOH}]_{0}=0.44 \mathrm{M}$.
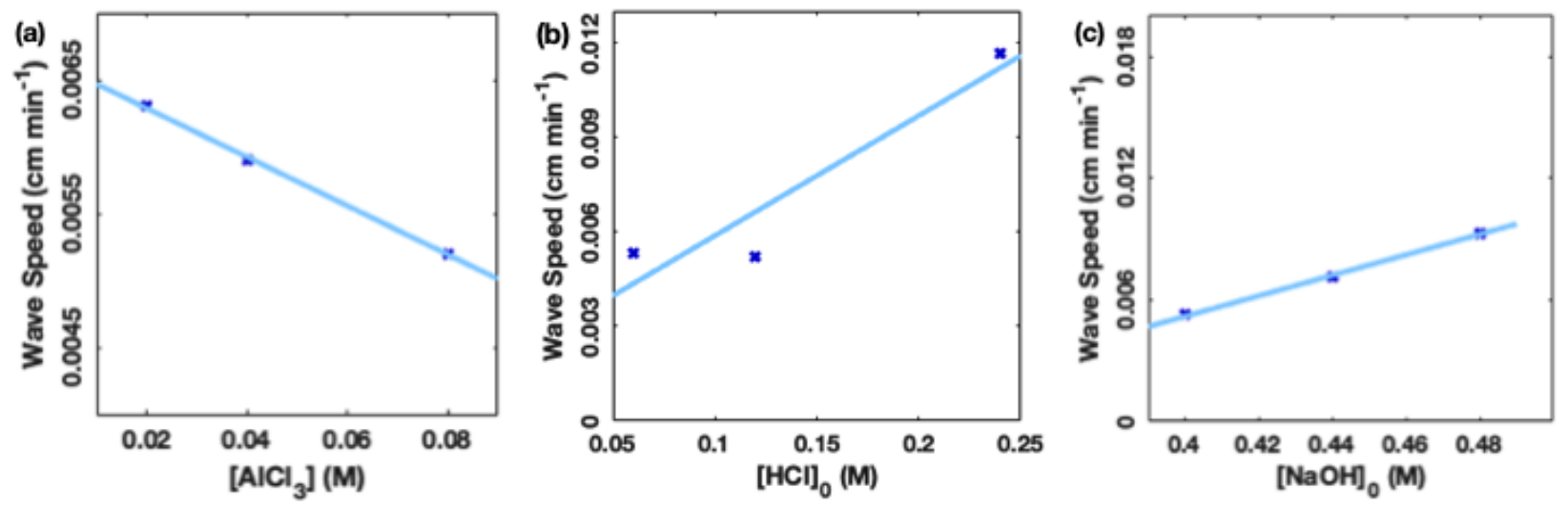

Figure 4.9: The effect of varying (a) $\left[\mathrm{AlCl}_{3}\right]_{o}$ at $[\mathrm{HCl}]_{o}=0.12 \mathrm{M}$ and $[\mathrm{NaOH}]_{o}=0.40 \mathrm{M}$, (b) $[\mathrm{HCl}]_{o}$ at $\left[\mathrm{AlCl}_{3}\right]_{o}=0.08 \mathrm{M}$ and $[\mathrm{NaOH}]_{o}=0.40 \mathrm{M}$, and $(\mathrm{c})[\mathrm{NaOH}]_{o}$ at $[\mathrm{HCl}]_{o}=0.12$ $\mathrm{M}$ and $[\mathrm{HCl}]_{o}=0.12 \mathrm{M}$ on the speed of the propagating waves. 
systems, the species $\mathrm{C}$ and $\mathrm{P}_{1}$ can further react with excess species $\mathrm{B}$ to form the inactive species. The reactions for this system can be written as follows

$$
\begin{gathered}
A+B \longrightarrow C, \\
C \longrightarrow P_{1}, \\
C+B \longrightarrow, \\
P_{1}+B \longrightarrow .
\end{gathered}
$$

The rates for the above reactions are $k_{1} a b, k_{2}\left(c-c^{*}\right) \phi\left(c-c^{*}\right), k_{3} c b$, and $k_{4} p_{1} b$, respectively. The $\phi\left(c-c^{*}\right)$ is a Heaviside step function, and it is defined as $\phi(s)=1$ for $s>1$ and $\phi(s)=0$ for $s \leqslant 0 . c^{*}$ represents the value at which the precipitation occurs.

For the system in this chapter, the addition of the hydrochloric acid adds another layer of complexity to the system. The acid, $\mathrm{H}$, can react with species $\mathrm{B}$ via a neutralization reaction. Species $\mathrm{B}$ can also react with species $\mathrm{C}$ and $\mathrm{P}_{1}$ to regenerate species $\mathrm{A}$ and species C, respectively. The reactions involving species $\mathrm{H}$ can be written as follows:

$$
\begin{gathered}
B+H \longrightarrow, \\
P_{1}+H \longrightarrow C, \\
C+H \longrightarrow A .
\end{gathered}
$$

The rates for these additional reactions are $k_{5} b h, k_{6} p_{1} h$, and $k_{7} c h$. The reaction- 
diffusion equation of this system can be written as follows:

$$
\begin{aligned}
\frac{\partial a}{\partial t} & =D_{a} \nabla^{2} a-k_{1} a b+k_{6} c h, \\
\frac{\partial b}{\partial t} & =D_{b} \nabla^{2} b-k_{1} a b-k_{3} c b-k_{1} p_{1} b-k_{7} b h, \\
\frac{\partial c}{\partial t} & =D_{c} \nabla^{2} c+k_{1} a b-k_{2}\left(c-c^{*}\right) \phi\left(c-c^{*}\right)-k_{3} c b+k_{6} c h, \\
\frac{\partial p_{1}}{\partial t} & =k_{2}\left(c-c^{*}\right) \phi\left(c-c^{*}\right)-k_{4} p_{1} b-k_{5} p_{1} h, \\
\frac{\partial h}{\partial t} & =D_{h} \nabla^{2} h-k_{5} p_{1} h-k_{6} c h-k_{7} b h,
\end{aligned}
$$

where a, b, c, $p_{1}$, and h are the concentration of inner electrolyte, outer electrolyte, diffusive intermediate, precipitate type 1 , and acid. $D_{a}, D_{b}, D_{c}, D_{h}$ are the diffusion coefficient of the inner electrolyte, the outer electrolyte, the diffusive intermediate species, and the acid, respectively, They are set to $5 \times 10^{-4}, 1 \times 10^{-3}, 2 \times 10^{-4}$, and $2 \times 10^{-3}$, respectively. The rate constants $\mathrm{k}_{1}, \mathrm{k}_{2}, \mathrm{k}_{3}, \mathrm{k}_{4}, \mathrm{k}_{5}, \mathrm{k}_{6}$, and $k_{7}$ are set to $3.0 \times 10^{2}, 8.0 \times 10^{-3}, 7.5 \times 10^{1}, 9.5 \times 10^{-1}$, $2 \times 10^{4}, 8 \times 10^{-1}$, and $3 \times 10^{4}$, respectively.

The model is created for a one-dimensional system, and the reaction-diffusion equations are solved using Euler's method with grid spacing, $\Delta x$, of 0.01 and timestep, $\Delta t$, of $5.5 \times 10^{-4}$. The boundary condition is set to no-flux for all species except for $a(t, L)=a_{0}$, $b(t, 0)=b_{o}$, and $h(t, L)=h_{o}, \forall \mathrm{t}$. The initial condition is set to be $c(0, x)=0, p_{1}(0, x)=0$, $\forall \mathrm{x} ; a(0, L)=a_{0}, b(0,0)=b_{o}$, and $h(0, L)=h_{o}$ where $\mathrm{L}$ is the length of the gel, and it is set to 100 .

Figure 4.10 shows the simulation results for the migrating precipitation band based on the the reaction-diffusion reaction in equations 4.8 to 4.12 . Figure 4.10(a) shows the spacetime plot of the gel $\mathrm{pH}$ while Figure 4.10(b) shows the spacetime plot of the precipitate concentration. By comparing these two spacetime plots, it is evident that the migrating band exists in a $\mathrm{pH}$ range of $\sim 4$ to 9 . When the medium $\mathrm{pH}$ is less than $\sim 4$, the aluminum species exists as aluminum ions whereas when the medium $\mathrm{pH}$ is greater than 9 , the aluminum 

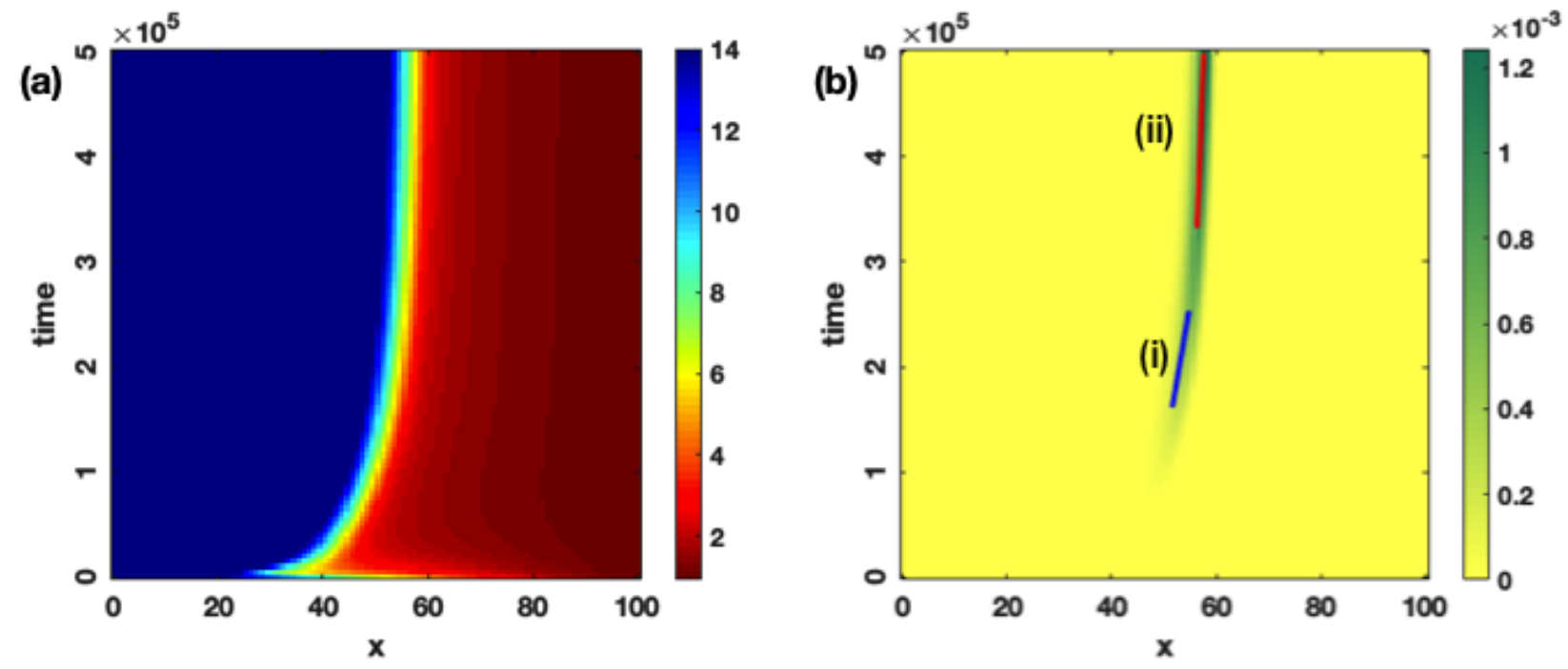

Figure 4.10: Results from simulation for the migrating band in this system. (a) The spacetime plot for the $\mathrm{pH}$. (b) The spacetime plot from the simulation for the concentration of $\mathrm{AlCl}_{3}$ precipitate.

species exists mostly in the form of aluminum hydroxide complex ion, $\mathrm{Al}(\mathrm{OH})_{4}^{-}$.

The slope of the bars in Figure 4.10(b) can be used to determine the dimensionless speed of the migrating precipitation band. Slope (i) has a value of $3.44 \times 10^{-5}$ while slope (ii) has a value of $7.62 \times 10^{-6}$. Slope (i) is an order of magnitude greater than the slope (ii), which is similar to what is observed in the experiment. The effect of the electrolytes concentration on the simulated migrating precipitation band can be studied by changing the initial concentration of one electrolyte while keeping the other two electrolyte concentrations constant.

\subsection{Discussion}

In previous systems, the migrating precipitation band typically has its thickness monotonously increasing as the experiment progresses. The migrating band thickness keeps increasing until it has reached its maximum thickness of approximately $200 \mu \mathrm{m}$. The migrating band speed typically ranges from $1 \times 10^{-3} \mathrm{~cm} \mathrm{~min}^{-1}$ to $1 \times 10^{-2} \mathrm{~cm} \mathrm{~min}^{-1}$. Both the 

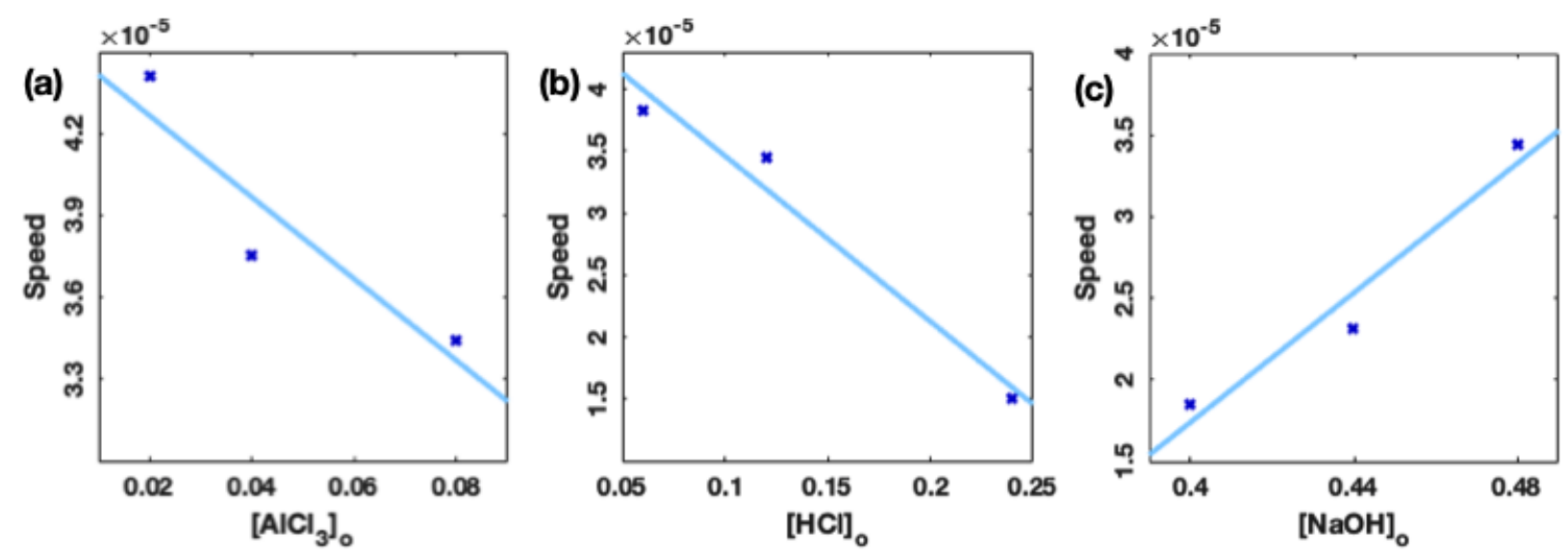

Figure 4.11: The effect of electrolytes concentration on the simulated migrating precipitation band. The conditions for the simulations are (a) (a) vary $\left[\mathrm{AlCl}_{3}\right]_{o}$ at $[\mathrm{HCl}]_{o}=0.12 \mathrm{M}$ and $[\mathrm{NaOH}]_{o}=0.48 \mathrm{M}$, (b) vary $[\mathrm{HCl}]_{o}$ at $\left[\mathrm{AlCl}_{3}\right]_{o}=0.08 \mathrm{M}$ and $[\mathrm{NaOH}]_{o}=0.48 \mathrm{M}$, and (c) vary $[\mathrm{NaOH}]_{o}$ at $[\mathrm{HCl}]_{o}=0.12 \mathrm{M}$ and $\left[\mathrm{AlCl}_{3}\right]_{o}=0.08 \mathrm{M}$ on the speed of the migrating precipitation band.

migrating band thickness and its speed are dependent on the concentration of the electrolytes used. $\underline{41112}$

In contrast to the previous systems, the thickness of the migrating precipitation band in the system of this chapter increases as the experiment progresses with small amplitude oscillations. These small amplitude oscillations are captured in Figure 4.3 . The thickness of the migrating band continues to increase with small oscillations until the maximum thickness of approximately $50 \mu \mathrm{m}$ has been reached. Then, the migrating precipitation band starts to undergo a thinning process, which can be attributed to the dissolution process from both sides of the band. The migrating precipitation band speed in this system ranges from $1 \times 10^{-5} \mathrm{~cm} \mathrm{~min}^{-1}$ to $1 \times 10^{-6} \mathrm{~cm} \mathrm{~min}^{-1}$, which makes the migrating band speed of this system at least one magnitude smaller than its predecessors.

The difference in the behaviors of these two systems, the system of this chapter and the system from previous studies, can be attributed to the addition of hydrochloric acid into the system. The addition of the hydrochloric acid into the system creates a steep $\mathrm{pH}$ gradient at the regions where the $\mathrm{H}^{+}$and $\mathrm{OH}^{-}$ions meet as shown in Figure 4.10. This region of steep 
pH gradient migrates toward $A l^{3+} / H^{+}$reservoir and eventually it remains stationary. Since $\mathrm{Al}(\mathrm{OH})_{3}$ precipitate can only exist within a $\mathrm{pH}$ range of $\sim 4$ to $9.5, \mathrm{Al}(\mathrm{OH})_{3}$ precipitate is expected to exist only within the region with the steep pH gradient. Figure 4.10 shows that this is the case.

The effect of the electrolyte concentrations on the migrating precipitation band speed is also captured by the simulation since the simulation results agree with the experimental observations. An increase in the sodium hydroxide concentration results in an increase in the migrating precipitation band speed. On the other hand, an increase in the concentration of aluminum chloride or hydrochloric acid results in a decrease in the migrating precipitation band speed. These observations can be understood in term of each electrolyte concentration profile.

In the case of the hydroxide ions, the concentration of the hydroxide ions is high near its reservoir and low near the $\mathrm{AlCl}_{3} / \mathrm{HCl}$ reservoir. This concentration profile results in the net diffusion of hydroxide ions toward $\mathrm{AlCl}_{3} / \mathrm{HCl}$ reservoir. From the experimental observations, the precipitation band migrates toward the $\mathrm{AlCl}_{3} / \mathrm{HCl}$ reservoir. Thus, the hydroxide ions can be viewed as a "pushing" force that accelerates the migration of the precipitation band toward the $\mathrm{AlCl}_{3} / \mathrm{HCl}$ reservoir.

In the case of aluminum ions and hydrogen ions, the concentration of these ions are high near the $\mathrm{AlCl}_{3} / \mathrm{HCl}$ reservoir and low near the $\mathrm{NaOH}$ reservoir. This concentration profile results in a net diffusion of the aluminum and hydrogen ions toward the $\mathrm{NaOH}$ reservoir. This net diffusion of aluminum ions and hydrogen ions toward $\mathrm{NaOH}$ is the opposite direction of the migrating precipitation band movement. Thus, the aluminum ions and hydrogen ions can be viewed as a "retarding" force that slows down the migration of the precipitation band. Once the "pushing" and the "retarding" forces are equal, the migrating precipitation band stops moving as shown in Figure 4.3(a). 
The thickness oscillations of the migrating precipitation band can also be observed from the front-view experiment since the chemical reactor is lit from the back. Regions with a thinner migrating precipitation band allow more light to be transmitted while regions with a thicker migrating precipitation band allow less light to be transmitted. This behavior is captured in Figure 4.8, which shows the oscillations of the transmitted light in terms of the grayscale values from the front-view experiments. Ideally, the thickness oscillations observed from the side-view and the front-view should be closely matched in time. However, that is not the case since the thickness oscillations started immediately for front-view experiment whereas for the side-view experiment, the oscillations only start roughly around 150 minutes after the experiment has started. The difference in timing can be attributed to the different thickness of the agarose gel used. The thickness of the agarose gel used in the front-view experiment is approximately $5 \mathrm{~mm}$. The thickness of the gel in the front-view experiment is equivalent to the width of the agarose gel in the side-view experiment. This is because the electrolytes in the front-view experiment diffused into the agarose gel from the front and the back, whereas in the side-view experiment, the electrolytes diffused from the sides of the agarose gel. The width of the agarose gel in the side-view experiment is $10 \mathrm{~mm}$, which is twice the thickness of the agarose gel in the front-view experiment. Since the "thickness" is two times greater in the side-view experiment, it takes a longer time for the migrating precipitation band to form in this setup compared to the front-view experimental setup. Thus, the thickness oscillations are only observed at later time and not immediately.

\subsection{Conclusion}

The system in this chapter can be considered as an extension of previously studied systems. The addition of the hydrochloric acid into the system allows for the creation 
and maintenance of a $\mathrm{pH}$ gradient across a thin region where the sodium hydroxide and the hydrochloric acid meet. This steep $\mathrm{pH}$ gradient migrates toward the aluminum chloride/hydrochloric acid reservoir. The thin region of the steep $\mathrm{pH}$ gradient allows for the existence of aluminum hydroxide precipitate since the $\mathrm{pH}$ in this region varies from approximately 4 to 10 . The oscillatory behavior of the propagating precipitation band is observed from the side-view and the front-view experiments. However, the oscillations cannot be matched temporally due to the limitation imposed by the reactors used. 


\subsection{References}

[1] S. Camazine, J.-L. Deneubourg, N. R. Franks, J. Sneyd, G. Theraulaz, and E. Bonabeau, in Self-Organization in Biological Systems (Princeton University Press, 2001) pp. $7-13$.

[2] A. N. Zaikin and A. M. Zhabotinsky, Nature 225, 535 (1970).

[3] K. H. Stern, Chem. Rev. 54, 79 (1954).

[4] A. Volford, F. Izsák, M. Ripszám, and I. Lagzi, Langmuir 23, 961 (2007).

[5] J. P. Keener and J. J. Tyson, Phys. D 21, 307 (1986).

[6] Y.-F. Liu, Y.-N. Wu, H.-J. Xu, and J.-F. Sun, Communications in Theoretical Physics 42, $637(2004)$.

[7] A. Winfree and W. Jahnke, J. Phys. Chem. 93, 2823 (1989).

[8] T. Bánsági and O. Steinbock, Chaos 18, 026102 (2008).

[9] A. Toramaru and T. Harada and T. Okamaru, Phys. D 183, 133 (2003).

[10] H. Batlouni and M. Al-Ghoul, J. Phys. Chem. A 112, 8038 (2008).

[11] M. R. Tinsley, D. Collison, and K. Showalter, J. Phys. Chem. A 117, 12719 (2013).

[12] M. M. Ayass, M. Al-Ghoul, and I. Lagzi, J. Phys. Chem. A 118, 11678 (2014).

[13] M. Tinsley, D. Collison, and K. Showalter, Chaos 25, 064306 (2015).

[14] M. M. Ayass, I. Lagzi, and M. Al-Ghoul, Phys. Chem. Chem. Phys. 16, 24656 (2014).

[15] M. M. Ayass, I. Lagzi, and M. Al-Ghoul, Phys. Chem. Chem. Phys. 30, 19806 (2015). 
[16] B. Dúzs, I. Lagzi, and I. Szalai, Langmuir 30, 5460 (2014).

[17] F. Molnár, L. Roszol, A. Volford, and I. Lagzi, Chem. Phys. Lett. 503, 231 (2011).

[18] M. M. Emamjomeh, M. Sivakumar, and A. S. Varyani, Desalination 275, 102 (2011).

[19] K. Kurin-Csörgei, I. R. Epstein, and M. Orbán, Letters to Nature 433, 139 (2005).

[20] N. Manz, C. T. Hamik, and O. Steinbock, Phys. Rev. Lett. 92, 248301 (2004). 


\section{Chapter 5}

\section{Turbulence-like Patterns in the}

\section{Aluminum Hydroxide System}

\section{$5.1 \quad$ Introduction}

Self-organized patterns have been observed and studied in many fields including chemistry, cardiology, and biology. ${ }^{113}$ Studies on spiral waves in the heart lead to the understanding of the cardiac diseases such as ventricular fibrillation and ventricular tachycardia.2 In carbon monoxide conversion catalysis, the understanding of pattern formations on the platinum surface helps engineers to create a better catalyst for carbon monoxide to carbon dioxide conversion. ${ }^{4}$

In chemistry, self-organized patterns have been extensively studied using the BelousovZhabotinsky (BZ) reaction or some variations of it. Studies on the BZ reaction mechanism showed that the BZ reaction consists of several intermediate species and several elementary reactions. ${ }^{516}$ If the $\mathrm{BZ}$ solution is left unstirred, the solution is known to produce patterns such as spiral patterns and target patterns in a two-dimensional system. ${ }^{[78}$ In a three-dimensional system, an unstirred BZ solution can produce scroll waves. ${ }^{9}$ Scientists observed that spiral 
patterns and target patterns can transition to turbulent patterns via several mechanisms.

For example, in the BZ system, the spiral waves tips have been shown to meander when the malonic acid concentration was lowered beyond the critical concentration. The meandering tips cause spiral wave patterns to transition to turbulent patterns via the Doppler instability. $10[11$ If the malonic acid concentration and the sulfuric acid concentration are varied simultaneously past their critical points, the spiral patterns would transition to the turbulent patterns via the long wavelength instability. 11

Target patterns and spiral patterns have also been reported in precipitation systems. These patterns have been documented to occur in the aluminum chloride/sodium hydroxide system and the mercuric chloride/potassium iodide system. 12 Although spiral patterns and target patterns have been reported in these systems, the underlying mechanisms for the pattern formation are different. In the case of the BZ reaction, the autocatalytic reaction coupled with the diffusion promote the formation of these patterns. ${ }^{7}$ On the other hand, the precipitation and the redissolution reactions together with the diffusion of the chemical species are responsible for the patterns formed in the precipitation systems. ${ }^{15}$ In the case of the aluminum chloride/sodium hydroxide system the following chemical reactions take place:

$$
\begin{gathered}
\mathrm{Al}_{(a q)}^{3+}+3 \mathrm{OH}_{(a q)}^{-} \rightarrow \mathrm{Al}(\mathrm{OH})_{3}{ }_{(s)}, \\
\mathrm{Al}(\mathrm{OH})_{3(s)}+\mathrm{OH}_{(a q)}^{-} \rightarrow\left[\mathrm{Al}(\mathrm{OH})_{4}\right]_{(a q)}^{-} .
\end{gathered}
$$

Equation 5.1 is the precipitation reaction while equation 5.2 is the complex forming redissolution reaction.

The spiral patterns and the target patterns formed have been shown to be robust across several precipitation systems. With the increase of the inner electrolyte concentrations, the number of the nucleation centers and the pattern wavenumbers have been observed to increase. ${ }^{12115}$ The precipitation systems also show that the number of the nucleation cen- 
ters increases as a function of time, and this same behavior has been reported in the BZ systems. $\frac{10 \mid 12}{12}$

In this chapter, the existence of turbulence-like patterns in the propagating precipitation system of $\mathrm{AlCl}_{3} / \mathrm{NaOH}$ is studied. Target patterns and spiral patterns can transition to turbulence-like patterns as the concentrations of the aluminum chloride are increased. The resulting patterns formed in this system are characterized using the spatial correlation function, the source or sink identification technique, the temporal standard deviations of the velocity angles, the spatial means of the velocity angle temporal standard deviations, and the spatial standard deviation of the velocity angle temporal standard deviations.

\subsection{Method}

Figure 5.1 shows the experimental setup used in this study. The agarose gel is prepared by mixing the appropriate amount of agarose powder in water to make a $1 \%$ agarose/water mixture. The mixture is then heated and stirred continuously until the temperature of the mixture reaches $90^{\circ} \mathrm{C}$, and all of the agarose powder is completely dissolved. The hot agarose gel is stirred for an additional 5 minutes with the heat turned off before aluminum chloride hexahydrate salt is added into the hot agarose gel. The amount of aluminum chloride hexahydrate salt added depends on the initial concentration of aluminum chloride for a particular experiment. The hot agarose gel is then transferred into a petri dish to make a gel with $\sim 1 \mathrm{~cm}$ thickness, and the gel is left to cool down and solidify at room temperature. Once the agarose gel has solidified, $2.5 \mathrm{M}$ sodium hydroxide is poured onto the gel. The petri dish is illuminated from the bottom for all of the experiments. The image of the patterns formed are taken every $30 \mathrm{~s}$ using a camera that is attached to a computer with MATLAB installed. 

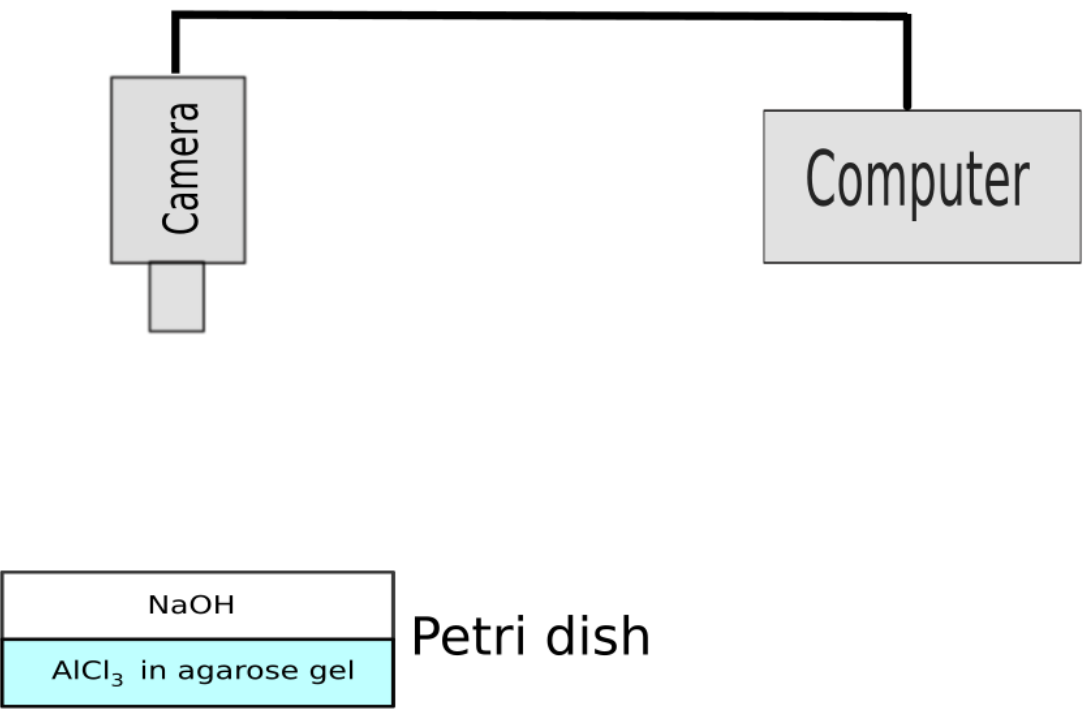

\section{Light source}

Figure 5.1: The experimental setup used to record the patterns formed in the $1 \%$ agarose gel. The recorded pattern images from this setup are used for the analysis to characterize the behavior of the waves that made up the patterns.

\subsection{Results}

\subsubsection{Image Acquisition and Velocity Determination}

Target patterns and spiral patterns generally formed at low initial concentrations of the aluminum chloride $(\leqslant 0.31 M)$. Figure 5.2 (a) shows a target pattern that forms in the agarose gel with $0.30 M$ of the aluminum chloride. As the initial concentrations of the aluminum chloride in the agarose gel are increased, more initiation centers appear which result in the formation of the turbulence-like patterns. The turbulence-like patterns start to appear when $0.35 \mathrm{M}$ of the initial concentration of the aluminum chloride is used. The turbulence-like patterns appear to be more crowded as the initial concentration of the 
aluminum chloride is increased. This can be seen in Figure 5.2 .

A technique called optical flow, which is readily available in programming software such as MATLAB, is used to characterize the patterns. This technique allows us to determine the velocity of objects in images by comparing images taken at different times. For target patterns and spiral patterns such as in Figure 5.2(a) and (b), respectively, the velocity field has an outward direction originating from the center of the patterns. This same behavior is also observed at $0.35 \mathrm{M}$ of the aluminum chloride albeit there are multiple centers that appear and no intact target patterns are observed. At higher initial concentrations of the aluminum chloride, no discernible center can be detected from the velocity field of the propagating waves. The outward flow of the propagating waves from the center makes the center a source. The existence of a source suggests that there might be a center that acts like a sink. Therefore, some techniques can be used to determine the source or sink in the image based on the velocity fields of the propagating waves.

\subsubsection{Source or Sink Identification Technique}

One of the simplest ways to determine the source or sink of a system based on its vector field is to take the divergence of the vector field for that system. $\frac{16}{\text { The divergence of }}$ the vector field can be calculated as follows:

$$
\nabla \cdot \mathbf{V}=\frac{\partial u}{\partial x}+\frac{\partial v}{\partial y}
$$

A positive divergence value indicates that the region is a source whereas a negative divergence indicates that the region is a sink. Figure 5.3 shows the colormap of the divergence of the velocity fields for the propagating precipitation waves at various concentrations. The velocity

fields of the propagating precipitation waves are superimposed on top of the colormaps at various concentrations as shown in Figure 5.3 . 

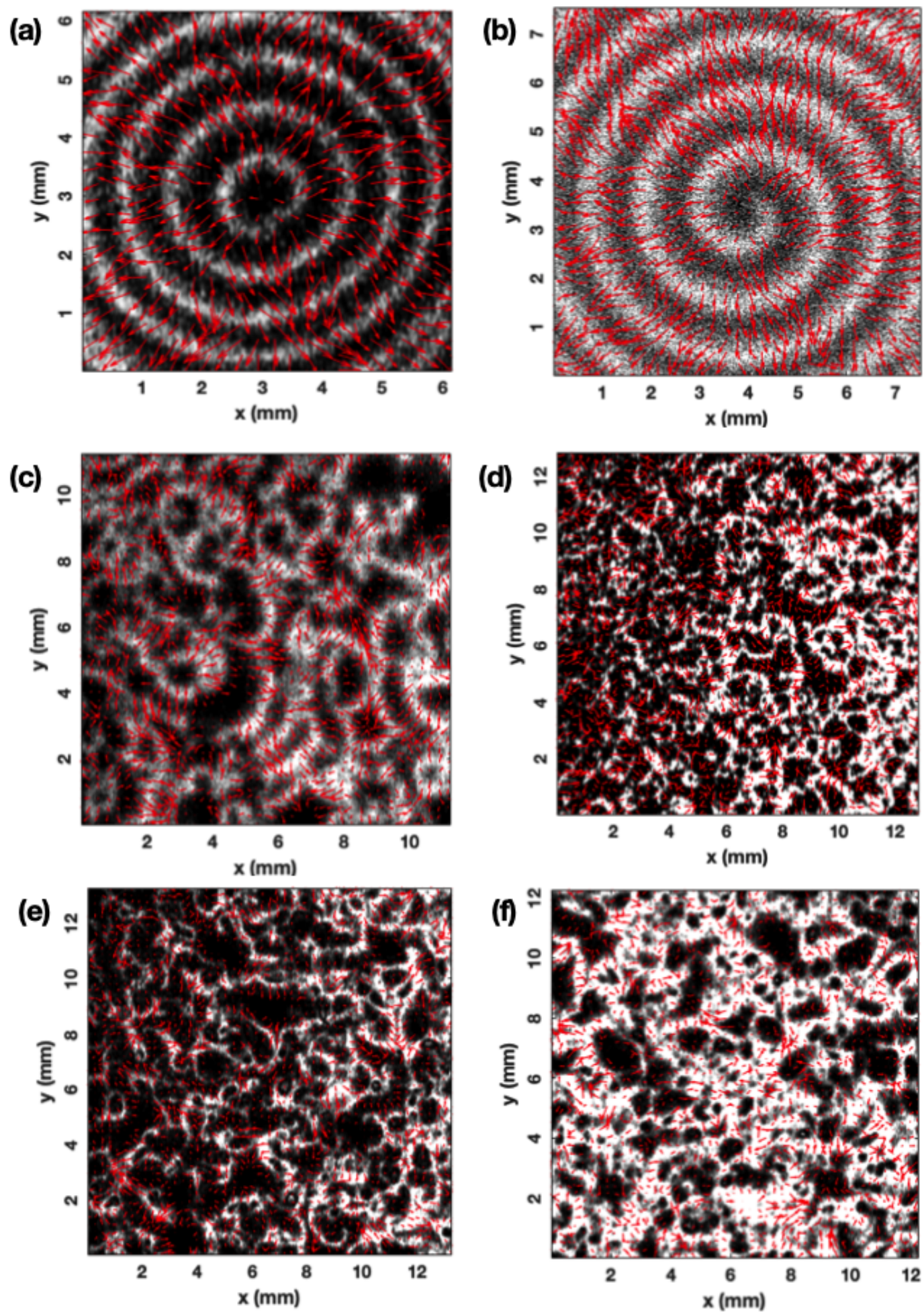

Figure 5.2: A series of images of the traveling waves that form in the agarose gel at various initial concentration of the aluminum chloride. $\left[\mathrm{AlCl}_{3}\right]_{0}$ : (a) $0.30 \mathrm{M}$, (b) $0.31 \mathrm{M}$, (c) $0.35 \mathrm{M}$, (d) $0.40 \mathrm{M}$, (e) $0.43 \mathrm{M}$, and (f) $0.45 \mathrm{M}$. 

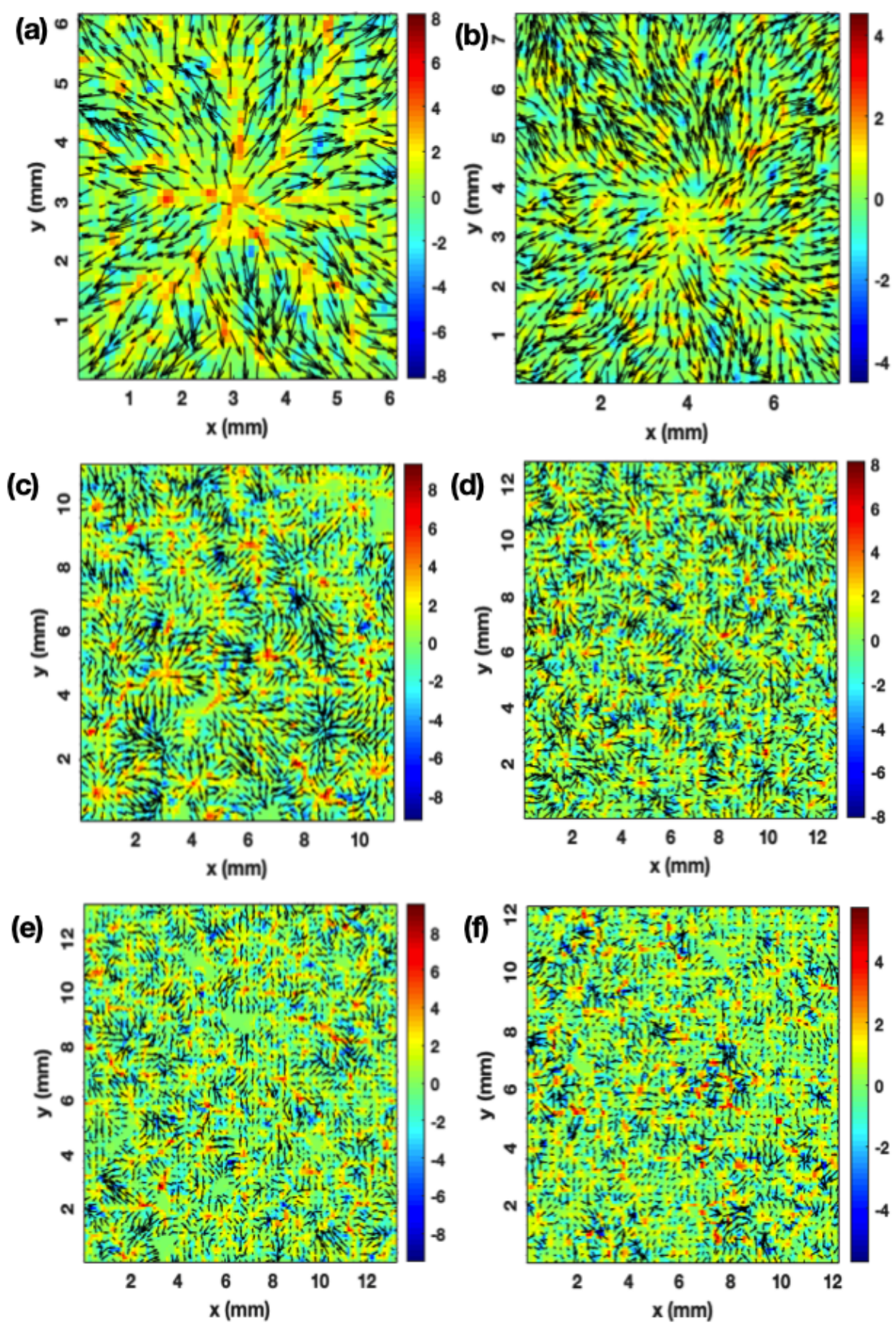

Figure 5.3: Velocity fields of the propagating precipitation waves at different initial concentrations of the aluminum chloride. $\left[\mathrm{AlCl}_{3}\right]_{0}$ : (a) $0.30 \mathrm{M}$, (b) $0.31 \mathrm{M}$, (c) $0.35 \mathrm{M}$, (d) 0.40 $\mathrm{M}$, (e) $0.43 \mathrm{M}$, and (f) $0.45 \mathrm{M}$. The color represents the strength of the source or sink at different locations within the gel. 


\subsubsection{Spatial Correlation Function}

To characterize the patterns formed in these gels, a two-point piecewise spatial correlation function is used. This spatial correlation function was used in the past to characterize patterns formed in the BZ system. ${ }^{1}$ The spatial correlation function can be written as follows:

$$
\begin{aligned}
A(j, t) & =\frac{1}{\sigma_{N}^{2}} \sum_{i=1}^{N} \delta I_{i, t} \delta I_{i+j, t}, \\
\delta I_{i+j, t} & =I_{i+j, t}-I_{t} \\
\delta I_{i, t} & =I_{i, t}-I_{t} \\
I_{t} & =\frac{1}{N} \sum_{i=i}^{N} I_{i, t}
\end{aligned}
$$

where $I_{i, t}$ is the intensity (grayscale values) at pixel $i$ and time $t$, and $I_{t}$ is the average intensity of the whole image at time $t . \delta I_{i, t}$ is the difference between the intensity at pixel $i$ and the average intensity of the entire image at time $t$. To calculate the spatial correlation values for a two-dimensional image, the intensity at pixel $i$ is compared to the intensity at pixel $j$ away. Since there are multiple pixels $j$ away from the pixel $i$, pixels $j$ resemble a ring surrounding pixel $i$. To smoothen the ring in the discrete array, the intensity at pixel $i$ is compared to the average intensity of pixels within $j \pm 1$ from pixel $i$, and this average is assigned to $I_{i+j, t} . \delta I_{i+j, t}$ is the difference between $I_{i+j, t}$ and $I_{t} . \sigma_{N}^{2}$ is the variance of the image intensity.

Figure 5.4 shows the effect of different initial concentrations of the aluminum chloride on the resulting spatial correlation function plots. At low initial concentrations of the aluminum chloride $(\leqslant 0.31 \mathrm{M})$, the spatial correlation function plots have oscillating spatial correlation values as can be seen in Figure 5.4(a) and (b). At these concentrations, target patterns and spiral patterns are observed in the agarose gel as shown in Figure 5.2(a) and (b), respectively. At higher concentrations of the aluminum chloride $(\geqslant 0.35 \mathrm{M})$, these 
oscillating values decay to zero as shown in Figure 5.4 (c)-(f).

\subsubsection{Temporal Standard Deviation of the Velocity Angles}

The analyses performed so far focus primarily on the behavior in the spatial domain. To characterize the behavior of the system in the time domain, the temporal standard deviation of the propagating wave velocity angles can be calculated. The results for such calculation are shown in Figure 5.5. For target patterns and the spiral patterns, the temporal standard deviation of the propagating wave velocity angles has low standard deviations except at the center of the target patterns and the spiral patterns. The center of the target patterns and spiral patterns have high temporal standard deviations. On the other hand, the turbulence-like patterns have high temporal standard deviations throughout the reaction medium.

This analysis can be taken a step further by calculating the spatial means and the spatial standard deviations for the temporal standard deviations of the velocity angles. Figure 5.6 shows the plot of the spatial means of the temporal standard deviations as a function of the initial concentrations of the aluminum chloride. The bar on each point represents the spatial standard deviation of the temporal standard deviation at a particular concentration of the aluminum chloride. The first point and the second point represent the target pattern and the spiral pattern, respectively. The remaining points represent turbulence-like patterns. For the target patterns and the spiral patterns, the spatial means of the temporal standard deviations for the propagating wave velocity angles are low with small spatial standard deviations of the temporal standard deviations. In contrast, the turbulence-like patterns have high spatial means of the temporal standard deviations for the propagating wave velocity angles with large spatial standard deviations of the temporal standard deviations. 

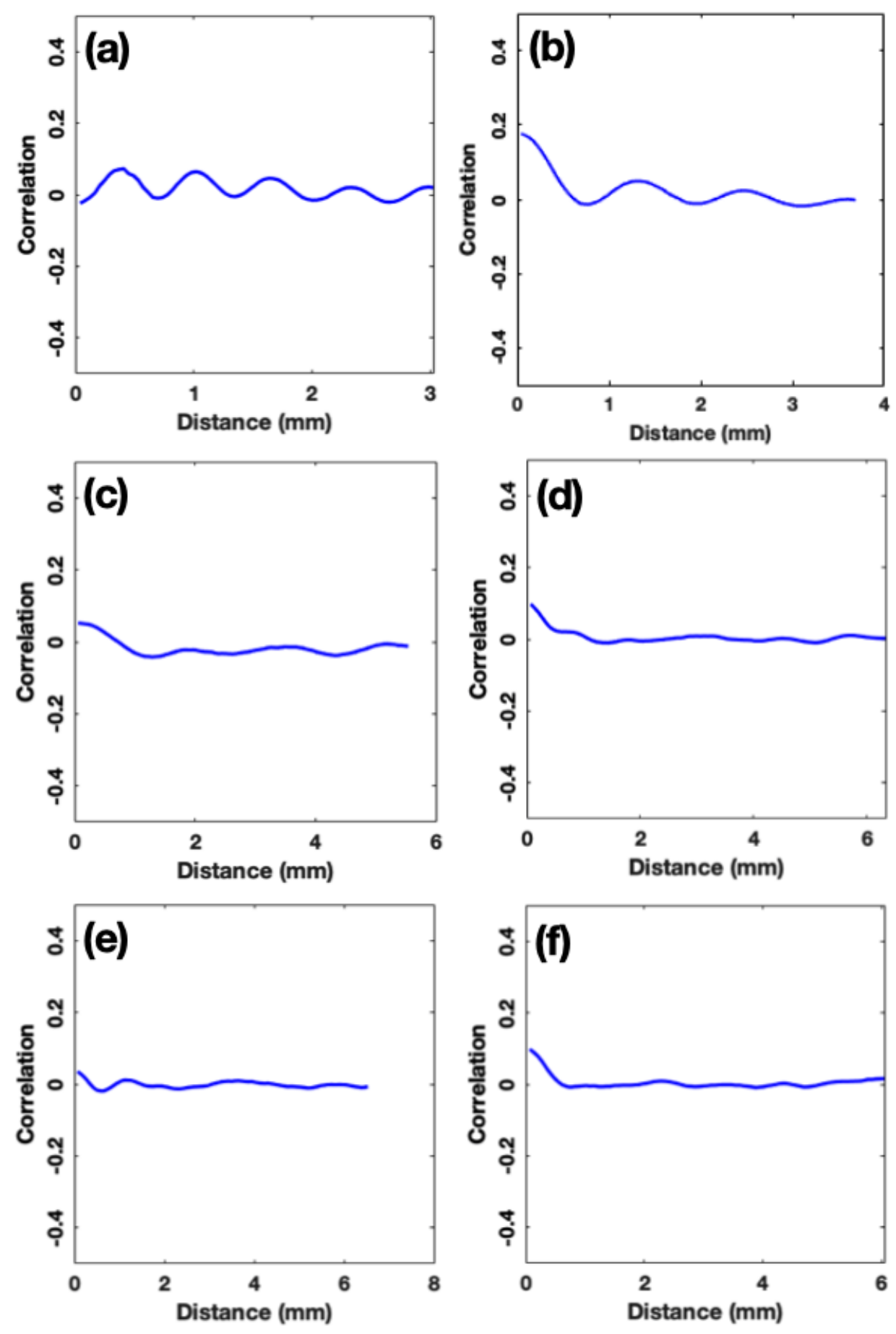

Figure 5.4: Spatial correlation of the patterns formed in the agarose gel at different initial concentrations of the aluminum chloride. $\left[\mathrm{AlCl}_{3}\right]_{0}$ : (a) $0.30 \mathrm{M}$, (b) $0.31 \mathrm{M}$, (c) $0.35 \mathrm{M}$, (d) $0.40 \mathrm{M}$, (e) $0.43 \mathrm{M}$, and (f) $0.45 \mathrm{M}$. 

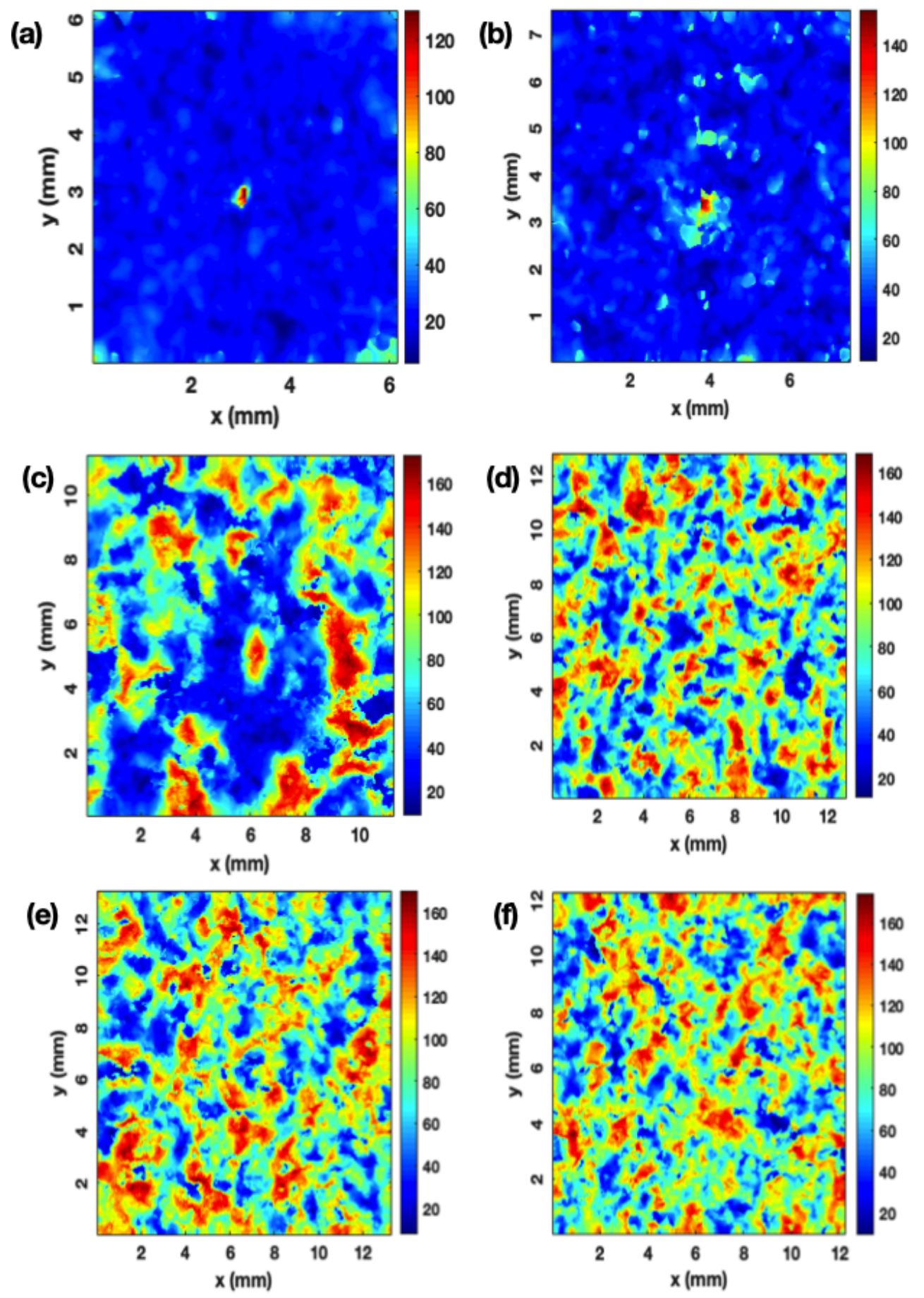

Figure 5.5: Temporal standard deviation of the propagating precipitation wave velocity angles. The colors for each panel correspond to the values of the temporal standard deviation in that region. The initial concentration of the aluminum chloride for each panel is (a) 0.30 $\mathrm{M}$, (b) $0.31 \mathrm{M}$, (c) $0.35 \mathrm{M}$, (d) $0.40 \mathrm{M}$, (e) $0.43 \mathrm{M}$, and (f) $0.45 \mathrm{M}$. 


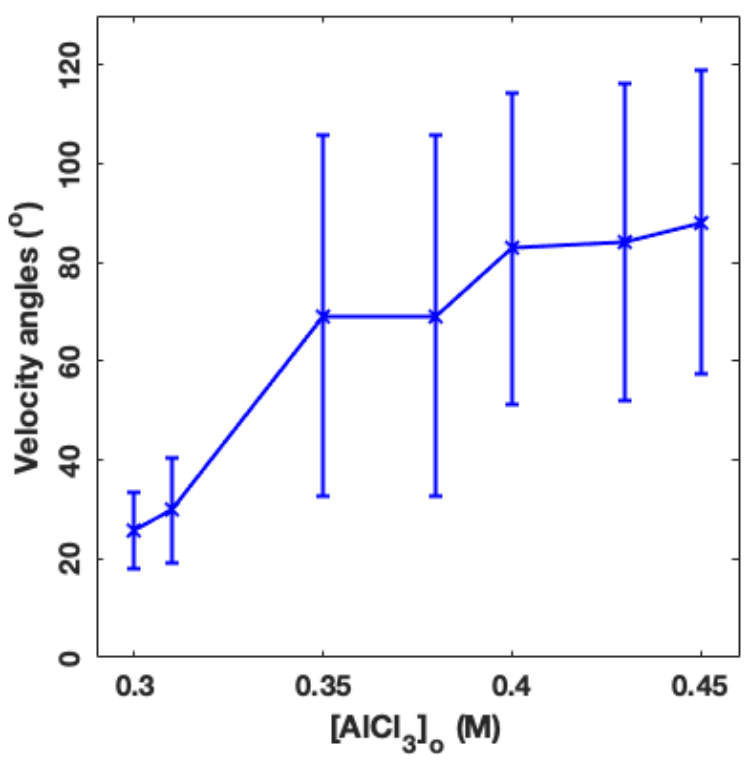

Figure 5.6: Plot of spatial means of temporal standard deviations for velocity angles as a function of initial aluminum chloride concentrations. The error bars represent the spatial standard deviations of the temporal standard deviations for the precipitate velocity angles.

\subsection{Discussion}

The patterns formed in the agarose gel transition from target patterns and spiral patterns to turbulence-like patterns as the initial concentrations of the aluminum chloride increases. This behavior has been observed in past studies of precipitation systems. ${ }^{12}$ The reaction medium is filled with multiple wave centers faster as the initial concentration of the aluminum chloride is increased beyond the transition concentration.

Several techniques are used to characterize the patterns that are observed in this system. One way of characterizing the patterns formed is using the optical flow technique. The optical flow technique allows for the measurement of the moving objects velocity by comparing images taken at different times. $\frac{17}{17}$ In this system, the patterns formed evolve as a function of time. Thus, the optical flow technique allows for the measurement of the propagating wave velocity in this system. Figure 5.2 shows the velocity field of the propagating waves superimposed onto its image. 
At low initial concentrations of the aluminum chloride $(\leqslant 0.31 M)$, the velocity field originates from the center of the reaction medium, which corresponds to the center of the target patterns and the spiral patterns (see Figure $5.2(\mathrm{a})$ and (b)). The velocity field points outward from the target pattern center and the spiral pattern center, which suggest the existence of a source in the propagating waves dynamics.

The existence of a source or a sink in the dynamics can be shown by calculating the divergence of the velocity field of the propagating waves. Figure 5.3(a) and (b) show the results of such calculations for patterns in Figure 5.2(a) and (b), respectively. The center of Figure 5.3(a) and (b) show high positive values, which correspond to a strong source. This means that there is an outflow of propagating waves from the center of the target and spiral patterns.

When the initial concentration of the aluminum chloride is increased to $0.35 \mathrm{M}$, an increase in the number of wave centers is observed. This is evident from the velocity fields and the divergence calculations as shown in Figure 5.3(c). The outward flow of the propagating precipitation waves originated from multiple sources, and these propagating waves propagate and then annihilate each other when the waves collide head on. The head on collision sites act as the sink regions.

The spatial correlation function provides a measure of disorder for a particular pattern. 1 In this study, the spatial correlation function compares the grayscale values of two different regions in the same image. If the two regions are correlated, the spatial correlation value for these two regions will be either positive or negative. This indicates that the grayscale values of these two regions are dependent on each other. However, if the grayscale values of the two regions are uncorrelated, the spatial correlation values will be zero. This indicates that the grayscale values at these two regions are independent of each other.

In the case of target patterns and spiral patterns, there are alternating greyscale 
values between regions with the precipitate presence (dark regions) and regions with the precipitate absence (light regions). Since there are repeating patterns of dark and light regions, the spatial correlation values are expected to be oscillating between positive and negative values. A positive correlation value indicates that the two regions have similar grayscale values (dark regions with dark regions or light regions with light regions). On the other hand, if the spatial correlation value turns out to be negative, the two regions should have two opposing grayscale values (dark regions with light regions). The oscillating spatial correlation values for the target pattern and the spiral pattern are demonstrated in Figure $5.4(\mathrm{a})$ and (b), respectively. For experiments with the initial concentrations of the aluminum chloride at $0.35 M$ or higher, turbulence-like patterns are observed. Therefore, the spatial correlation values are expected to decay to zero, which indicates that there is no correlation between the two regions. Figure 5.4 (c)-(f) show that this is the case.

For target patterns and spiral patterns, the velocity angles are expected to be relatively constant in time since the propagating precipitation waves are expected to propagate in the same direction to maintain the patterns. Thus, the standard deviations of the velocity angles across time are expected to be small, and this is evident from Figure 5.5(a) and (b). The high standard deviation values at the center of the image can be attributed to the center of the target patterns and the spiral patterns. In a properly formed target pattern, such high standard deviations of the velocity angles might not exist. However, in this study, the center of the target patterns is actually a pair of counter-rotating spiral waves. The tip of the waves changes direction as it rotates, and the direction change causes large standard deviations across time. Similar argument can be used to describe the center of the spiral patterns. The tip of the spiral waves continuously changes direction as it rotates, which causes large temporal standard deviations at the center of the image.

In contrast to the target patterns and the spiral patterns, the turbulence-like pat- 
terns are expected to have high standard deviations across time throughout the entire patterns. This is because turbulence-like patterns have several initiation wave centers, and the waves from different centers propagate and collide with each others. The collisions cause some of the waves to be annihilated while some of the waves change their trajectories. The constantly propagating, colliding, annihilating, and changing directions cause the velocity angles of the waves to constantly change as a function of time. Thus, several regions with high temporal standard deviations are produced. This is evident from Figure 5.5(c)-(f).

By calculating the spatial means and the spatial standard deviations of the temporal standard deviations of the velocity angles, these values can be plotted as a function of initial concentrations of the aluminum chloride, and such a plot can be seen in Figure 5.6. With target patterns and spiral patterns, the spatial means and the spatial standard deviations of the temporal standard deviations for the propagating precipitation waves are lower than its turbulence-like patterns counterpart. This reinforces the idea that in target patterns and spiral patterns, the velocity angles do not change significantly in time and space. On the other hand, the spatial means and the spatial standard deviations of the temporal standard deviation for the propagating precipitation waves are higher in turbulence-like patterns, which show that the velocity angles vary significantly both in time and space.

\subsection{Conclusion}

In this study, the patterns in the aluminum chloride/sodium hydroxide system are characterized using the velocity fields, the source or sink identification technique, the spatial correlation function, and the velocity angles statistics of the propagating precipitation waves. Based on the images taken from the experiments, target patterns and spiral patterns form at low initial concentrations of the aluminum chloride $(\leqslant 0.31 M)$, while turbulence-like 
patterns form at higher concentrations of the aluminum chloride $(\geqslant 0.35 M)$.

Several techniques are employed to characterize the dynamics of the system. The velocity fields, which are determined using the optical flow technique, show that the propagating precipitation waves originate from the center of the target patterns and the spiral patterns. These waves propagate outward from the center of the patterns. There are typically a single origin of the precipitation waves at a given area at low initial concentrations of the aluminum chloride. The source or sink identification technique results in Figure 5.3 show that the centers of the target patterns and the spiral patterns act as the source of the propagating precipitation waves. More wave centers appear at higher initial concentrations of the aluminum chloride, and this translates to a higher number of sources and sinks in the velocity fields.

The spatial correlation function is also used to characterize the patterns formed as a function of the initial concentrations of the aluminum chloride. At low concentrations of the aluminum chloride, the target patterns and the spiral patterns formed result in the oscillating spatial correlation values. The oscillating spatial correlation values can be attributed to the alternating patterns within the target patterns and the spiral patterns. At higher initial concentrations of the aluminum chloride, the spatial correlation values are observed to decay to zero. This indicates that after some distance, the regions in the image are uncorrelated. This behavior is attributed to the uncorrelated regions as a result of turbulence-like patterns.

Basic statistics of the velocity angles are used to describe the patterns formed in this system. The temporal standard deviations of the precipitate velocity angles are calculated. Figure 5.5 shows the results for such calculations. Target patterns and spiral patterns show low temporal standard deviations everywhere except at the center of the target patterns and the spiral patterns. In contrast, turbulence-like patterns show high temporal standard deviations at multiple regions within the reaction media. 
The spatial means and the spatial standard deviations of the temporal standard deviations are calculated and plotted as a function of the aluminum chloride initial concentrations in Figure 5.6. Target patterns and spiral patterns are shown to have low spatial means of the temporal standard deviations with small spatial standard deviations of the temporal standard deviations with the exception at the center of the patterns. On the other hand, turbulence-like patterns have high spatial means of the temporal standard deviations with large spatial standard deviations of the temporal standard deviations. 


\subsection{References}

[1] J. Maselko and K. Showalter, Phys. D 49, 21 (1991).

[2] F. Fenton, E. M. Cherry, H. M. Hastings, and S. J. Evans, Chaos 12, 852 (2002).

[3] A. Murthy, E. Bartocci, F. H. Fenton, J. Glimm, R. A. Gray, E. M. Cherry, S. A. Smolka, and R. Grosu, IEEE/ACM Trans. Comput. Biol. Bioinf. 10, 323 (2013).

[4] G. Ertl, Science 254, 1750 (1991).

[5] R. Field, E. Körös, and R. Noyes, J. Am. Chem. Soc. 94, 8649 (1972).

[6] R. M. Noyes, R. J. Field, and E. Körös, J. Am. Chem. Soc. 94, 1394 (1971).

[7] G. Biosa, S. Bastianoni, and M. Rustici, Chem. - Eur. J. 12, 3430 (2006).

[8] A. N. Zaikin and A. M. Zhabotinsky, Nature 225, 535 (1970).

[9] A. Winfree and W. Jahnke, J. Phys. Chem. 93, 2823 (1989).

[10] Q. Ouyang, H. L. Swinney, and G. Li, Phys. Rev. Lett. 84, 1047 (2000).

[11] L. Q. Zhou and Q. Ouyang, J. Phys. Chem. A 105, 112 (2001).

[12] A. Volford, F. Izsák, M. Ripszám, and I. Lagzi, Langmuir 23, 961 (2007).

[13] M. Tinsley, D. Collison, and K. Showalter, Chaos 25, 064306 (2015).

[14] M. M. Ayass, I. Lagzi, and M. Al-Ghoul, Phys. Chem. Chem. Phys. 16, 24656 (2014).

[15] M. M. Ayass, M. Al-Ghoul, and I. Lagzi, J. Phys. Chem. A 118, 11678 (2014).

[16] K. Y. Wong and C. L. Yip, Pattern Recognition 42, 1371 (2009).

[17] R. G. Townsend and P. Gong, PLoS Comput. Biol. 14, 1 (2018). 